WALDEN

UNIVERSITY

A higher degree. A higher purpose.

Walden University

ScholarWorks

Walden Dissertations and Doctoral Studies

Walden Dissertations and Doctoral Studies

Collection

2018

\title{
Virtual Leadership in Complex Multiorganizational Research and Development Programs
}

Gariann Marie Gelston

Walden University

Follow this and additional works at: https://scholarworks.waldenu.edu/dissertations

Part of the Organizational Behavior and Theory Commons

This Dissertation is brought to you for free and open access by the Walden Dissertations and Doctoral Studies Collection at ScholarWorks. It has been accepted for inclusion in Walden Dissertations and Doctoral Studies by an authorized administrator of ScholarWorks. For more information, please contact ScholarWorks@waldenu.edu. 


\title{
Walden University
}

\author{
College of Management and Technology
}

This is to certify that the doctoral dissertation by

\author{
Gariann Marie Gelston
}

has been found to be complete and satisfactory in all respects, and that any and all revisions required by the review committee have been made.

\section{Review Committee}

Dr. Carol Wells, Committee Chairperson, Applied Management and Decision Sciences Faculty

Dr. Dean Frost, Committee Member, Applied Management and Decision Sciences Faculty

Dr. Howard Schechter, University Reviewer Applied Management and Decision Sciences Faculty

Chief Academic Officer

Eric Riedel, Ph.D.

Walden University

2018 


\begin{abstract}
Virtual Leadership in Complex Multiorganizational Research and Development Programs by

Gariann Marie Gelston
\end{abstract}

MEM, Washington State University, 1998

BS, Colorado Mesa University, 1991

Dissertation Submitted in Partial Fulfillment
of the Requirements for the Degree of
Doctor of Philosophy
Applied Management and Decision Sciences

Walden University

May 2018 


\begin{abstract}
A 2002 congressional mandate initiated the U.S. Department of Homeland Security's (DHS) Centers of Excellence programs with a requirement to conduct crossorganizational research and development. The resulting complex multiorganizational programs required more effective virtual leadership and management strategies. Fifteen years later, the presidential budget showed that $61 \%$ of the DHS budget was targeted for such research and development. The complex management strategies and virtual leadership skills required to lead the programs were lacking, as top scientific researchers are drawn upon to manage programs. The purpose of this study was to understand followers' perspectives regarding virtual leadership and collaboration within complex multiorganizational DHS Centers of Excellence programs. Complex-systems and leadermember exchange theories formed the conceptual framework. Fifteen individuals, representing 10 Centers of Excellence programs, were interviewed about virtual leadership strategies used to motivate highly educated scientists across program organizations. A case study analysis of participants' perspectives revealed 4 key findings. The first finding was that programs employed shared leadership where project subteams were self-managed. The second finding was that the programs focused on applied research, resulting in subteam structures segmented by discipline. The third finding showed that collaboration occurred within collocated subteams and coordination was most common between virtual partners. The final finding was that highly educated participants were primarily self-motivated. Targeted training can lead to positive social change through influencing the existing paradigm of leadership for these programs.
\end{abstract}


Virtual Leadership in Complex Multiorganizational Research and Development Programs by

\author{
Gariann Marie Gelston
}

MEM, Washington State University, 1998

BS, Colorado Mesa University, 1991

Dissertation Submitted in Partial Fulfillment
of the Requirements for the Degree of
Doctor of Philosophy
Applied Management and Decision Sciences

Walden University

May 2018 


\section{Dedication}

I would like to dedicate this work to God and my loving family. I owe much to my husband who encouraged me to start this journey and has been by my side throughout. I am thankful for the patience and support of my children Megan, Suzi, Katie, and Andy who have grown up in an atmosphere of inquiry and exploration; and who share my love of learning. My family has given up significant time and attention to this effort. I appreciate all of their love and sacrifice. 


\section{Acknowledgments}

I would like to acknowledge Dr. Wells for her unwavering support of my research. She provided guidance, encouragement, and commitment to this work without which I would not have been able to complete my research. Dr. Wells provided incredible mentoring and inspiration during times of defeat.

I would like to thank Pacific Northwest National Laboratory for their support for this program, allowing me to spend the time to work through the processes, and commitments, of completing a $\mathrm{PhD}$ program while working full time. I would specifically like to thank my manager Diana Shankle for providing mentoring support throughout the program.

I would like to acknowledge the many people who helped in my journey of writing through formal, or informal, contributions. My friend Baraba Reichmuth, who took the time to read my material, and share her thoughts; and my friend Dr. Angela Dalton who sat with me for many lunch hours brainstorming methods, interview questions, and organization ideas. I would like to acknowledge Cornelia Brim and Jessica Cole, my friends and reviewers, who provided insights into my writing. Finally, I'd like to thank my daughter Suzanne, who patiently read through many chapters of my final report to assist in producing a quality product. 


\section{Table of Contents}

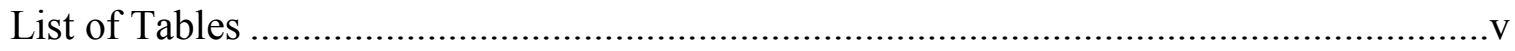

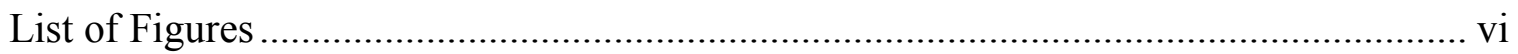

Chapter 1: Introduction to the Study .......................................................................

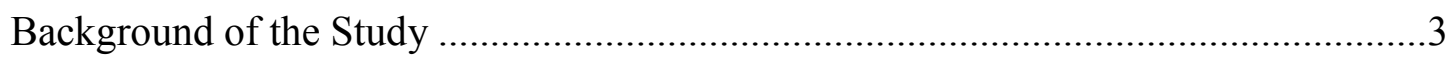

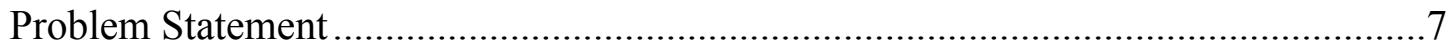

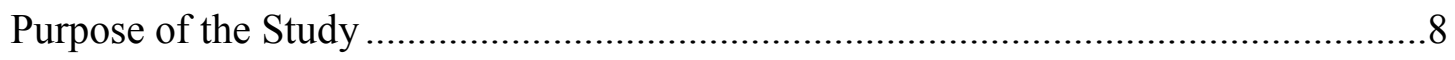

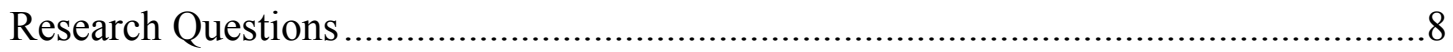

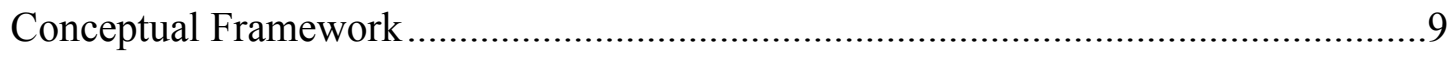

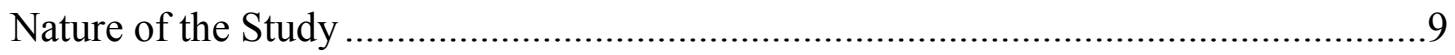

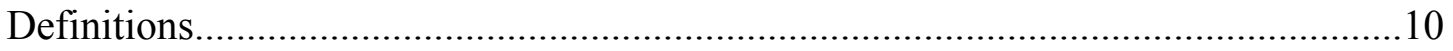

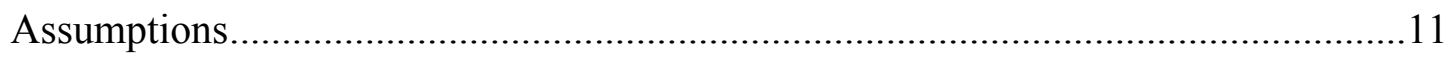

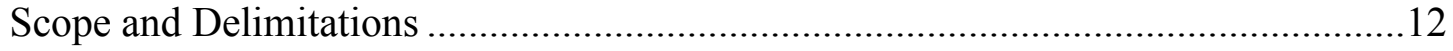

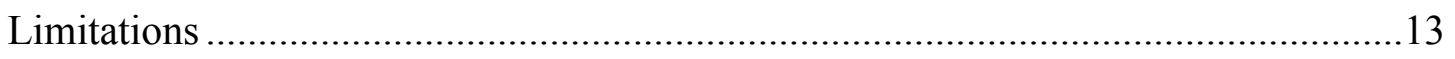

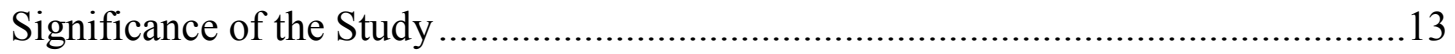

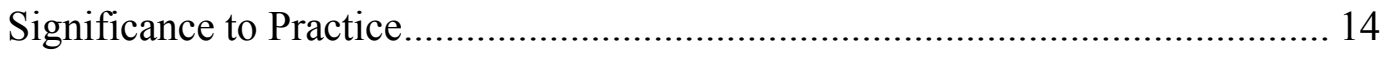

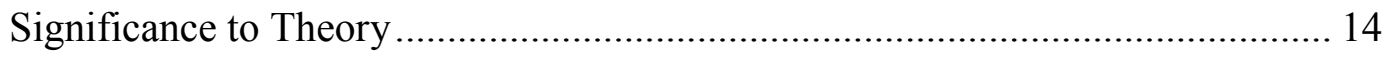

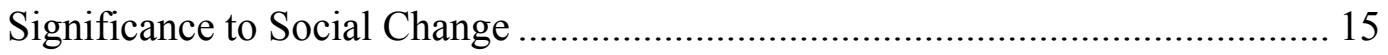

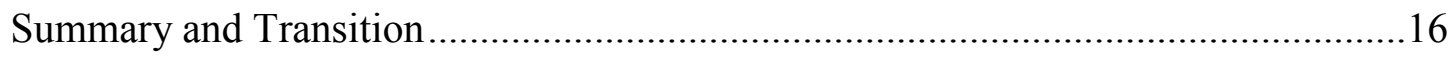

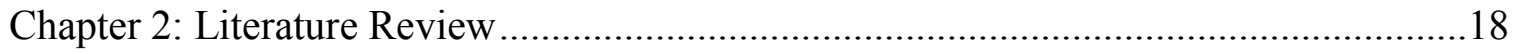

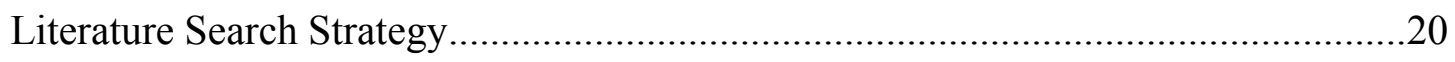

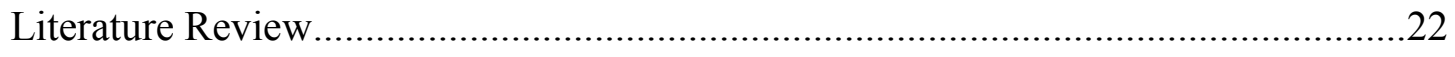




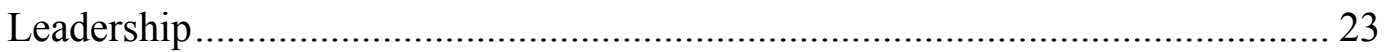

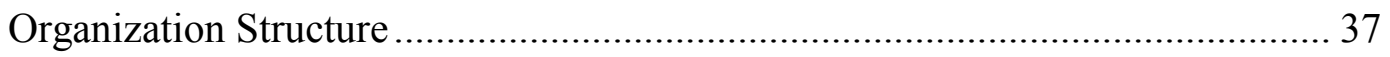

Scientific Collaboration Environments ............................................................ 43

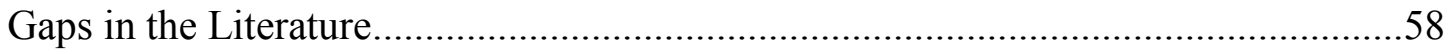

Gap 1: Holistic Exploration of Leadership ……………………….................... 58

Gap 2: Multiorganizational Constructs in Scientific Collaborations..................... 61

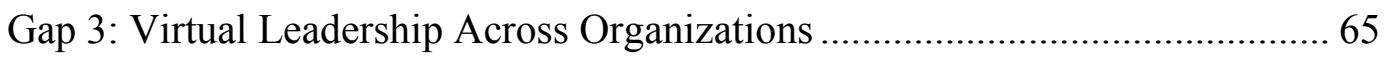

Gap 4: Scientific Collaboration Environments ………….................................... 70

Gap 5: Lack of Leadership Training ............................................................... 73

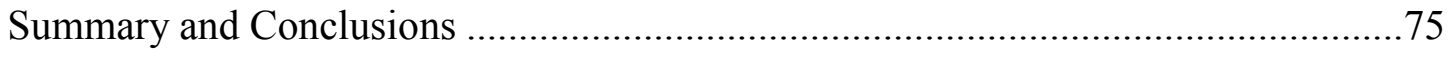

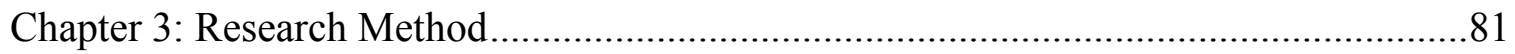

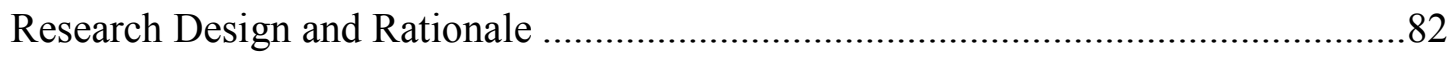

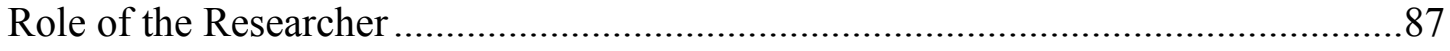

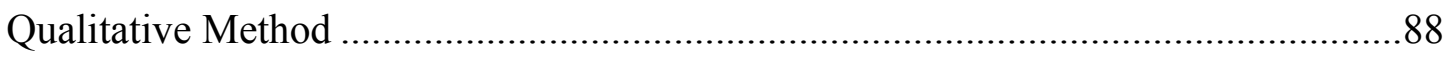

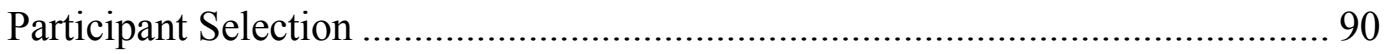

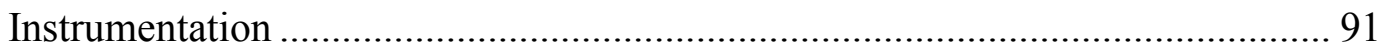

Procedures for Recruitment, Participation, and Data Collection........................... 93

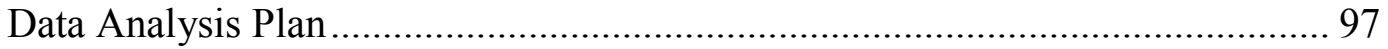

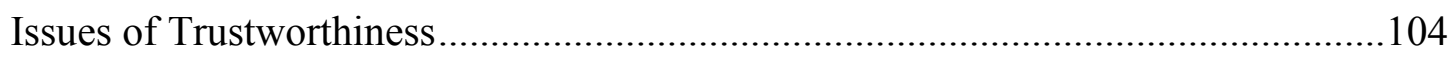

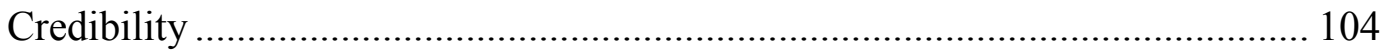

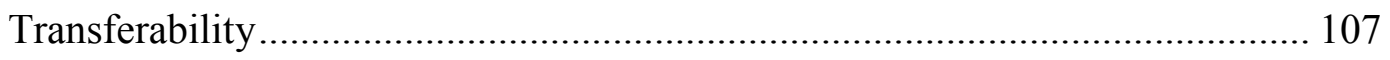

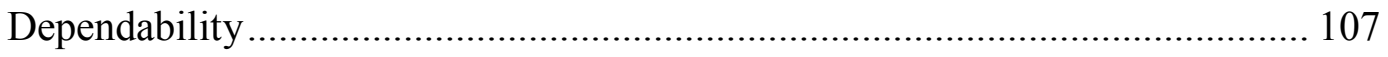




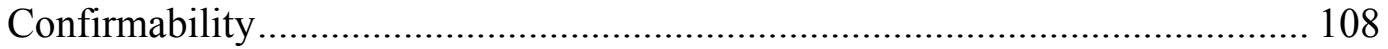

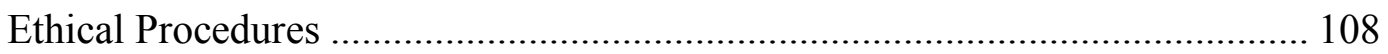

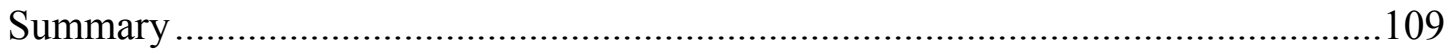

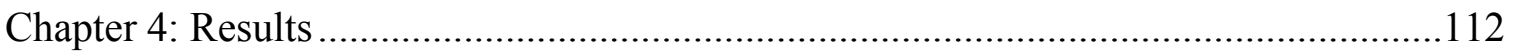

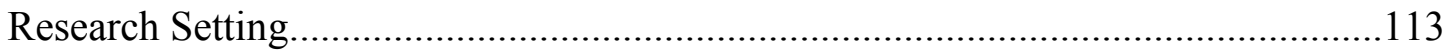

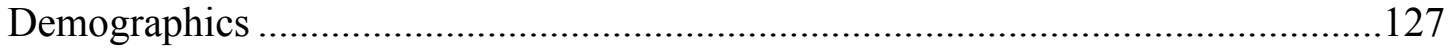

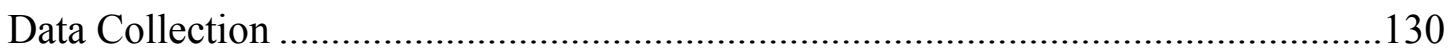

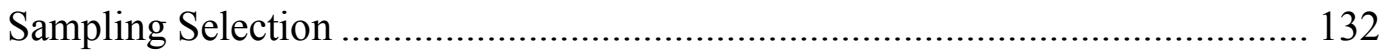

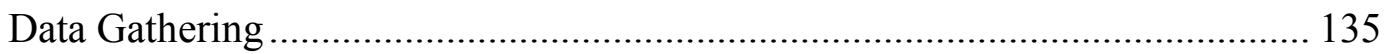

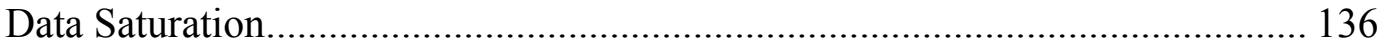

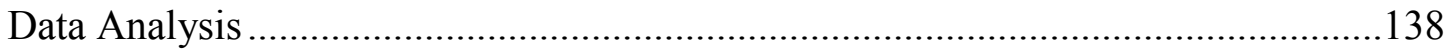

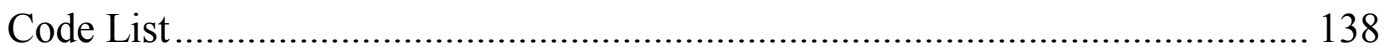

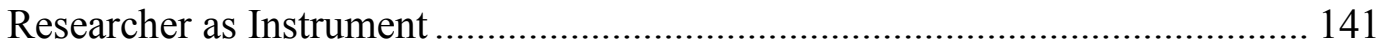

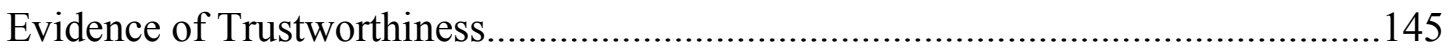

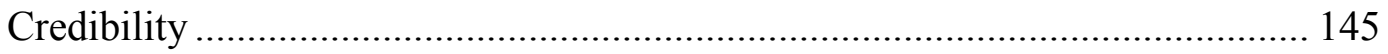

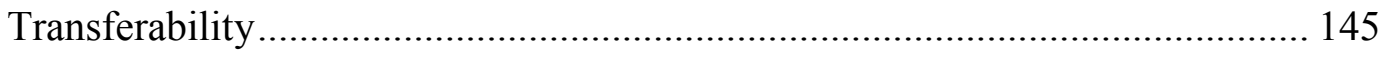

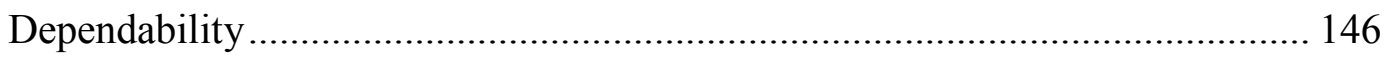

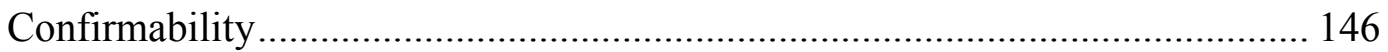

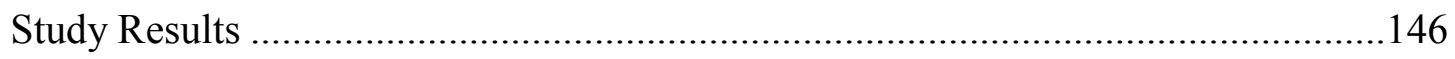

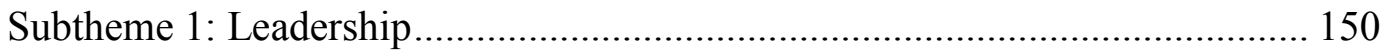

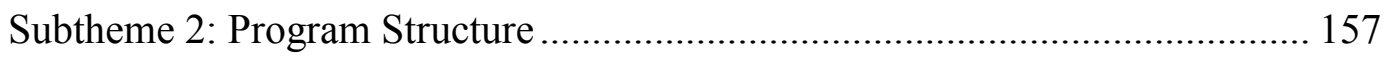

Subtheme 3: Virtual Environment ................................................................. 168 
Subtheme 4: Research Culture

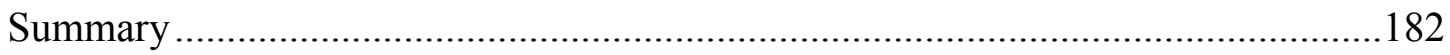

Chapter 5: Discussion, Conclusions, and Recommendations .....................................186

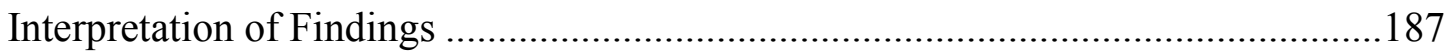

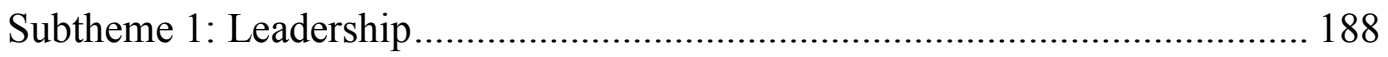

Subtheme 2: Program Structure ................................................................. 191

Subtheme 3: Virtual Environment ............................................................. 197

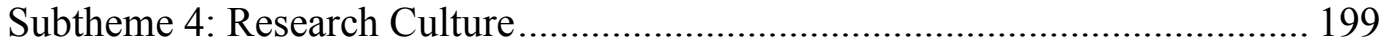

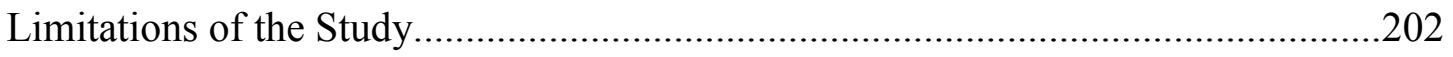

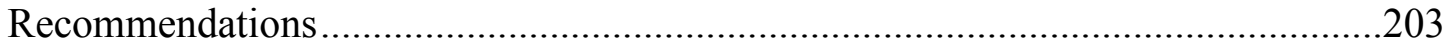

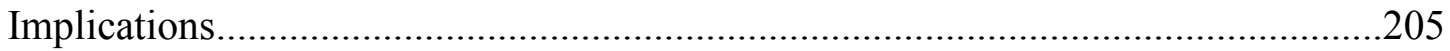

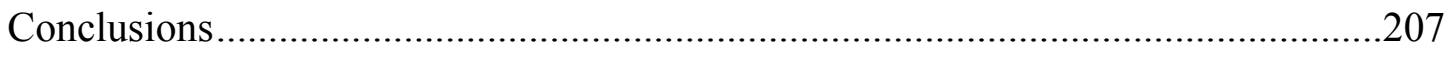

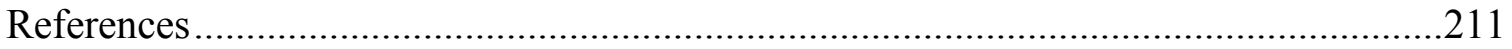

Appendix A: Telephone Interview Protocol ......................................................233

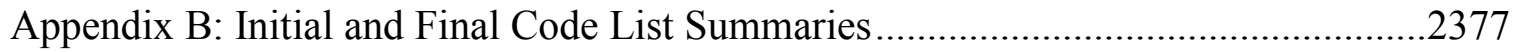




\section{List of Tables}

Table 1. New 2017 DHS Centers of Excellence Programs and Focus Areas ................ 115

Table 2. Current DHS Centers of Excellence Program Focus Areas ......................... 116

Table 3. Emeritus DHS Centers of Excellence Program Focus Areas ......................... 117

Table 4. Former DHS Centers of Excellence Program Focus Areas ............................ 118 


\section{List of Figures}

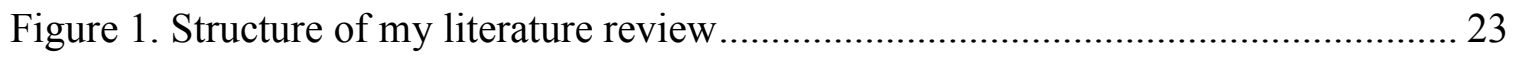

Figure 2. Package of evidence for each interview participant .......................................... 96

Figure 3. Code development and analysis process …………………......................... 105

Figure 4. Number of organizations within each program ........................................... 121

Figure 5. Number of years the program was active ................................................... 123

Figure 6. Number of publications listed within each program .................................... 124

Figure 7 Number of participants identified for each program ..................................... 126

Figure 8. Hierarchy of the DHS Centers of Excellence programs................................ 128

Figure 9. Years of experience of each participant interviewed .................................... 129

Figure 10. Data collection and analysis preparation process ........................................ 131

Figure 11. Summary of participation and responses tracking...................................... 133

Figure 12. Process code list by participant .............................................................. 143

Figure 13. Descriptive code list by participant ............................................................. 144

Figure 14. Four subthemes of research question. .................................................... 146

Figure 15. Number of organizations represented by the participants interviewed. ........ 148

Figure 16. Number of operational years $t$ represented by the participants interviewed .149

Figure 17. Exploratory concepts within the leadership subthem.................................. 151

Figure 18. Exploratory concepts within the program structure subtheme ...................... 160

Figure 19. Exploratory concepts within the virtual environment subtheme................... 169

Figure 20. Exploratory concepts within the Research culture concepts ......................... 176

Figure 21. General program structure …………………........................................ 194 
Figure 22. Proposed research engagement framework ............................................. 195 
Chapter 1: Introduction to the Study

Leadership for complex interdisciplinary research and development programs is critical to global and national security; however, there has been no documented basis for leadership criteria, nor training requirements for virtual leadership of these crosscutting multiorganizational entities (Chompalov, 2014; Vessey, Barrett, Mumford, Johnson, \& Litwiller, 2014). The purpose of this qualitative case study was to understand followers' perspectives regarding virtual leadership within complex interdisciplinary multiorganizational programs. For this case study, the participant pool was members of U.S. Department of Homeland Security (DHS) Centers of Excellence programs. The DHS Centers of Excellence program charter was designed to commission universities and coordinated organizations to collectively apply novel thinking to the nation's problems related to national security. The choice of universities as leaders was to fulfill the goal to help train the next generation of homeland security experts. The DHS Centers of Excellence programs worked closely with academia, industry, first responders, and DHS operational components such as Coastguard, Customs and Border Protection, and the Transportation Security Administration (U.S. DHS, 2017). These programs were expected to develop customer-driven, innovative tools and technologies to solve realworld challenges. Each program targeted national security technical areas focused on specific real-world challenges. In this way, programs were designed to bridge academic environments and applied settings. This bridging expectation in itself adds to the complexities faced by DHS Centers of Excellence leaders. 
The intersection of institutionally complex programs (Jay, 2013) and highly educated participants (Paulsen, Callan, Ayoko, \& Saunders, 2013) yields a new area of organizational leadership research not addressed in the literature. The present research provides a unique member perspective regarding virtual leadership strategies for fostering creativity, crossing organizational boundaries, encouraging interdisciplinary collaboration, and providing customer-driven solutions.

The research and development programs I discuss in this dissertation were composed of multiple suborganizations of scientists coalescing into a temporarily unified organization. For the DHS Centers of Excellence programs, one university generally serves as the primary owner of the program, with other universities, industries, and national laboratory partners collectively participating in research (U.S. DHS, 2017). The interdisciplinary nature of these multiorganizational programs contributes to the complexity of the functional interactions among individuals involved. The virtual nature of these programs arose from the need to draw on experts wherever they were located. Bringing together experts from across the country necessitates addressing the virtuality of the program's organization.

Typically, government program managers select program leaders from established researchers in relevant fields. The interdisciplinary challenge was to maintain a diverse team of research expertise. The elements brought together were a virtual, complex, multiorganizational, and research and development environments with leadership challenges. 
In this chapter, I describe the purpose of my study with a summary of related literature through a discussion of the background. I also provide the basis for the research question by stating the management problem addressed by my study. A single research question was considered to support the conceptual framework that served as the foundation for the research approach. I describe the definitions and assumptions to provide an understanding of the scope, as well as limitations, of this research. Finally, I highlight the significance of the study as it relates to management practice, management theory, and social change.

\section{Background of the Study}

In 2002, a congressional mandate introduced the idea of DHS Centers of Excellence, which encouraged crossorganizational research and development teams (U. S. DHS, 2017). Wanting to draw together top researchers across organizations to address the change and increasingly complex national security challenges, the U.S. Congress drafted the Homeland Security Act in 2002.

The Secretary, acting through the Under Secretary for Science and Technology, shall establish . . . a university-based center or centers for homeland security. The purpose of this center or centers shall be to establish a coordinated, universitybased system to enhance the nation's homeland security. (Homeland Security Act, 2002)

In 2010, the U.S. Department of Energy (DOE) expressed a similar interested in multiorganizational programs when they introduced the concept of innovation hubs. These hubs were interdisciplinary teams drawn from multiple organizations brought 
together to collaborate (Cho, 2013; DOE, 2014; Moniz, 2012). The National Science Foundation (2018) sponsored similar science and technology centers. These interdisciplinary program environments were multiorganizational and usually disbursed geographically, which results in complex organizational settings.

In 2004, the DHS began the Centers of Excellence programs process by selecting participants for an extended consortium of research and development programs targeted at particular homeland security challenges. These programs included universities, industries, and national laboratories working cooperatively. Although there were significant financial investments in these programs, not all program leaders were able to establish sustainable constructs for meaningful solutions (Bozeman, Fay, \& Slade, 2013; $\mathrm{Su}, 2014)$. Researchers have studied various individual aspects of virtual leadership, interdisciplinary teams, complex organizations, and scientific collaborations. Combining these concepts may provide insight on the best way to explore programs (Hazy \& UhlBien, 2015; Henry, 2015).

Leadership and organizational theorists have begun to consider the aspect of leadership in complex systems (Hazy \& Uhl-Bien, 2015; Long, Cunningham, Wiley, Carswell, \& Braithwaite, 2013). For example, Jay (2013) provided a perspective on leadership that included a consideration of the complexity of hybrid organizations - those entities brought together to spur innovation. More recently, Henry (2015) extended the concept of hybrid organizations to more complex multiorganizational systems. Murase, Carter, DeChurch, and Marks (2014) focused their research on the challenges of multiple team systems and looked at the focus, function, and forms of leadership. Wageman and 
Fisher (2014) discussed the concept of leadership of complex organizational systems targeting the idea of legitimate authority. Wageman and Fisher addressed options that included centralized leadership versus multilevel vertical leadership. Four areas of legitimate authority put forth by Wageman and Fisher were (a) executing tasks, (b) monitoring and managing, (c) designing the team, and (d) guiding overall direction.

Previous research has not addressed complex organizational systems that require virtual leadership. Several researchers suggested a need for new ways to look at the challenges of virtual leadership by considering them as complex organizational systems (Cady, 2016; Collinson, 2014; Hazy \& Uhl-Bien, 2015; Luciano, DeChurch, \& Mathieu, 2015). Luciano et al. (2015) noted the need for new metrics of leadership in multiple team programs. Hazy and Uhl-Bien (2015) described the leadership of these systems as a new area that needs to be explored further from a leadership perspective. Collinson (2014) suggested considering new angles from which to conduct leadership research. Collison's suggestions allowed scholars to understand the leadership constructs and reconsider the dichotomies that often surface in leadership theory. Cady (2016) used a system-of-systems lens to aid in understanding the challenges of complex leadership.

Theorists addressed the nuances associated with highly educated participants in research communities (Vessey et al., 2014; Walsh \& Huang, 2014). Vessey et al. (2014) described the leadership of scientists as involving the challenge of leading creative individuals. Other researchers recommended additional research on the leadership of complex organizational systems. The existing studies on the leadership of complex organizational systems included topics on scientific prestige and culture (Walsh \& 
Huang, 2014), trust building (McNab, Basoglu, Sarker, \& Yu, 2012), technology (BoschSijtsema, Fruchter, Vartianinen, \& Ruohomaki, 2011), and politics (Ellen, 2014). According to Shrum, Genuth, and Chompalov (2007), several aspects of scientific collaboration required analysis including the scale, organizational structure, technology, and information interdependencies. Shrum et al. described relationship characteristics that were also important to scientific collaboration, including trust, conflict, and performance.

The interdisciplinary nature of scientific collaborations was a challenge faced by leaders of research and development programs. Kuhn (2012) noted that early scientific thinkers were often very close friends with scientists of multiple disciplines. He discussed the separation, or silos, which appeared when universities came into existence, and the resulting academic departments became competitors rather than collaborators. Over time, the ability to communicate among disciplines declined, and professional incentives promoted a competitive environment when organizations began to compete for research and funding (Kuhn, 2012). Years of competing for research funding has resulted in organizations striving to distinguish themselves from each other (Sanberg et al., 2014; Walsh \& Huang, 2014). These disciplinary silos still exist today. In addition to the competition, geographic separation makes communication between frequently competing researchers more difficult. The virtual leadership of these complex organizational systems becomes critical as federally funded more consortiums of research and development organizations are established to bring together multiple disciplines (Sidhu \& Volberda, 2011). 
The first DHS Centers of Excellence programs have reached the end of their initial 12-year life-cycle. Some continue to receive support; others have been cut short and moved into an emeritus or former status (U.S. DHS, 2017). Recent studies have addressed the challenges of program management across multiple organizations. Program success has focused on different management structures (Henry, 2015; Manning \& Roessler, 2014; Turkulainen, Ruuska, Brady, \& Artto, 2015). Leader-member relationships require additional focus to improve our understanding of the strategies employed by virtual leaders when operating in a virtual crossorganization collaborative environment.

\section{Problem Statement}

The lack of understanding of leadership expectations in complex multiple organizational models poses a management research challenge (Hoegl \& Muethel, 2016; Sahay \& Baul, 2014). The general management problem was that no management models existed for leadership in virtual interdisciplinary scientific collaborations. It was important to gain an improved understanding of leadership, as it relates to management when considering leaders for virtual complex organizational structures (Jay, 2013; Matzler, Strobl, \& Bailom, 2016). For example, virtual team leadership has required 50\% more time than traditional collocated teams because of the increased burden of relationship building in geographically dispersed teams (Dyer, Dyer, \& Dyer, 2013). Scientists-turned leaders of complex collaborations are not traditionally trained in complex multiorganizational management, and may not have the necessary experience to lead virtual interdisciplinary collaborations (Leiserson \& McVinney, 2015). The specific 
management problem was that for the DHS Centers of Excellence programs, the participants' expectations of leaders were not well understood. Research in this area could provide insights into future leadership selection criteria. Research on leadership has mainly addressed leadership traits and their relationship to organizational outcomes (Hazy \& Uhl-Bien, 2015; Henry, 2015). Only a few researchers have focused on developing methods for leadership evaluation that they based on organizational characteristics and contexts (Vessey et al., 2014) such as virtual organizations, interdisciplinary teams, and multiorganizational collaborations.

\section{Purpose of the Study}

The purpose of this qualitative case study was to understand followers' perspectives regarding virtual leadership and collaboration within complex multiorganizational DHS Centers of Excellence programs. I characterized successful leadership of complex multiorganizational research and development programs as fostering creativity, crossing organizational boundaries, encouraging interdisciplinary collaboration, and providing customer-driven solutions (U.S. DHS, 2017). The investigation into virtual leadership strategies in these complex interdisciplinary systems provided insight into the development of methods for program design, management, and leadership theory.

\section{Research Questions}

In this study, I proposed a single overarching research question: What virtual leadership strategies do leaders of interdisciplinary collaborations use to motivate their highly educated scientists across organizations? This question guided my exploration into 
the challenges faced by scientists-turned virtual leaders of complex multiorganizational interdisciplinary research and development programs. To further inquire about specific elements of my research question, I broke down the question into four subthemes: leadership, program structure, virtual environment, and research culture. I examined leadership of these complex scientific collaborations from the followers' perspectives, which offered a lens through which to enhance understanding of virtual leadership.

\section{Conceptual Framework}

The conceptual framework for this study was drawn from emerging research regarding leader-member exchange theory (Schermuly, Mayer, \& Dämmer, 2015) and complex-systems theory (Cady, 2016; Hazy \& Uhl-Bien, 2015; Long et al., 2013). These theories, along with research on virtual leadership (Hoch \& Kozlowski, 2014) and research on interdisciplinary scientific collaboration environment (Bozeman et al., 2013; Lariviere, Gingras, Sugimoto, \& Tsou, 2015), provided the conceptual framework for this study. I looked at the virtual leader-member relationships from the members' perspectives. These emerging leadership structures for complex-systems were combined with scientific collaboration factors to provide a structure for the conceptual framework for this study. The goal of understanding virtual leadership strategies in interdisciplinary scientific collaborations from followers' perspectives guided the research question of this study.

\section{Nature of the Study}

The nature of this study was a qualitative case study design. This design was intended to address the gap in extant research on virtual leadership in multiorganizational 
scientific collaborative environments. Previous researchers called for a more holistic consideration of leadership of complex multiorganizational systems (Dinh et al., 2014; Henry, 2015). Additionally, understanding the context of a program's team dynamics in interdisciplinary collaborations of highly educated individuals was necessary (Vessey et al., 2014). Several qualitative research designs were appropriate for this study, including case study, phenomenology, grounded theory, and ethnography. A case study is an option for complex open learning (Yin, 2014).

I selected a case study design to provide an opportunity for open investigation of multiorganizational research and development programs as complex integrated systems. Using this qualitative case study design, I investigated multiple different, yet parallel, scientific collaboration programs. Few researchers have focused on virtual leaders and their relationships with team members in interdisciplinary scientific collaborations within the context of complex multiorganizational programs. I focused on the scientific collaboration program members' perspectives to allow for an open investigation of program virtual leadership strategies for fostering creativity, crossing organizational boundaries, encouraging interdisciplinary collaboration, and providing customer-driven solutions.

\section{Definitions}

To help clarify the purpose of this study, I use the following definitions.

Collaboration: A "social process whereby human beings pool their human capital for the objective of producing knowledge" (Bozeman et al., 2013, p. 3). 
Multiteam system: Connectivity of the team as a collective unit, with each team contributing unique skills to obtain goals that could not be accomplished independently (Carter \& DeChurch, 2014).

Leader-member exchange: A single level of analysis conducted collectively across three leadership domains; (a) leader, (b) follower, and (c) relationship (Erdogan \& Bauer, 2014). For this discussion, I use the term follower, participant, and member interchangeably.

Interdisciplinary collaboration: The cooperative work that results when researchers reach beyond their disciplines for expertise and operate as a collective with a shared objective (Shrum, Genuth, \& Chompalov, 2007).

Program: “A group of related projects, subprograms, and program activities managed in a coordinated way to obtain a benefit not available from managing them individually" (Project Management Institute, 2013, p. 6).

\section{Assumptions}

Documenting my research assumptions helped provide boundaries for the appropriate use of the data collected and the analysis approach. For this research, interdisciplinary research and development programs function as single complex scientific collaboration programs with an organizational structure providing bounding parameters that were identifiable. In the instance where the participating organizations change over time, the full lifetime of the program from initiation to 2017 served as the bounding criteria for an identifiable set of participants. 
Leadership has multiple possible definitions. Virtual leadership continues to be an emerging field with little previous research to draw on. As part of my research documentation, I discuss insights drawn from a set of participant interviews, and report findings in the Results section (Chapter 4). My research premise assumed that participant interviews would provide insights into virtual leadership. I also assumed that the participant pool was representative of programs conducting scientific collaborations. These assumptions were confirmed during the data collection and analysis phases of my research.

Finally, there was an assumption in my study that research and development organizations selected were predominately highly educated, scientifically-based individuals participating in the crossorganizational interdisciplinary collaboration. The educational underpinning of individuals interviewed was included in my research. The advanced education of individuals participating in multiorganizational research and development scientific collaboration programs was a basis used for additional bounding of the scope of this research.

\section{Scope and Delimitations}

The scope of this study was limited to government-funded research and development programs, as distinguished from commercial-product-centered multiorganizational teams or medical research collaborative teams. This restriction supported the possibility that funding types, source, and stability may change the research results. The scope of my study was focused on research and development programs that include interdisciplinary teams drawn from multiple organizations. These collaborative 
teams included the individual members who do not collocate and have advanced degrees that engender a scientific culture and required some form of virtual leadership. The study included programs with a variety of organizations; this environment supported the investigation into the challenges of virtual leadership across disciplinary, geographical, and organizational differences.

\section{Limitations}

Limitations of this study were the amount and kind of information inferred from the interviews conducted regarding virtual leadership and leader-member relationships perceptions. There was limited extensibility of the findings because the information gathered was specific to a targeted set of DHS programs. The findings require additional research through statistically defensible approaches to gain general insights applicable to broader populations.

\section{Significance of the Study}

Ideally, this study illuminates the intersection of institutionally complex programs, and the influence of highly educated participant research (Dinh et al., 2014; Henry, 2015) on the resulting programs that operate in an interdisciplinary virtual environment (Hoegl \& Muethel, 2016). This environment presented a new area of organizational leadership research. My study was focuses on the exploration of virtual leadership strategies within those programs. The results provided member-based insight on leadership strategies. The strategy knowledge supports the development of virtual leadership training and methods to the successful assembly of interdisciplinary project teams. Actionable information provided to program owners may result in enhanced 
leadership effectiveness in these interdisciplinary scientific collaborations. In turn, increased leadership effectiveness could result in groundbreaking teams needed to solve challenges in energy, water resource management, climate change, and national security fields. Over the longer term, this study could be the basis for enhanced leadership education for scientists. It provides a unique member perspective regarding DHS (2017) program goals for fostering creativity, crossing organizational boundaries, encouraging interdisciplinary collaboration, and providing customer-driven solutions.

\section{Significance to Practice}

Investigation of the virtual leadership of multiorganizational research and development programs could influence positive social change in practice through informing leadership models. My study diverged from addressing leadership traits and how traits relate to organizational outcomes. I brought insights into organizational research to include characteristics and contexts to provide alternative views of organizational leadership research. Additional knowledge regarding a virtual organization's interdisciplinary collaboration environment may inform practitioners on possible alternative paradigms.

\section{Significance to Theory}

The exploration of interdisciplinary research and development program leadership from the members' perspective effects positive social change by capturing and sharing the voice of the affected participants. Follower viewpoints provide unique insight and perspective to additional targets of social change. Considering the perspective of highly educated participants in complex interdisciplinary teams contributes to current 
organizational and leadership theory. These research results were expected to inform future quantitative studies on leadership strategies and the influences of organizational culture. Research on interdisciplinary research and development programs was in response to a call from past researchers to address the challenges of understanding leadership from a follower's perspective in complex systems. For example, BoschSijtsema et al. (2011) recommended additional areas of research needed to understand the culture, trust-building, and technology use in distributed environments. Garrison, Wakefield, Xu, and Kim (2010) recommended future research on trust, and cohesion leadership processes, against an evaluation of an individual's level of experience. Anand, Hu, Liden, and Vidyarthi (2011) called for additional learning on complex-systems and leader-member exchange theory. Finally, Bligh (2011) called for specific followercentered research. Existing research indicated a need for improved understanding of leader-member relationships, which become more critical in a virtual leadership scenario (Dyer et al., 2013).

\section{Significance to Social Change}

The investigation into the complex virtual leadership of interdisciplinary research and development programs may contribute to a positive social change in management knowledge. Potential modifications in training focused on management for scientific leaders would better prepare them for complex organizational leadership environments. Improved training could lead to a larger pool of leadership resources for interdisciplinary collaborations with an understanding of the current and potential paradigms in scientific collaboration which could have national and global impacts through increased 
innovation. The improved relationships between virtual leaders and their followers participating in multiorganizational scientific collaborations could spur further research into scientific competitiveness and virtual leadership in interdisciplinary teams.

\section{Summary and Transition}

The U.S. Congress has mandated the formation and investment in multiorganizational research and development programs. Significant tax dollars continue to be invested; however, not all of these programs develop the virtual leadership strategies required to encourage innovative solutions to their globally challenging problems. Some virtual teams endured, others did not, and the role of virtual leadership in these organizations was not well known. Leadership and organizational theorists have only begun to consider the constructs of complex virtual team systems. Researchers of scientific collaboration have started to consider interdisciplinary collaborations.

This case study research could enhance understanding of virtual leadership strategies in complex, multiorganizational, research and development programs. Through exploratory interviews, this research provides insights into followers' perspectives on virtual leadership strategies employed in multiple scientific collaboration programs. These programs represented more than 100 research institutions, located across the United States and Europe, working cooperatively to develop solutions for global challenges. Potential participants were drawn from members who participated in the program's research as indicated by their listing as a program member or appearing on program sponsored publications. Followers' perspectives were the basis for exploring virtual leadership approaches. This qualitative study served as a starting point for 
potential future quantitative research. Study results could lead to additional quantitative research on specific variables and their impacts. This study could also improve leaders' and policymakers' understanding of the need for potential scientific collaboration virtual leadership models and metrics.

In this study, I focused on the gap in research on leadership, complex organizations, and scientific collaboration environments. The results can improve the understanding of virtual leadership from the scientific collaboration participant perspective. An investigation into the virtual leadership of these programs provides an improved understanding of these complex systems to inform management and theory. I aimed to provide some insights into the virtual leadership of multiorganizational scientific collaboration programs, but this work could improve the likelihood that future investments in government-funded research and development efforts may result in innovative solutions to challenging problems. The first step in this endeavor was a literature review to understand potential models, instruments, and terminology. Chapter 2 provides a summary of the available literature and highlights the gap in the information about the virtual leadership of multiorganizational scientific collaboration programs. Chapter 3 provides a discussion of the qualitative method and case study design. Chapter 4 includes information on the data collection, analysis, and findings. In Chapter 5, I offer some items for discussion, recommendations, and conclusions drawn from the interviews and exploration of participant input. 
Chapter 2: Literature Review

After an extensive review of available literature on the challenges in leadership preparation for complex multiorganizational research and development programs, I considered three areas of research:

- Research associated with team leadership including leader relationships, virtual leadership, and shared leadership.

- Research regarding complex organizational systems including multiorganizational systems, multiteam systems, and distributed organizations.

- Research focused on the scientific collaboration environment including multidisciplinary teams, collaborative environments, and trust.

In addition to framing the research, I reviewed additional related topics to inform the development of interview questions and coding of interview results. Shared leadership, transformational leadership, and leadership perception were some of the main topics that surfaced associated with team leadership.

To capture the effect of relationships between leaders and their followers within a team, I reviewed leader-member exchange research, a version of social exchange theory (Erdogan \& Bauer, 2014). I chose to investigate this topic because leader-member exchange theory goes beyond the view of the leader to considering the individual relationships between leaders and members. Leader-member exchange theorists have acknowledged that there were multiple relationships between leaders and members (Schermuly et al., 2015). 
I drew on research from the concepts of complex organizational system leadership and the idea of legitimate authority (Jay, 2013). For example, Wageman and Fisher (2014) evaluated four areas in their research on legitimate authority: executing tasks, monitoring, and managing, designing the team, and guiding overall direction. Hazy and Uhl-Bien (2014) described the leadership of these complex systems as a new area of exploration from a leadership perspective. Murase et al. (2014) focused their research on the challenges of multiple team systems, looking at the focus, function, and forms of leadership. They also addressed options, such as centralized leadership versus multilevel vertical leadership, noting the need for new metrics for leadership in these types of multiple team programs. These emerging leadership structures for complex systems contributed to the foundation for the conceptual framework for this study.

This review includes research into scientific collaborations to address the challenges of the interdisciplinary nature of scientific collaborations, often involving highly educated scientists cooperating and functioning as a single program theory (Bozeman et al., 2013; Lariviere et al., 2015). I also considered scientific research behavior in this literature review. Scientific behavior, or culture, included competition for funding and prestige, the scale of collaboration, the organizational structure of collaboration, the technology used, the information interdependencies, and the collaborative relationships (Shrum et al. 2007). This literature review includes the current research in all the areas above. 


\section{Literature Search Strategy}

The conceptual framework for this study arose from emerging research regarding leader-member exchange theory (Schermuly et al., 2015) and complex-systems theory (Cady, 2016; Hazy \& Uhl-Bien, 2015; Long et al., 2013). These theories, along with research on virtual leadership (Hoch \& Kozlowski, 2014) and on multidisciplinary scientific collaboration environments (Bozeman et al., 2013; Lariviere et al., 2015), provided the basis for my literature search strategy.

The approach for this literature search included a variety of research tools. First, I conducted a keyword search on some individual databases through the Walden Library. I used GoogleScholar to search across references, find related articles, and add to the search nomenclature. I also used reference linkages and related journals to extend the search criteria. Once my investigation of the available databases was complete, I engaged Walden Library staff to identify additional databases to investigate. Finally, I explored resources outside of the databases such as government websites, professional organizations, and research groups looking for research on Centers of Excellence programs.

Researching individual databases through the Walden Library yielded peerreviewed articles on organizational management, leadership, complex organizations, virtual leadership, and scientific collaborations. I searched business and management databases such as Business Source Complete, Emerald Management, SAGE Premier, and ScienceDirect, as well as interdisciplinary databases such as ProQuest Central and Academic Search Complete. These interdisciplinary databases contained current research 
on leadership and complex organizations. I searched records, such as Hoover's Company Records, to see if any of the DHS Centers of Excellence programs were under their formal program name. My search included several related subject databases including Political Science Complete. There I found one refereed article on the virtual Centers of Excellence programs (Bohldin, 2013). PsycINFO yielded several articles on leadership. I also found two articles referencing leadership instrumentation in the Health and Psychosocial Instruments database. These instruments were not appropriate for this research, however the terminology found within them contributed to the coding lexicon. I used Google Scholar for general searches, specific author searches, and citation linkages. In addition to peer-reviewed databases, I searched government websites including the DHS (2017), the DOE (2015), and the Environmental Protection Agency sites for interdisciplinary, multiorganizational, and scientific collaboration information. I also gathered information specifically on the DHS. I considered The RAND Corporation's review of DHS testimony submitted to the House Homeland Security Committee, Subcommittee on Oversight and Management Efficiency. Gerstein (2015) provided recommendations for efficiencies in defining the DHS challenges. The RAND Corporation recommended innovative engagement with industry research and development organizations (Gerstein, 2015).

Finally, recommendations from a project briefing with the Director of DHS Science and Technology's Office University Programs yielded additional search terms. The Director suggested additional literature reviews on public choice, incentives and 
culture, and interdisciplinary scientific collaborations. In all, more than 100 articles were collected, reviewed, and synthesized to inform this study.

\section{Literature Review}

In this research, I aimed to learn more about the virtual leadership of interdisciplinary scientific collaborations sponsored through multiorganizational research and development programs. This literature review addresses the gap in current research on leader-member relationships in complex, multiorganizational, scientific collaboration programs. I looked at virtual leadership strategies, the challenge of innovation management across disciplines, and scientific collaboration across organizations using DHS's Centers of Excellence programs as my basis. This research built on the leadermember exchange theory (Schermuly et. al., 2015) as a construct to explore emerging complex system leadership (Day, Griffin \& Louw, 2014; Hazy \& Uhl-Bien, 2014) targeting multiple team systems (Murase et al., 2014; Elfner et al., 2011). I combined insights from existent research on leadership with research on scientific collaborative influences to improve understanding leader-member relationships in complex multiorganization research and development environments (Bozeman et al., 2013). The following literature review covers existent research on team leadership, complex organizations, and scientific collaborations. Figure 1 provides a breakdown of the literature review areas and subareas explored. 


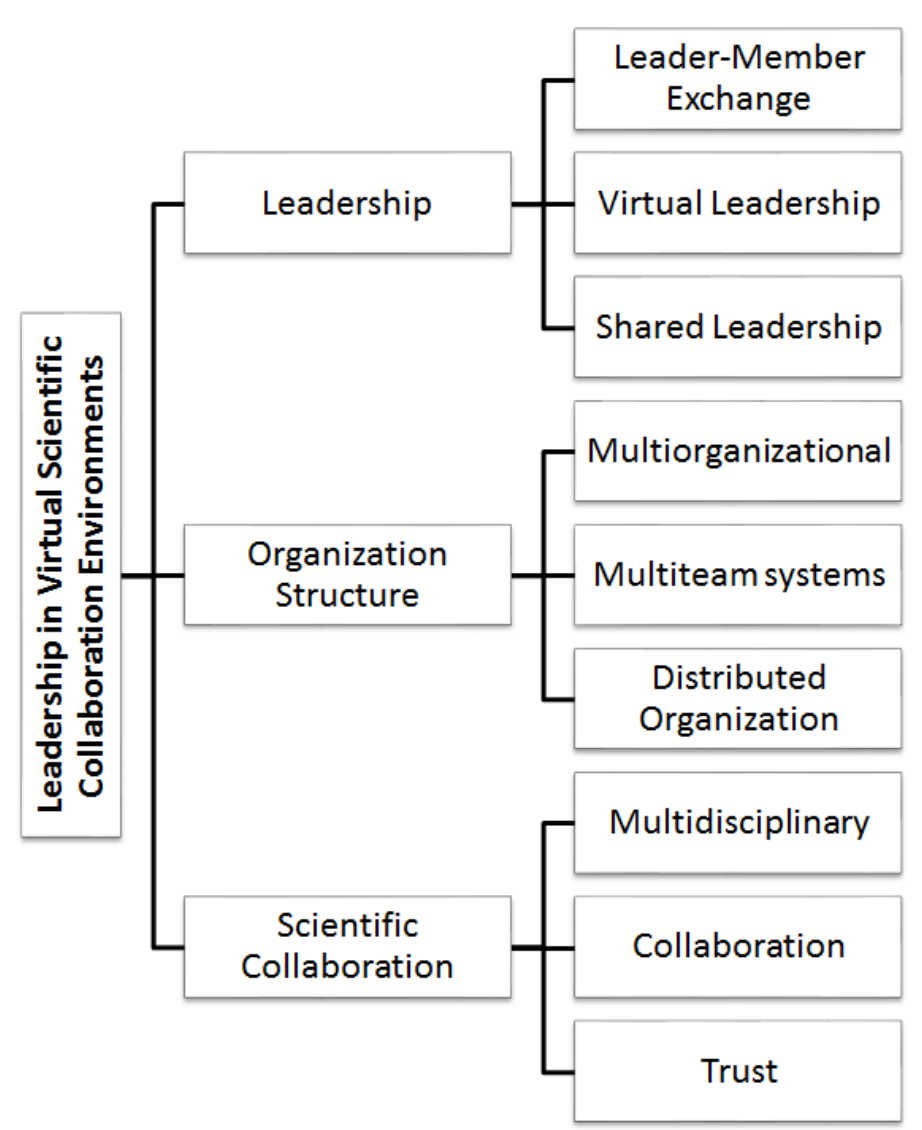

Figure 1. Structure of my literature review.

This literature review was broken down into three main areas including leadership, organizational structure, and scientific collaboration. Each area was then split into subareas of research to extend the depth of the literature review. Figure 1 provides the high level structure subjects for my literature review. I provide a synthesis of material that can inform the exploration of the virtual leadership of interdisciplinary scientific collaborations.

\section{Leadership}

Researchers have called for a more holistic consideration in the leadership of complex multiorganizational systems (Dinh et al., 2014; Henry, 2015). For instance, Dinh 
et al. (2014) studied leadership research, looking for insights into theories and the development of new theories. Leadership research trends in the last decade indicated a growing interest in a need to identifying additional theories to capture leadership observations (Dinh et al., 2014). Most publications identified from top-tier publications such as Leadership Quarterly, Journal of Management, Organizational Science, and others related to leadership have acknowledged the role of leadership in influencing the organization (Dinh et al., 2014). Lee, Martin, Thomas, Guillaume, and Maio (2015) concluded that social identity of the workgroup affected a holistic view of a team, including both leader strategies and follower attitudes. A review of leadership training programs yielded no evidence of leader exposure to these modern concepts.

One way to understand leadership better is through using a common language; Seemiller and Murry (2013) offered a potential coding lexicon to analyze interdisciplinary leadership through their study. Seemiller and Murry studied academic programs in more than 70 academic organizations offering leadership development curriculum to their students. Their goal was to understand leadership competencies across academic disciplines. These researchers also worked to translate their observations into contemporary leadership models, such as relational leadership, social exchange, and emotional intelligence. No one model was sufficient, or consistent, across the variety of academic programs evaluated (Seemiller \& Murry, 2013). Seemiller and Murry’s research primarily gathered and categorized leadership competencies. These researchers focused on defining a common language related to leadership development across 
academic disciplines. They offered a potential coding lexicon to analyze interdisciplinary leadership input from a variety of followers.

Santos, Caetano, and Tavares (2015) extended research into leadership training by going beyond functional behaviors to competencies that included leadership of teams. They compared traditionally trained leaders and their traits to leaders trained in team leadership. Santos et al. found that those trained in team leadership demonstrated more team effectiveness. Situation clarification, strategy clarification, and team coordination were the main three categories of team leadership functions identified as critical to team effectiveness (Santos, Caetano, \& Tavares, 2015).

In this literature review, I first consider the literature on leader-member exchange theory. To capture the impact of the distributed nature of the research and development programs, I provide a review of the research on virtual leadership including possible leadership models. I complete the discussion on leadership by probing deeper into one such virtual leadership model looking at shared leadership.

\section{Leader-member exchange theory.}

Understanding the challenges and many theories associated with leadership will support my decision to use leader-member exchange theory for my research on multiorganizational research and development programs. Northouse (2016) provided a range of leadership theory approaches based on individual leaders' traits, skills, styles, and situational factors. Of these possible leadership models and relationship options, leader-member exchange theory provided a unique perspective that accounts for variability in interaction with followers from the follower's perspective (Schermuly et al., 
2015). The complexity of scientific collaboration programs required a perspective on leadership that includes consideration for the complexity of teams and organizations. Day (2014a) extended leadership processes and patterns to more complex multiorganizational systems. Day (2014b) noted that leader-centered research currently dominates the field and encouraged evaluation of leadership as a process rather than a position. A processcentered view allows extended learning as patterns and methodologies developed. A process-centered view has greater potential to be generalizable as the field of leadership continues to mature (Day, 2014a). Leader-member exchange theory was a viable option to investigate multiorganizational scientific collaboration programs and relationships between collaborators and leadership.

Leader-member exchange theory is a subset of social exchange theory. Leadermember exchange theory goes beyond the individual relationships between any two members of a team and targets the multiple relationships among leaders and followers. Erdogan and Bauer (2014) studied these relationships as a network of relationships and evaluated them for processes and patterns. I viewed leadership as a relationship with multiple individuals rather than the traits of an individual leader. One additional complexity that I considered was the likelihood the relationships change over time (Erdogan \& Bauer 2014). Northouse (2016) summarized leader-member relationship phases using a time continuum. Northouse's continuum had a relationship described as strangers at one end of the spectrum and moved to partnership at the other end of the spectrum. Each phase of Northouse's continuum had a marked change in roles, influences, exchange quality, and interests. Viewing the development of relationships as a 
process would also help anticipate and adjust for changes over time. The added challenge of team relationships existing across organizational boundaries, at different stages, and managed differently for each member, significantly increases the complexity of leadership.

Leader-member exchange theory was designed on the concept that leaders do not have the same relationship with all of their members. Differing levels of trust can affect both the leader-member relationship and the relationships among members (Erdogan and Bauer, 2014). The underlying concept of equality for each follower may only be achievable in theory. A leader may strive to treat all followers equally, but not all followers will be equal in their competence or motivation, nor will they bring the same motivation and personal experiences to the relationship. Erdogan and Bauer (2014) provided a basis for leader-member exchange quality in the relationship, including effect, loyalty, and professional respect. The inclusion of professional respect was of particular interest in the scientific collaboration environment. In summary, leader-member exchange theory helps to acknowledge that individual relationships vary among team members.

I went beyond Graen and Uhl-Bien's (1995) fourth stage of multiple follower relationships with the leader and other followers to include multiple organizational relationships where the leader may not be part of the immediate organization. More current researchers introduced the idea of team networks to address the complex relationships in leader-member exchange scenarios (Long et al., 2013). Long et al. (2013) conducted research using a social networking methodology with graph theory of 
relationships. Fairhurst and Uhl-Bien (2012) proposed the use of organizational discourse analysis to try to capture the leader-member relationship processes. This organizational discourse analysis moved researchers away from linguistic assessment versions of communication analysis to work toward understanding the processes influencing leadership. Collectively, these methods along with those found in scientific collaboration research served as the basis for gathering perspectives from interviews of scientific collaboration program members.

Erdogan and Bauer (2014) noted that the leader-member exchange included leader, follower perspectives, and relationship perspectives. Long et al. (2013) looked at leaders and members, but also the processes and roles in connecting the team members. Long et al. discussed a leader who could be viewed as a manager, or be considered an opinion leader, based on their influence on the overall team. These researchers used semistructured interviews, rather than a defined instrument, to allow more freedom in capturing relationships and influence. Viewing a leader through multiple perspectives has only recently emerged as a possible way to frame the leader-member exchange challenge. Future studies on leader roles would need to continue to flesh out the parameters and methods for understanding this more complex leader-member concept.

Fairhurst and Uhl-Bien (2012) recommend moving away from surveys to encourage interviews as a method for improving understanding regarding the leadermember relationships. The opened-ended nature of interviews offered an opportunity to learn and investigate relationships. Discourse analysis of recorded conversations revealed processes not noted before the investigation. It was often not feasible to conduct 
discourse analysis because of the difficulty in gathering recorded conversations between leaders and their members. Fairhurst and Uhl-Bien noted that extensive coding of indepth interviews could produce enough quality of understanding to warrant discourse analysis. Their recommendations and methods contributed to the interview development, coding, and analysis approach for this study. Fairhurst and Uhl-Bien used three relational analyses, with two leadership concepts for their discourse analysis. Their relational analyses included an interview that indicated interesting interactions in team control, mobilizing to action, and relationship building. In Fairhurst and Uhl-Bien's analysis, relationship building included humor and storytelling. Evaluating leadership engagement can reveal evidence of sensemaking conversations, as well as discussions related to the identity of the individuals (Fairhurst \& Uhl-Bien, 2012). This categorization process provided a framework for coding interview results given my interest in investigating members' perspectives.

Hudson (2013) considered the leader-member relationship as viewed from the perspective of attachment theory. Attachment theory reduced the complexity of teams to the relationships between individuals and how their relationships were cultivated. Hudson found that impacts to the follower relationships with leaders could be rooted in the attachment experiences throughout a follower's lifetime. Hudson's research did not specify characteristics of the follower and leader populations. Hudson suggested education of leaders and followers on attachment theory. He indicated that educational impacts could have a positive effect on the leader effectiveness. 
Hoch and Kozlowski's (2014) included leader-member exchange theory, along with transformational leadership and career mentoring, as forms of hierarchical leadership in virtual teams. Choy, McCormack, and Djurkovic (2016) used leadermember exchange in their analysis of impacts from leader delegation and member participation on job performance. Choy et al. surveyed more than 250 employees within a single organization to understand how a participative approach to the leader-member relationship can affect the overall job performance. A holistic approach to understanding leader-member relationships extends the current understanding by establishing a working relationship with members. Choy et al. went beyond contractual and economic exchanges to include interpersonal.

Uhl-Bien, Riggio, Lowe, and Carsten (2014) focused on the followership of leader-member exchange in their review of the literature. They attempted to draw inferences from the previous research to guide future research and theory development. Current research addressed followership from two angles, a role-based perspective looking from the members' lens, and leadership perspective following a constructionist approach (Uhl-Bien, Riggio, Lowe, \& Carsten, 2014).

Qu, Janssen, and Shi (2015) looked specifically at leadership that inspired creativity in followers. Their research used leader-member exchange methods to evaluate more than 400 leader-follower dyads to understand the impact of transformational leadership. Qu et al. observed that when high creativity expectations were present, transformational leadership was positively related to the creativity of the follower. Qu et al. noted that leaders with a transformational style allowed the follower to exhibit similar 
goals, values, and standards. Qu et al. researched a single organization. Additional research was needed to understand if distributed leader-member combinations would have a similar effect outside of the organization in the research conducted by Qu et al.

A complementary concept of transformational leadership was transactional leadership (Bass 1997). Transactional leadership includes many of the initiating structure aspects identified by Korman (1966). Leadership behaviors such as directing activities through planning, communicating, and scheduling are indicators of initiating structure behaviors (Korman, 1966). Recently, researcher's interested in leadership have drawn on these early concepts to understand the relationship between leaders and their team members. (Gaudet \& Tremblay, 2017).

Huettermann, Doering, and Boerner (2014) took a followers perspective when exploring leadership impacts on team identification. Their qualitative research looked at four aspects of leadership; providing guidance, encouraging involvement, role modeling, and administering teamwork. They encouraged future research for gathering input from the followers' perspective to aid in understanding leadership strategies fostering team identification. Huettermann et al. described guidance as including clarifying goals, defining team boundaries, and directing team members. They distinguished leadership strategies that encouraged involvement through direct interactions with the team. Interactions demonstrated through listening to the team members, addressing the team, and providing motivation surfaced as important. According to Huettermann et al., rolemodeling included leading by example and advocating for team members. Lastly, Huettermann et al. included administrative actions such as organizing meetings and 
facilitating information flow. Huettermann et al. influenced my interview questions and coding methodology for addressing follower-centered data collection and analysis in this study.

Contemporary researchers continue to demonstrate the need to go beyond leadership traits to understand leadership, and opportunities to improve team performance (Lee, Martin, Thomas, Guillaume, \& Maio, 2015; Asrar-ul-Haq \& Kuchinke, 2016). Lee et al. (2015) noted that follower perceptions require a multifaceted investigation of leadership to understand how leadership strategies affect the perceptions of the followers. Asrar-ul-Haq and Kuchinke (2016) took a similar view of the relationship of leadership to member attitudes, but from the leaders' perspective.

Lee et al. (2015) compared leadership research methods, such as leader-member exchange, transformation leadership, and authentic leadership, to methods that included consideration of follower attitudes. Lee et al. found that organizational performance appraisal systems needed to match the expected attitude of the follower to engage the leader as expected. Extant research on leadership mainly addressed how traits related to organizational outcomes, without consideration of the relationships between leaders and their followers (Hazy \& Uhl-Bien, 2015; Henry, 2015). Lee et al. concluded that future research should move away from focusing on developing leaders' skills to also include organizational context, structure, and function as attributes of follower attitudes. Looking at leader-member relationships in a virtual environment was a specific structure considered that might affect the follower attitudes regarding the leadership.

Virtual leadership research. 
Interest has been growing in the use of virtual teams to address the large complex tasks as found in an interdisciplinary environment (Hoegl \& Muethel, 2016). This interest has led to additional research on virtual leadership strategies related to virtual teams (Gilson, Maynard, Yound, Vartianen, \& Hakonen, 2014). In the last 10 years, several factors have emerged as important, such as the type of work conducted virtually, trust, technology implementation, and redefinition of outcomes (Gilson et al., 2014). Schiller, Mennecke, Nah, and Luse (2014) looked at trust and collaboration across institutional boundaries to add to the theoretical knowledge regarding virtual collaboration. Schiller et al.'s research on spanning boundaries was conducted using a virtual world simulation activity. The researchers concluded that trust was strong within organizational boundaries and weaker across organizational boundaries. Similarly, Wadsworth and Blanchard (2015) looked at virtual team leadership as a process very different from face-to-face leadership. They examined influence tactics for both face-to-face and virtual leadership, assessing which was more successful. Wadsworth and Blanchard found that virtual leaders need to spend more time in contact with team members, and highlighted technologies and characteristics of successful virtual leaders. Wadsworth and Blanchard did not address the virtual leadership culture specific to scientific collaborations. It was unclear if scientific collaborators were more comfortable with technology, or if the interdisciplinary nature would influence characteristics, such as empathy.

Hoch and Kozlowski (2014) described the elements of a virtual team as being geographically distributed, dependent on electronic communication, and potentially having varying cultural backgrounds. Serban et al. (2015) conducted quasiexperimental 
research comparing face-to-face leadership to the virtual leadership of teams. These researchers developed a multilevel model that included characteristics of student teams, such as cognitive ability, personality, self-efficacy, and comfort with technology. Serban et al.'s work related the density of network ties among team members to the emergence of leadership. Despite extensive current research on technology impacts in virtual teams, Serban et al. did not find the follower's comfort with technology to be a success indicator for virtual leadership. Serban et al.'s results may not apply to research on virtual interdisciplinary scientific collaboration teams for two reasons. First, their population comprised students, and the inference of student characteristics to those of professional researchers was unclear. Second, this student population was limited to e-mail for communication.

As federally funded consortiums of organizations were established to bring together multiple disciplines into one complex program, the virtual leadership of these complex systems also became important (Sidhu \& Volberda, 2011). One approach to virtual leadership in recent studies was e-leadership (Avolio, Sosik, Kahai, \& Baker, 2014). E-leadership encompassed the challenges of working in a virtual environment and the role of technology in leadership. Sahay and Baul (2015) examined e-leadership more closely by comparing it to concepts of leadership. Sahay and Baul took a behavior look at leadership and noted that leadership and culture were important. Sahay and Baul did not make specific correlations between organizational characteristics and context.Their research considered the impact of e-leadership on organizations in general and concluded that e-leadership was a key element in organizational outcomes. 
Technology integration has been integrated into the workforce. Historically this was referred to as sociotechnical theory (Emery \& Trist, 1965). Emery and Trist's first introduction of sociotechnical theory concerned automation in manufacturing, where technology was rapidly replacing human workers. The remaining human workers transitioned to work that included interaction with the technologies. The sociotechnical theory looks at both the human aspects of a system, as well as the interaction of the human with the technical systems. Technology remains widespread in today's society and has become a critical part of distributed team's coordination (Berry, 2013; Halal, 2013; Wang, Hu, \& Li, 2013). Acknowledging the role of technology in the leadership of multiorganizational scientific collaboration was important to consider when understanding virtual leadership processes.

Current research focused on communication elements related to computer science technology challenges (Denning, 2013; Moe, Aurum, \& Dyba, 2012; Ramos, Vasconcelos, \& Barcelo, 2013). In 2001, the concept of agile software development emerged, formally introducing methods of communication, the frequency of communication, and tools for communication among agile software development teams (Highsmith, 2001). Agile software development processes, and specifically collaboration elements of the original agile approach, were believed to have potential beyond software development (Denning, 2013; Ramos et al., 2013). Manufacturing and sales could benefit from the distributed approach to design and development, and agile's adaptive nature could benefit these industries by providing responsiveness to customers' interests (Denning, 2013). Hilt et al. (2016) conducted a case study on agile development 
methodologies in electrical energy storage systems for automobiles. The shared responsibilities of an agile approach may apply to complex leadership collaborative challenges as well.

\section{Shared leadership research.}

An emerging field related to distributed teams was the concept of shared leadership. Hoch and Kozlowski’s (2014) research noted that shared leadership was related to team performance even in scenarios where the team was collocated. Their research focused on hierarchical leadership, structural supports, shared leadership, and their relationships to team performance. By examining more than 100 teams, Hoch and Kozlowski observed that the more distributed a virtual team was, the more its structural supports affected team performance. This impact extended to reward systems, available communication, and information technology. A holistic research approach was needed to understand leadership processes in virtual teams (Hoch \& Kozlowski, 2014).

According to Hoegl and Muethel (2016), the value of shared leadership can remain unrealized if the leader remains tied to traditional leadership models, demonstrates overconfidence in their leadership role, or fear of becoming unessential. In their research, Hoegl and Muethel focused on the effect of shared leadership when operating in distributed teams, and considered the perceptions of the leader in shared leadership scenarios. The researchers provided strategies for enabling shared leadership that required accepting the new paradigm of shared responsibility, teaming behaviors, respecting member competencies, and encouraging leadership behavior in others. 
Similarly, Hoch and Kozlowski's (2014) research included cognitive team leading, effective team support systems, and behavioral member to member exchange concepts.

In addition to the team and organizational structure, leadership has an impact on the relationship between the leader and the members of a scientific collaboration program. Extant research on leadership addressed leadership traits relate to organizational outcomes (Hazy \& Uhl-Bien, 2015; Henry, 2015). Only a limited number of researchers focused on developing methods for leadership evaluation based on organizational characteristics and context (Vessey et al., 2014). For my study, organizational characteristics and context include virtual organizations, interdisciplinary teams, and multiorganizational collaborations.

\section{Organization Structure}

The organizational structure was one context in which leadership could be investigated further. Historical metrics used for organization impacts were constrained to a single overarching organization (Jay, 2013). Jay (2013) provided a perspective on leadership that included the complexity of a hybrid organization. He described hybrid organizations as a set of entities brought together to spur innovation. Jay noted that these hybrid organizations required new organizational success criteria. Hybrid organizations were a construct considered in exploring the challenges of the interdisciplinary nature of scientific collaborations often involving multiple organizations functioning as a single program. Jay discussed the concept of complex-systems leadership and the idea of legitimate authority, which I evaluated in four areas: executing tasks, monitoring and managing, designing the team, and guiding overall direction. 
More recently, Henry (2015) extended the concept of hybrid organizations to more complex multiorganizational systems. Complex environments can be approached using systems thinking. A systems approach helped focus research questions onto a defined space, allows for flexibility, and guides processes for understanding qualitative data. Henry observed complex organizational environments that needed to be considered a complex system of systems with boundaries identified between systems.

Turkulainen, Ruuska, Brady, and Artto (2015) focused on managing interfaces between projects and organizations. They analyzed program management regarding organizational integration interface options focusing on impersonal, personal, and group interface experiences. Turkulainen et al.'s research was limited to a single organization and not necessarily applicable to multiple organization scenarios.

Edmonstone (2016) provided an examination of Obolensky’s (2014) book on complex adaptive leadership. In his review, Edmonston addressed leadership of complex system emphasizing their changing nature, and their adaptive expectation of leadership. The lines of distinction between leadership and management blurred when chaos and complexity were integral parts of organizational decision-making (Edmonstone, 2016). Hazy and Uhl-Bien (2015) described the leadership of these complex systems as a new area that needs to be explored further from a leadership perspective.

\section{Multiorganizational complexity research.}

Multiorganizational scientific collaboration programs were complex networks of organizations working for a common goal. In my study, the complexity stems from the multiple organizations operating under a single program structure. Added complexity 
surfaced when the product development occurred in a distributed environment. More than 50 years ago, Trist, Higgen, Murry, Pollok (1963) described an organization as a group of 40-50 individuals working together over time who develop social and organizational processes to achieve a common goal and sharing a common mission. In the current research, the importance of the human relationship element of organizational leadership has reemerged as critical to understanding leadership processes in complex systems. More recently, Henry (2015) extended the concept of hybrid organizations to more complex multiorganizational systems. To address the challenges of these multiple organizational constructs, Jay (2013) introduced complex-systems leadership and the challenges of having multiple roles in leadership. Murase et al. (2014) focused their research on processes targeted at the leadership of multiple team systems. These emerging leadership concepts for complex systems supported the framework for this study. Complexity in multiorganizational systems viewed as organizational ecosystems provided a method for relational linkages between organizations. The concept of multiple leadership roles (Jay, 2013) was a method for considering the variety of followers' expectations of leadership.

Marion and Uhl-Bien (2011), suggested that complexity theory may be a method to approach complex adaptive systems, such as a multiorganizational research and development environment. Marion and Uhl-Bien suggested consideration of leadership under such complex adaptive environments needs further research, noting that the context in which the leadership occurred had a significant impact on the way leadership should be studied. A multiorganizational scientific collaboration program, which was my interest, 
provided a very complex and adaptive target for further research. Breaking down complex systems into smaller teams was one way to address this complexity.

\section{Multiple team systems research.}

Organizational theories regarding multiple team systems were an emerging field of study (Chompalov, 2014; Biesenthal \& Wilden, 2014; Turkulainen et al., 2015). Research on multiple team systems could be extended to applications for multiorganizational programs. Murase et al.'s (2014) concept of a multiple team system included the connectivity of the team as a collective organization working toward a common goal. Murase et al.'s noted the goal could not be accomplished independently without the collective cooperation. These researchers noted that each team contributed unique skills. Multiorganizational research and development programs were designed to draw on a diverse set of experts to provide innovative solutions to complex scientific challenges. Murase et al. also confirmed challenges with multiple team system, such as conflict management, cohesion, and communication. In the context of my study, multiple team systems also involved multiple organizations. Murase et al.'s research took a function, form, and focus perspective. There was a need for additional research on multiple team systems and measures of effectiveness (Murase et al., 2014). Another way to view a multiple team system might be to study it as an ecosystem.

Mars, Bronstein, and Lusch (2012) discussed the metaphor of using an ecosystem to understand the elements of complex organizations. Mars et al. described the value of an ecosystem approach and noted that the origin of this concept was not clear. Hanssen (2012) provided a theory of organizational ecosystems related to his concept of a 
software product ecosystem. Mars et al.'s research contained several elements that were similar to interdisciplinary teams, for example, it assumes a variety of roles and expertise to function. Mars et al. included multiorganizational interactions among the elements of the ecosystem. Emery and Trist (1965) noted that organizational environments had experienced a rapid rate of change and increased complexity. These researchers highlighted the need to consider the effects on the organization from the environment outside of the organization. Emery and Trist recommended engaging a systems theory approach to understand organizational behaviors. They noted that organizations experience a transactional interdependency among the organization and the environment around it. More recently, Hazy and Uhl-Bien (2014) and Long et al. (2013) continued to explore the challenges of these complex systems. The perspective of organizational ecosystems brought with it a more complex perspective than a single organization. Although researchers examined trust in distributed teams, they focused on processes of a single organization without taking into account the additional challenges of multiple distributed organizations (Hinds, \& Bailey, 2003; Ocker, \& Hiltz, 2012; Sidhu, \& Volberda, 2011).

\section{Distributed organization research.}

Olson and Olson (2000) discussed challenges faced by distributed teams in their research on collaboration. Their research acknowledged the availability of technology to support distributed teams and highlighted individual connections and communication as a main challenge of teamwork when teams do not collocate. Many of the cautions Olson 
and Olson (2000) put forth more than 15 years ago were still challenges, such as common ground, the coupling of work, collaboration readiness, and collaboration technology.

Geographic separation, differing languages, differing approaches to research, and a competitive culture all worked against a collaborative environment (Daim et al., 2012; Hanssen, 2012). Denning (2010) introduced the concept of extreme management. Extreme management was the term Denning used to encompass the challenges of leading distributed multiorganizational complex teams. He noted that this new complex environment required new methods of leadership. Later, Denning (2012a) proposed a way of thinking about management combined with leadership. He developed an approach to this new management by breaking down leadership into roles, goals, values, coordination, and communication methods. Shao and Muller (2011) put forth a recommendation to understand the success criteria for teams that could be collected together to represent program success. Shrum et al. (2007) focused their research on the challenges of scientific collaboration, and later expanded into types of collaborative structures that might address some of those challenges. In 2011 Sigma Xi leadership decided to evaluate the idea of team science as one of the critical issues in science subjects (Elfner et al., 2011). In their white paper, the Sigma Xi team noted a trend toward interdisciplinary science research that could include teams with individuals from more than one organization. Some research indicated the ability to innovate in distributed multiorganizational teams could lead to scientific breakthroughs. For example, WikiSpeed developed a vehicle that could run 100 miles on a gallon of gas (Denning, 2012b). 


\section{Scientific Collaboration Environments}

Research and development programs can be described as scientific collaborations. These collaborations differ from commercial business environments. For example, in collaborations among universities and national laboratories, most of the participants had advanced degrees. This culture could be analyzed through the lens of collaborative science (Leiserson \& McVinney, 2015). For my study, collaborative science existed within a scientific environment. Funding and prestige were frequent metrics used in these scientific communities. An administrative leader also influenced collaboration through systems and processes. A scientific authority leader directly influenced intellectual collaboration. Maintaining a balance between intellectual leadership and processes was found to be critical to ensure productivity and coordination (Leiserson \& McVinney, 2015).

Mainemelis, Kark, and Epitropaki (2015) shared the perspective that scientific innovation could come from research on leading creative people. Mainemelis et al.'s analysis synthesized prior research on leadership and creativity. These researchers found that a lack of definitions, specific theories, and context-specific observations made it difficult to associate leadership and scientific innovation. It was helpful to look more closely at scientific culture and collaboration (Mainemelis et al., 2015).

Ale Ebrahim (2015) addressed the culture of scientific collaboration and virtual environment through a study of virtual research and development teams, but this was limited to a single organization and organizational culture. It was possible that the simplification of the single organization renders Ale Ebrahim's research insufficient to 
inform leadership strategies for interdisciplinary multiorganizational scientific collaboration programs. The research from the healthcare industry highlighted interdisciplinary technical teams (Freeman, Baurmann, Fisher, Blythe, \& Akhtar-Danesh, 2012; Lalor et al., 2013). The healthcare research did not address research and development team leadership of interdisciplinary collaborations in depth.

\section{Interdisciplinary teams.}

In Kuhn's (2012) discussion of scientific revolutions, he noted that early scientific thinkers were often very close friends with scientists of multiple disciplines. He discussed the separation of disciplines, which occurred when research institutions came into existence, and academic departments became competitors rather than collaborators. Kuhn noted that over time, the ability to communicate between disciplines declined.

Organizational incentives promoted a competitive environment. According to Kuhn, this scientific competition increased when organizations began to compete for research and funding. Years of competing for research dollars had resulted in organizations striving to distinguish themselves from each other (Sanberg et al., 2014; Walsh \& Huang, 2014). These disciplinary silos still exist today, and geographic separation between researchers made communication more difficult. Some industries have begun to look more closely at the challenges of interdisciplinary teaming.

The medical industry was an example of an industry in which researchers had begun to evaluate the benefits of interdisciplinary cooperative environments (Manusov, Ronnau, Vela, Lyndia, \& Galke, 2015; Lalor et al., 2013). Manusov, Ronnau, Vela, Lyndia, and Galke (2015) discussed the silos found in education. They noted the need to 
employ an interdisciplinary approach to improve patient care. Medical industry researchers extended the multiple discipline approach to include multiple organizations. Recent medical industry collaborations include educational institutions, researchers, and general practice physicians (Manusov et al., 2015). Lalor et al. (2013) focused on multiple disciplines in a single organization in his review of different levels of participants in interdisciplinary medical teams.

Bedwell et al. (2012) analyzed the various uses of the concept of collaboration across many disciplines. They determined that collaboration can serve as a higher level construct that houses ideas of cooperation, teamwork, and collaboration. Poirier, Forgues, and Staub-French (2016) began the extension of collaborations and innovation into the architecture, engineering, and construction fields. In their research, Poirier et al. noted that collaboration was not specifically defined and was approached differently by various researchers. For this study, I considered collaboration in the scientific community.

\section{Collaboration.}

Viewing research on multiorganizational research and development program leadership through the lens of scientific collaboration was critical to understanding the scientific collaboration programs. Research and development programs were formed to facilitate the scientific collaboration processes. Innovation in these partnerships often resulted from specialized individuals working collaboratively. In the academic community, these specializations were through advanced education and research experience. This culture could be explored through the study of collaborative science (Leiserson \& McVinney, 2015). The study of collaborative science research 
acknowledged that cultural factors influence successful teaming, and considered the effects of competition for funding. Dailey (1978) highlighted the culture of scientific research and challenges to collaborative problem-solving. Challenges continue to emerge in scientific culture with more teaming environments emerging through open science in competing organizations (Walsh \& Haung, 2014). Some researchers have taken on the scientific culture challenge of collaboration by focusing on investigating the challenges of multiple departments in educational institutions ( $\mathrm{Su}, 2014)$. In this study, I considered the academic culture and highly educated environment of the research and development teams through viewing them as scientific collaboration programs.

Lariviere, Gingras, Sugimoto, and Tsou (2015) took a results view of the value of collaboration by investigating the number of collaborators participating in a publication as an indicator for successful collaboration. The more notable the collaboration among multiple authors, the more there was a perceived impact on consumers of the publication (Lariviere et al., 2015). Lariviere et al. indicated growth in the collaborative scientific environments was complex. Collaborative environments were expected to have an advantage over individual scientists conducting research when measured by their peers in the scientific community. It was important to my study to consider the context of scientific culture when exploring participants' perspectives of their virtual leaders. Extant research on interdisciplinary scientific environments informed the selection of the population sample, coding of interviews, and evaluation of observations. The additional focus on leadership and followership in these collaborative scientific environments was an area not yet explored in multiorganizational case studies. 
Paulsen, Callan, Ayoko, and Saunders (2013) discussed how leaders influenced innovation in scientific collaboration environments. A growing number of multiorganizational research and development programs depended on scientific collaboration. Assembling these collaborations provided an opportunity to encourage novel solutions to challenging problems in energy and water resource management, climate change, healthcare delivery, and national security (Cho, 2013; U.S. DHS, 2017; U.S. DOE, 2014; Moniz, 2012; Su, 2014). Of particular interest to this study was the level of scientific leadership from the perspective of the follower. The followers' perception of the leader's competencies may, in part, consider demonstration of past research in the scientific field. In their case study, Shrum et al. (2007) partitioned the possible roles of the leader into an administration leader and scientific authority. The administrative leader could influence collaboration through systems and processes. The scientific authority leader could directly influence intellectual collaboration. Maintaining a balance between intellectual leadership and process leadership was found to be critical to ensure productivity and coordination (Leiserson \& McVinney, 2015).

According to Shrum et al. (2007), exploring several aspects of scientific partnerships can improve understanding of collaborative environments. The environments included the scale of the collaboration, the organizational structure, the technology, the information interdependencies, and finally the relationships among collaboration members. Scientific collaborative relationships were broken down into trust, conflict, and performance. The scale of a particular research and development program affects the amount of leadership, type of leader, administrative organization, and 
structure that was most effective (Shrum et al., 2007). In their research, Shrum et al. used four metrics for describing the scale of a program; formal contracts, the number of levels of authority, a system of rules, and administrative leaders. Small organizations had fewer layers of authority and fewer systems of rules. The largest organizational programs had more administrative leaders and formal contracting mechanisms to drive the research.

Shrum et al. (2007) looked at the effects of the number of teams and number of organizations participating in the program against the same four scale metrics. These researchers found there was little difference between a large number of organizations and a large number of teams. Likewise, fewer organizations and fewer team assessments were also very similar. Program scale had its pros and cons. Smaller programs allowed better management and more satisfying participants, while larger programs allowed for largescale of research, more participants, and broader organizational participation. The size of the organization was directly related to the organizational structure put in place (Shrum et al., 2007). More recently, Gerstein (2015) of The RAND Corporation discussed the challenges with government organizations engagement in industry research and development partners. Factors such as unclear requirements, collaboration technologies, and contractual challenges were found to inhibit a collaborative culture (Gerstein, 2015). The RAND Corporation's review included an assessment of technology available to DHS to enable working with industry. Gerstein also noted that there were obstacles to using tools effectively to engage industry, even when the tools were available. The RAND Corporation included a discussion on the value of engaging industry and the need to 
articulate requirements clearly (Gerstein, 2015). Actionable requirements were the largest obstacle to DHS and industry engagement (Gerstein, 2015).

Shrum et al. (2007) highlighted the importance of considering organizational structure and metrics when understanding the drivers for and challenges to scientific collaboration. These researchers used the concept of bureaucracy to depict one end of the spectrum where there was a division of labor, hierarchical management, and documented controls. On the other end of the spectrum was the autonomous scientist with free reign to explore and create. Shrum et al. proposed an optimal organizational structure that was flexible, democratic, and allowed for interdependent organizational activity.

Shrum et al.(2007) provided a framework for scientific collaboration that encompassed decision-making hierarchy, the level of formalization, mechanisms for scientific leadership, and division of labor. In the related metrics considered, literature and patents were the most notable types of collaboration products published. Walsh and Huang (2014) researched collaborating scientists from the U.S. and Japan, facing the challenges of collaborating in a competitive scientific environment. Patents and copatents were explored to understand the drivers and barriers to scientific collaboration (Belderbos, Cassiman, Faems, Leten, \& Van Looy, 2014). My study included similar organizational structures and potential metrics for multiorganizational research and development programs. One potential metric for success in the scientific community could be the number of publications. Scientific collaborations of any kind were considered advantageous to researchers when viewed from the publication perspective (Lariviere et al., 2015). Additional measures of performance would need to be part of the 
investigation to understand leadership in multiorganizational research and development programs.

When using multiple elements for participation, a leader's responsibilities could be identified as a performance metric for a scientific collaboration (Shrum et al., 2007). Shrum et al.'s elements included administrative elements and innovative success related factors. Administrative elements were consistent with traditional business administration elements of completion of deliverables on schedule and within budget. The innovative success related element was more challenging because it was dependent on a perception of success, varying definitions of success, and appears to change over time. Shrum et al. took a closer look at the innovative success element of performance and evaluated two areas: resource uncertainty, and data sharing. The results on resource uncertainty were directly related to collaborators perceptions of success. Collaborations initiated with high uncertainty in resource funding were perceived as successful. These less-certain collaborations equated to unencumbered research and resulted in outcomes that were more positive. Shrum et al. noted that the collaborator's expectations might be higher under stable funding conditions. Another reason could have been the continual monitoring of the program by beneficiaries of the products; the public nature of the research over time resulted in more opportunities for scrutiny. In contrast, the research found that less certain collaborations equated to unencumbered research, and resulted in more outcomes that were positive. Shrum et al. also noted the success of collaboration could be perceived even if no noticeable result came from the research. Another observed 
result was that new collaboration programs were viewed as more successful than those well-established programs.

The second aspect of success was the information sharing among collaborators. The ability to measure the success in this area was challenging. To discuss this element Shrum et al. (2007) split the information sharing into multiple parts. First, they considered collaborations that focused on building an instrument with information sharing as the goal. The researchers also considered collaborations where the goal was information synthesis with the intent to prove the existence of a phenomenon. In this research effort, Shrum et al. determined the collaboration's instrument was successful when the results supported the instrument's value itself. In the case where the collaboration information was integrated with the instrument to demonstrate a phenomenon, the research was only viewed as successful if the phenomenon results validated the original hypothesis of the research. In essence, the success of the phenomenon itself indicated the view of the success of the collaboration. The collaboration was not viewed as successful when the collaborative research did not confirm the phenomenon (Shrum et al., 2007). It was important to capture the success factor for collaboration to understand the drivers for leaders' relationship with followers. The variety of ways a collaborative team views success highlighted the need to ask participants for insight into this aspect.

A final perspective that Shrum et al. (2007) shared on success noted the view of those inside the collaboration versus those outside the collaboration itself. Shrum et al. found that it was common for outside perspectives to view larger projects as more 
successful than smaller projects. It was also more likely for international collaboration to be more viewed as more successful than those in a single country. Finally, in contrast to the view of internal participants, Shrum et al. found that those collaborations that engaged a hierarchical structure were significantly more successful than those with consensus-style management. It was clear that success factors differ dramatically depending on the perspective of the assessor.

In addition to collaboration perspectives, Shrum et al. questioned the interdependence of technology sharing and collaboration. They noted technology was often a key driver for scientific collaboration. Costs of scientific computer centers, data centers, and specialized laboratories may have driven a team of scientific researchers towards collaboration for cost savings. Technology could be one of the measurable collaboration points between organizations, which made it a likely target for researchers.

Significant research has focused on communication and collaboration technologies (Berry, 2013; Halal, 2013; Wang et al., 2013). Acknowledging the role of technology in the leadership of multiorganizational scientific collaboration was important to consider when understanding leadership processes. One significant change in the history of scientific collaboration was the development of technology for information exchange and communication. More secure data sharing moved collaboration into a new state where individuals from multiple organizations began to share information and ideas more readily. Previous researchers investigating multiple team collaboration focused heavily on the capability of electronic systems and challenges to distributed network information communication. Their research did not look at multiple team collaboration as 
interpersonal connections between members of a team (Denning, 2013; Moe et al., 2012; Ramos et al., 2013). The human interpersonal communication challenge that went well beyond the software and hardware systems brought with it the need to understand when, why, and how teams interact. Previous research methods needed to be extended to examine the leader-member relationship impacts beyond the communication technology's used to understand the collaborative environment.

Technology and information interdependencies significantly affect scientific collaboration options (Bosch-Sijtsema et al., 2011). Shrum et al. (2007) offer one perspective on technology in a scientific collaboration focused on technologies' role in the collaboration. Their research included technology instruments with applications and models developed by the collaborators for acquisition and manipulation of information. One of the benefits offered for scientific collaboration was to obtain technology that an organization does not already have. For this study, technology included more than the hardware and software associated with data capture and sharing. Technology also included the methods for sharing, capturing, manipulating, and analyzing the information. Shrum et al. broke down scientific collaboration around technology into four types that do not include the social use of technology for communication among collaborators. The most collaborative scenario offered by Shrum et al. was when the technology itself was the focus of the collaborating activity. A copatent collaboration would be an example of a collaboration based on the technology used in the scientific collaboration.

Shrum et al. described another type of scientific collaboration involving sharing the technology for the duration of the collaboration. Organizations agreed to timeshare 
technology to optimize work. In these agreements, the collaborator's information remained independent with technology and security processes being the only collaborative variables. Another technology option in collaboration was subcontracting technology. This level of collaboration only affected communication systems, data stores, or other shared technologies.

The last collaboration provided by Shrum et al. was the case of purchased technology. Purchasing technology was only viable when the technology was not specific to the collaboration and was available commercially. In any scenario, the researchers noted that it was important for scientific collaborators to have a strategy for their information sharing technology use and development. Technology sharing was important to consider in the communication and social connection element of scientific collaboration (Shrum, 2007). The communication and social connection were closely related to trust and trust building behaviors.

\section{Trust.}

Trust was a factor considered in multiple team programs, given the extensive extant research on trust among distributed teams (Bosch-Sijtsema et al., 2011; McNab et al., 2012, Ocker, \& Hiltz, 2012). Schilke and Cook (2013) took a crossorganizational look at trust and provided a model that was potentially extensible to distributed multiorganizational research team leaders. Their model included processes for spanning boundaries and the dynamic nature of trust building. Trust research focused on a humanto-human relationship. My study went beyond human relationships to consider the 
importance of technology to support multiorganizational collaboration. Trust was an indicator of a team's ability to address conflict.

Shrum et al. (2007) directly addressed relationship building among scientific collaborators in three areas: trust, conflict, and performance. The researchers noted that control was maintained over a complex system if significant trust exists. The peer review publication process was a classic example of trust building in the scientific community. Shrum et al. evaluated the concept of trust in complex organizational relationships' citing confidence as a metric used to understand responsiveness. Shrum et al. took a closer look at trust among teams and evaluated trust between scientists and their management. The research results indicated that trust among teams was average, but trust between scientists and management was overwhelmingly low. Trust amongst the scientific community was not shared. In Walsh and Huang's (2014) research on entrepreneurs in scientific research organizations, the behavior of publication secrecy among collaborators affected the willingness to share results. The international nature of Walsh and Huang's research was another factor considered in studying multiorganization scientific collaboration programs. Shrum et al. endorsed the need to evaluate the relationships at a team level when discussing trust and confidence. Leader characteristics investigated by McNab, Basoglu, Sarker, and Yu (2012) included an evaluation of trust and relationship building. The impacts of a leader's time spent and familiarity with members was a common element in teaming literature and could lead to opportunities for improvement (McNab et al., 2012)

Shrum et al. (2007) discussed conflict as an important element for scientific collaboration with several dimensions. The number of organizations involved in the 
collaboration, and the number of projects managed, served as metrics for understanding elements that might relate to conflict. Shrum et al. evaluated the overall conflict between management and scientists. These insights were the basis for the inclusion and use of these data in my study.

The most intriguing phenomenon noted by Shrum et al. (2007) was the relationship of trust, conflict, and success. Shrum et al. noted it was important to realize projects were not equally interdependent, which made it more challenging to draw overarching conclusions. Nonteam members viewed the amount of trust separately from success. In the case of large scientific collaboration case study, collaborators tended to segment off work and funding into subteams. These subteams predominantly did not collaborate with other subteams. Shrum et al. (2007) noted that large-scale projects, such as the DHS's Centers of Excellence programs, were difficult to form and fund. It was time-consuming for the team members to stay connected. If a team was not functioning well, it was also more difficult to terminate once started. Collecting information on the duration of teams was also of interest to my study.

It was important to consider the basis for scientific collaboration, acknowledging research and development programs were complex systems with complex leader-member relationships. A case study approach offered a method to obtain additional information on the role of leadership from the followers' perspective. Additional leadership models in a scientific collaboration were of interest to me for this multiorganizational study. A leadership model put forth by Shrum et al. (2007) was a participatory model that included participatory decision-making with a consensus-based process. It was possible that this 
method was only viable when the scientific group's composition as of single specialty, rather composed of than interdisciplinary research members (Shrum et al., 2007). The most collaborative discipline was found to be particle physics. According to Shrum et al., cultural and ideological disagreements did not derail the collaborative nature of the program with particle physicists. Shrum et al. concluded that wide variability in organizational structure exists. Additional research should be conducted to learn more about the indicators that lead to an optimal structure for a particular collaborative program. One final model reported by Shrum et al. was that of a leaderless collaboration option. In this model, structures, processes, and administrative authorities existed, but no scientific authority existed to make final decisions on technical issues. This model resembled the more modern Agile approach discussed earlier (Ramos et al., 2013). In these cases, the different scientists were called upon for different decisions as needed. The concern highlighted in this leaderless model approach was the potential for lack of intellectual consensus (Shrum et al., 2007).

Scientific collaboration provided a cultural lens through which to consider the leader-member relationships in research and development programs. Contemporary research extended work of Shrum et al. (2007) to understand the leadership and organization constructs in scientific collaboration environments (Chompalov, 2014; Bozeman et al., 2013). Elements of trust, information sharing, metrics, and measures of success all needed consideration when working to understand the relationships between leaders and followers. The complex nature of multiorganizational research and development programs, combined with the challenges of scientific collaboration, offered 
an opportunity for learning more about the leader-member exchange from the perspective of the follower.

No existing studies address the gap in research regarding the virtual leadership of multiorganizational research and development programs, from the participants' perspective. No evidence of research existed targeting virtual leadership strategies and selection criteria for interdisciplinary scientific collaborations sponsored by national research and development programs, such as the DHS's Centers of Excellence programs. With the lack of extant research, I designed a case study approach to learn more about the virtual leadership of interdisciplinary multiorganizational scientific collaboration programs. The following gaps and findings provide evidence of my research.

\section{Gaps in the Literature}

The following gaps have been partially filled by this study:

\section{Gap 1: Holistic Exploration of Leadership}

The first gap identified in my study was a need more holistic exploration of leadership in complex multiorganizational systems. The problem addressed in this qualitative study was the lack of a holistic approach to exploring leadership within complex multiorganizational scientific collaboration programs. Researchers called for a more holistic consideration in the leadership of complex multiorganizational systems (Dinh et al., 2014; Henry, 2015). Extant research on leadership mainly addressed leadership traits (Serban et al., 2015), and how those traits related to organizational outcomes (Hazy \& Uhl-Bien, 2015; Henry, 2015). Even though all elements of my study were not addressed in a single existing research product, I gathered research material 
from a variety of perspectives that collectively make up a complex organizational system concept. The literature regarding overall complex multiorganizational systems fell into the following areas; (a) organizational constructs (Jay, 2013; Schilke \& Cook, 2013), (b) teaming constructs (Ebrahim, 2015; Murase et al., 2014), (c) leadership characteristics (Wadsworth \& Blanchard, 2015), (d) follower perspectives (Uhl-bien et al., 2014; Qu, Janssen, \& Shi, 2015), and (e) scientific collaboration environments (Su, 2014; Walsh \& Huang, 2014).

Leadership research trends in the last decade indicated a growing interest in the need to identify additional theories to capture leadership observations. Day (2014b) encouraged evaluation of leadership. He noted that leader-centered research currently dominates the field and should be considered as a process rather than a position. The current study addresses this gap by listening to the participants' perspective regarding leadership within the programs.

Findings from the current qualitative study extend the literature. My research shows that leadership was viewed predominantly as a shared process including multiple levels of the program organization. Other members beyond the program directors were viewed as leaders by the participants. The functions of leadership were distributed and needed to be viewed holistically to be understood.

The following finding stems from the leadership subtheme. There was a gap in available research applicable to virtual leadership strategies targeted at selection criteria for interdisciplinary scientific collaboration programs like the DHS's Centers of Excellence programs. The available research did not address holistic understanding of 
interdisciplinary multiorganizational scientific collaboration programs. None of the complex multiorganizational research analyzed the members' perspectives. This new research was needed to understand leadership strategies that could guide criteria for future leader selection and training requirements. The present research provided a unique member perspective regarding leadership strategies for fostering creativity, crossing organizational boundaries, and encouraging interdisciplinary collaboration.

\section{Finding 1. Programs implemented a shared leadership model.}

The first finding was that programs implemented a shared leadership model, with transformational and transactional leadership occurring at all levels. Consistent with Qu et al. (2015), participants observed that the director of the program provided the vision. They consistently expressed satisfaction with the top leader's level of charisma, energy, and ability to provide the program vision. Qu et al. noted that leaders with a transformational style allowed the follower to exhibit similar goals, values, and standards.

Similar to the research offered by Wageman and Fisher (2014), I questioned if the leadership within the programs analyzed exhibited centralized leadership or multilevel vertical leadership. This finding addresses the gap in knowledge regarding leadership models and their potential applicability to these complex research and development programs. Suggested research areas identified by previous researchers were consistent with the gap and the findings identified here. Researchers called for a more holistic consideration of leadership of complex multiorganizational systems (Dinh et al., 2014; Henry, 2015) 
My observation of these programs shared leadership model addresses the gap in extant research regarding program leadership from a holistic perspective. My research went beyond the traditional view of management focusing on the identified leaders. Exploring the critical role of graduate and postdoctoral research assistants as early career members' highlights a unique scenario in an academic environment. No extant research was found that discussed the role of such transactional leadership at this early career level within similar programs or complex organizations. The previous literature did not include the observation that graduate and postdoctoral research assistants, while the most early career members of the team, functioned in critical transactional leadership roles. Early career transactional leadership in scientific collaboration poses a new area of investigation.

\section{Gap 2: Multiorganizational Constructs in Scientific Collaborations}

The second gap in extant literature was a lack of research on multiorganizational constructs in scientific collaborations. The problem addressed in this qualitative study was the need for improved understanding of organizational constructs within complex multiorganizational scientific collaboration programs. Literature research areas for this study encompassed organizational constructs as they contributed to the followers' perspectives of leadership in interdisciplinary multiorganizational scientific collaboration programs. The key organizational construct areas of research considered were multiorganizational constructs (Jay, 2013; Schilke \& Cook, 2013), organizational ecosystem (Mars, Bronstein, \& Lusch, 2012), and complex organizations (Hazy \& UhlBien, 2015). Schilke and Cook (2013) took a cross-organizational look at trust and 
provided a model with potential to extend to distributed multiorganizational research team leaders. The model put forth by Schilke and Cook included processes for spanning boundaries and the dynamic nature of trust building. Jay (2013) provided a perspective on leadership that included a consideration of the complexity of hybrid organizations. Hybrid organizations were those groups of diverse organizations who were brought together to spur innovation. Jay also noted that these hybrid organizations require new organizational success criteria because historical metrics were focused on a single organization. As multiple organizations came together to make a complex system, research on these complex organizational ecosystems emerged but did not address collaborative scientific environments (Mars et al., 2012).

Hanssen (2012) provided a theory of organizational ecosystems modeled after the early development of a software product ecosystem. Emery and Trist's (1965) explored organizational environments highlighting organizational change many years ago. The challenges related to these complex changing systems still exist today (Hazy \& Uhl-Bien, 2014; Long et al., 2013). Organizational ecosystems brought in a more complex viewpoint than a single organization by including technology (Bosch-Sijtsema et al., 2011), processes, and politics (Ellen, 2014). There was substantial research focused on communication among participants in collaborative settings. These collaborative communication systems also had computer technology challenges (Denning, 2013; Moe et al., 2012; Ramos et al., 2013).

Edmonstone (2016) addressed leadership of complex systems emphasizing the changing nature of these systems. Edmonstone was interested in the adaptive expectation 
of leadership of complex organizations. Recent studies have addressed the challenges of program management across multiple organizations focusing on program success (Henry, 2015; Manning \& Roessler, 2014; Turkulainen et al., 2015). Organizational construct research alone was not sufficient for understanding the challenges of leaders in interdisciplinary multiorganizational scientific collaboration programs.

Team constructs was also a common focus of literature. Luciano et al. (2015) noted the need for new metrics for leaders in multiple team programs. Hazy and Uhl-Bien (2015) described the leadership of these complex systems as a new area needing further exploration from a leadership perspective. In addition to organizational constructs, team and team leader research was also a target for potential evidence of previous research applicable to my study. Team constructs were an element of characteristic assessments of the leadership of interdisciplinary multiorganizational scientific collaboration programs. This literature review found evidence of extant research in virtual teams (Daim et al., 2012; Ebrahim, 2015; Pinar, Zehir, Kitapci, \& Tanriverdi, 2014), distributed teams (McNab et al., 2012; Ocker \& Hiltz, 2012), and multiple team systems (Murase et al., 2014). Theories regarding multiple team systems were an emerging field of study (Chompalov, 2014; Biesenthal \& Wilden, 2014; Turkulainen et al., 2015). Researchers focused on the evaluation of boundaries between teams, suborganizations, and organizations (Henry, 2015; Turkulainen et al., 2015). The virtual nature of these teams came from a distributed construct that implies additional leadership process, communications, and team building challenges. Existent research provided a variety of choices for constructs of these programs. 
Findings from my study extend the literature showing that scientific collaboration constructs may not be as cross-disciplinary as presented in the online program material and as requested in the U.S. Congressional mandate. The cross-disciplinary nature could be misleading during formation and investment in these research and development programs. Understanding the norms and constraints of these programs can improve the opportunity to implement more virtual and interdisciplinary methods.

\section{Finding 2. Programs focused on applied research.}

The second finding was that programs focused on applied research resulting in organizational structures segmented by discipline. My findings highlight the need for additional research on characteristics of organizational structures within scientific collaborations. Funding and discipline surfaced as important to understand impacts on the virtual nature and interdisciplinary nature of the collaborations. The scientific culture explored in my literature review noted impacts from competition for funding and researcher prestige. According to Kuhn (2012), this scientific competition increased when organizations began to compete for research and funding. The study of collaborative science research acknowledged cultural factors influence on successful teaming, and considered the effects of competition for funding (Leiserson \& McVinney, 2015). In my study of large scientific collaborations, programs tended to segment off work and funding according to the technical area. These subteams infrequently collaborated with other subteams. Shrum et al. (2007) noted that large-scale projects, such as the DHS's Centers of Excellence programs, were difficult to form and fund. 
The finding that programs appeared to organize around technical areas and therefore around specific disciplines addressed the question of how current collaborative programs in this study were arranged. The dependency on graduate and postdoctoral research assistants to conduct the bulk of the work appeared to necessitate a more singlediscipline partitioning of work. Dailey (1978) noted that the culture of scientific research contributes to its challenges to collaborative problem-solving. Competitive challenges continued to emerge in scientific culture with more teaming environments emerging through open science (Walsh \& Haung, 2014). With no current research on program structures, this finding indicates that scientific collaboration programs continue to be organized by discipline. A related topic in this gap analysis was the virtuality of those organizations participating in the programs.

\section{Gap 3: Virtual Leadership Across Organizations}

A third gap partially addressed in my qualitative study was the need for insights into the virtual leadership of scientific collaboration programs. Researchers noted that challenging collaboration elements important to overcome were geographic separation, differing languages, and differing approaches to research (McNab et al., 2012; Ocker \& Hiltz, 2012). These elements, along with a competitive culture among locations, were traditionally studied independently (Daim et al., 2012; Hanssen, 2012). Bosch-Sijtsema et al. (2011) recommended future research on areas affected by a leader's interaction with members to understand the culture, trust building, and technology use in distributed environments. The challenges faced by distributed teams working towards a unified product were common ground, the coupling of work, collaboration readiness, and 
collaboration technology (Jirotka, Lee, \& Olson, 2013). Extant research on distributed teams provided some insights into virtual teams. An improved understanding of leadermember relationships was needed as it became more critical in a virtual leadership scenario.

Research on virtual leadership strategies related to virtual teams has emerged to be important over the last 10 years. Researchers found that trust within the team, the type of work conducted virtually, technology implementation, and redefinition of outcomes were all factors in virtual leadership (Gilson et al., 2014). Schiller et al. (2014) looked at trust and collaboration across institutional boundaries, adding to the theoretical knowledge regarding virtual collaboration. Although Schiller et al.'s research on boundary spanning was conducted using a virtual world simulation approach; they found that trust was stronger within organizational boundaries more than across organizational boundaries. Similarly, Wadsworth and Blanchard (2015) looked at virtual team leadership as a process very different from face-to-face leadership by examining influence tactics for both face-to-face and virtual leadership. Wadsworth and Blanchard highlighted technologies and characteristics of successful virtual leaders. they found the need for virtual leaders to spend more time, but did not address the virtual leadership culture specific to scientific collaborations. It was unclear if scientific collaborators were more comfortable with technologies, and if interdisciplinary nature influenced the characteristics studied by Wadsworth and Blanchard. Ale Ebrahim (2015) addressed the culture of scientific collaboration and virtual environment through a study of virtual research and development teams. In this research, the population consisted of a single 
organization with common organizational culture. It was possible the simplification of the single organization renders Ale Ebrahim's research insufficient to inform leadership strategies for interdisciplinary multiorganizational scientific collaboration programs.

Serban et al. (2015) conducted quasiexperimental research comparing face-to-face and virtual leadership of teams. These researchers developed a multilevel model related to the density of network ties between team members and the emergence of leadership. The characteristics of student teams studied were cognitive ability, personality, selfefficacy, and comfort with technology. Serban et al. found that follower comfort with technology was not an indicator of successful virtual leadership.

Recent studies described virtual leadership as e-leadership (Avolio et al., 2014). The subject of e-leadership was an acknowledgment of the intricate dependency that virtual leadership has on technology. Technology continues to be more widespread in today's society and has become a critical part of distributed team's interaction and coordination (Berry, 2013; Halal, 2013; Wang et al., 2013). Acknowledging the role of technology in the leadership of multiorganizational scientific collaboration was important to consider when understanding leadership processes, but it did not address all of the elements necessary to understand virtual leadership of my study. Sahay and Baul (2015) recently looked more closely at e-leadership by comparing it to concepts of leadership. Sahay and Baul's research focused on the leader's behavior in e-leadership environments. Noting that leadership and culture were important, Sahay and Baul did not make specific correlations between organizational characteristics and context. These researchers considered the impact of e-leadership on organizations in general, with organizational 
outcomes as an important metric. In an electronic environment, trust building was important to consider. Additional investigation into trust building was part of the literature reviewed.

Research investigated related to trust and relationships building in four areas of research: teaming literature (McNab et al., 2012), leadership preparedness (Santos et al., 2015; Seemiller \& Murray, 2013), shared leadership (Hoch \& Kozlowski, 2014; Hoegl \& Muethel, 2016; Muethel \& Hoegl, 2013), and characteristics necessary for complex adaptive leadership (Edmonstone, 2016). Collinson (2014) suggested considering new angles from which to conduct leadership research. Collinson recommended seeking to understand the leadership constructs by reconsidering the dichotomies that often surface in leadership theory. Cady (2016) used a system of systems lens to aid in understanding the challenges of complex leadership. Regardless of the view of leadership theory, the extant research did not collectively address the subject of leadership, complex-systems, and collaborative scientific environments.

Limiting the number of virtual interactions themselves added to the literature an example of the challenges faced by virtual teams. Limiting the virtual nature of teams may reduce the dependency on novel communication and relationship building, but also limits the opportunities for collaboration. Several researchers suggested a need for new ways to look at the challenges of a complex system and considered the added challenge of virtual leadership (Avolio et al., 2014; Serban et al., 2015). Team constructs were one potential new way to consider virtual leadership research. My study highlighted 
additional insights on scientific collaboration programs and current structures may deter virtual collaborations.

\section{Finding 3. Collaboration versus coordination.}

The third finding was that collaboration occurred within collocated teams and coordination occurred between virtual partners. My findings indicated that cross-team interaction occurs primarily face-to-face in the initial and final phases of research. The virtual collaboration was limited. This finding contributes to the extant research by noting the avoidance of virtual collaboration. My research may serve as a starting point to explore the potential factors for the lack of virtual collaboration behavior. Wadsworth and Blanchard (2015) concluded that virtual leaders needed to spend more time in contact with team members, and highlighted technologies and characteristics of successful virtual leaders. Serban et al. (2015) conducted quasiexperimental research comparing face-toface and virtual leadership of teams. These researchers developed a multilevel model related to the density of network ties between team members and the emergence of leadership. The solution found in the DHS Centers of Excellence programs appears to be a separation of funding and technical work into collocated subteams. The initial proposal writing and planning occurred across organizations, but the bulk of the work was conducted in smaller collocated teams.

Consistent with Shrum et al.'s (2007) collaborators who also tended to segment off work and funding into subteams. These subteams predominantly did not collaborate with other subteams. Shrum et al. noted that large-scale projects, such as the DHS's Centers of Excellence programs, were difficult to form and fund. It was time-consuming 
for the team members to stay connected. If a team was not functioning well, it was also more difficult to terminate the team once started (Shrum et al., 2007). This finding indicates that the DHS Centers of Excellence programs may have achieved the scale and complexity discussed by Shrum et al. (2007). Shrum et al. did not indicate a particular size or complexity criteria so this finding contributes to the literature regarding a set of programs, their size, and observations in organizational structure.

\section{Gap 4: Scientific Collaboration Environments}

The forth gap addressed in my qualitative study was the lack of current research regarding complex multiorganizational scientific collaboration programs. Critical to this gap was the lack of research from the program participants' perspective. It was important to consider the followers' choice to engage leaders to go beyond leadership characteristic studies (Northouse, 2016). My study considered followers' expectations (Uhl-bien et al., 2014; Qu et al., 2015) and the relationship between leaders and followers (Schermuly et al., 2015). Hudson (2013) used a lens of attachment theory to understand the leadermember relationships. Hudson found that attachment experiences throughout a follower's lifetime appear to affect follower relationships with leaders. It was not clear if the scientific collaboration environment had a different impact on leader-follower relationships. Unfortunately, the research did not specify characteristics of the follower populations. Insights drawn from extant research, combined with research on scientific collaboration influences, could improve understanding of the leader-member relationship in complex multiorganization scientific collaborations (Bozeman et al., 2013) from a follower perspective (Huettermann, Doering, \& Boerner, 2014). 
To address the challenges of the interdisciplinary nature of scientific collaborations involving highly educated scientists from multiple disciplines functioning as a single team, this review included research into scientific collaboration theory (Bozeman et al., 2013; Lariviere et al., 2015) and scientific culture (Bozeman et al., 2013; Jirotka et al., 2013). The scientific culture was used to describe scientific research environment. The scientific culture explored in my literature review noted impacts from competition for funding and researcher prestige. Paulsen et al. (2013) discussed how leaders affect innovation in scientific collaboration environments specifically in the role of transformational leadership. Research regarding scientific collaborations did not consider the perspectives of the members, nor their relationship to the leaders. Theorists addressed the nuances associated with highly educated participants in research communities, but not the complex multiorganizational challenges of scientific collaboration programs (Vessey et al., 2014; Walsh \& Huang, 2014). Vessey et al. described the leadership of scientists as a challenge of leading creative individuals. Mainemelis et al. (2015) shared the perspective that scientific innovation could come from research on leading creative people. Each study on scientific collaboration considered a different angle of the relationship among researchers, although none specified a leader-member relationship extending to interdisciplinary scientific collaboration teams.

Findings from my qualitative study extend the literature showing academic environments have changed little over the last 10 years of research on scientific 
collaboration environments. These findings provide a more current case study specific to the collection of DHS Centers of Excellence programs.

\section{Finding 4. Program members were primarily self-motivated.}

The fourth finding was that program members were primarily self-motivated with publishing serving as evidence of respected behavior. This finding supports the observations of past researchers, noting historical competition for research dollars has contributed to organizations striving to distinguish themselves from each other (Sanberg et al., 2014; Walsh \& Huang, 2014). The study of collaborative science research acknowledged cultural factors' influence on successful teaming, and considered the effects of competition for funding. Dailey (1978) highlighted the culture of scientific research and challenges to collaborative problem-solving. Some researchers took on the scientific culture challenge of collaboration by focusing on investigating the challenges of multiple departments within educational institutions ( $\mathrm{Su}, 2014)$. In my study, this finding contributes to the body of research on highly educated team environments by providing examples of current scientific collaboration program participants' motivations.

This finding contributes to the gap in research suggesting an approach considering leadership as a process rather than a role. Key to the leadership process was relationship building. In Fairhurst and Uhl-Bien's (2012) analysis, relationship building included humor and storytelling. Their relational analyses included an interview indicating interests in interactions of team control, mobilizing to action, and relationship building. Shrum et al. (2007) directly addressed relationship building among scientific collaborators in three areas: trust, conflict, and performance. Additional 
recommendations for research on leadership and complex systems existed in areas such as scientific prestige and culture (Walsh \& Huang, 2014).

My finding contributes to the gap identified on motivational factors for highly educated participants within multiorganizational scientific collaborations. Paulsen et al. (2013) noted that researchers interested in scientific collaboration tend to take the easy road of simply assessing the number of publications and contributors, as a measure of success. Paulsen et al. cautioned that a qualitative approach was necessary. He wanted to improve the understanding of the constructs of scientific collaboration. Including the concept of impact in my finding addresses, Paulsen et al. recommended a qualitative research method be implemented to understand the appropriate measure of success under interdisciplinary collaborative environments. This finding was complementary to researchers addressing the behaviors of academic scientists' and their choice of affiliation on research projects (Su, 2014).

\section{Gap 5: Lack of Leadership Training}

The gap in research and key management issue was a lack of leadership training for scientists taking on the leadership of complex collaborations (Leisrson \& McVinney, 2015). These leaders may not have the necessary experience to lead distributed interdisciplinary multiorganizational scientific collaborations. Seemiller and Murry (2013) reviewed 475 academic programs targeted at leadership and available leadership education for students in general. Jirotka et al. (2013) suggested investment in educational organizations provided a newer element of scientific collaboration for technology design specifically targeted at the challenges of social science in collaborative 
environments. Seemiller and Murry's research did not address which students may take leadership training and why they do. Their research did not provide sufficient information on student choice to understand the decision factors. The student population participating in the program studied was another factor limiting the applicability of Seemiller and Murry’s research. Chompalov's (2014) discussed scientific collaboration training targeted at university leadership, rather than interdisciplinary collaborative team leadership. Scheffer et al. (2015) noted that functioning as a collaborative leader was a recent phenomenon requiring more than one approach to thinking and strategy. Scheffer et al. suggested an additional investigation into collaborative leadership phenomenon.

Santos et al. (2015) addressed the effects of leadership training on team effectiveness. Researching the effectiveness of the overall team with trained and untrained leaders, Santos et al. showed that training has a significant impact on successful leadership. Santos et al. did not address the complexities of virtual leadership, nor the added context of interdisciplinary scientific collaborations.

Collectively the above four findings contribute to the research body of knowledge and may guide training to improve virtual leadership opportunities. It is important to take my findings collectively when considering training and future scientific collaboration programs. Transactional leadership may provide learning-by-doing opportunities, however, it may also limit the leadership knowledge gained through the experience to single organization teaming structures. My research acknowledged the importance of shared leadership, addressing program structure limitations, and potentially missed opportunities for virtual collaboration. These findings together begin to address the gap in 
training needs, and expectations for virtual leadership, within complex interdisciplinary multiorganizational research and development programs.

\section{Summary and Conclusions}

This literature review covered three dimensions relevant to the leadership of multiorganizational research and development scientific collaboration. I discussed research into multiple organizations operating as a single program making up a complex organizational system. I highlighted several constructs for addressing multiple teams, virtual teams, distributed team leadership, and organizations. Possible leadership approaches and constructs, such as leader-member relationship theory, were potential applications for these complex systems. No previous research covered all three dimensions in a single study, yet many studies covered more than one aspect. Responding to congressional mandate, the DHS's Centers of Excellence programs were responsible for encouraging cross-organizational research and development for more than 10 years (U. S. DHS, 2017). The DOE followed suit 5 years ago by introducing the concept of Innovation Hubs (Cho, 2013; DOE, 2014; Moniz, 2012). Each of these programs aimed at encouraging multiorganizational teams to be brought together for collaboration and innovation.

The complexity of these multiorganizational program environments stemmed from both interdisciplinary and crossorganization boundaries. In Kuhn's (2012) discussion on scientific revolutions, he noted that early scientific thinkers were often good friends with other scientists from multiple disciplines. He discussed the separation, or silos, introduced as educational institutions conducting scientific research emerged, 
and departments became competitors rather than collaborators. Kuhn noted that over time, the scientists lost the ability to communicate freely among disciplines. Kuhn also noted that incentives promoted a competitive environment. This scientific competition was more pronounced among research and development organizations over time. Years of competing for research dollars has resulted in organizations striving to distinguish themselves from each other (Sanberg et al., 2014; Walsh \& Haung, 2014). These disciplinary silos still exist today. Geographic separation of team members made communication a challenge.

As federally funded coalitions, research and development organizations were established to bring together multiple disciplines into a single complex scientific collaboration, the resulting leadership of these complex systems was important (Sidhu \& Volberda, 2011). Not all of these collaborations were successful. Researchers do not agree on a measure of success in these scientific collaborations. Paulsen et al. (2013) noted that researchers interested in scientific collaboration tend to take the easy road of simply assessing the number of publications and contributors, as a measure of success. Paulsen et al. cautioned that a qualitative approach was necessary to understand the constructs of scientific collaboration and qualitative research methods as a way to understanding the appropriate measure of success under interdisciplinary collaborative environments. Shrum et al. (2007) found that two driving success factors were the stability of funding combined with duration of the collaborative relationship. Shrum et al. also found that collaborations produced a more successful product than collaborations 
when gathering knowledge or produced results. Lariviere et al. (2015) found that the size of the collaboration affected success.

Leadership theories, such as transformational leadership and leader-member exchange, were also in the literature related to collaboration and complex organizational systems. However, the leadership constructs with their impacts on the success of these multiorganizational research and development programs were not understood. The followers' perspectives were not part of the existing research. Recently, studies addressed the challenges of program management across multiple organizations included looking at program success and different management structures (Chompalov, 2014; Turkulainen et al., 2015).

Organizational theories regarding multiple team systems were an emerging field of study (Chompalov, 2014; Biesenthal \& Wilden, 2014; Turkulainen et al., 2015). New research to understand these organizational constructs strived to consider elements of these complex systems. Some constructs considered in this literature review were virtual teams (Daim et al., 2012), autonomous work teams (Oh, 2012), knowledge-based work teams (Bosch-Sijtsema et al., 2011), temporary organizations (Garrison, Wakefield, Xu, \& Kim, 2010), and distributed teams (Ocker \& Hiltz, 2012).

Challenges faced by distributed teams working towards a common product where discovering common ground, the coupling of work, collaboration readiness, and collaboration technology (Jirotka et al., 2013). Geographic separation, different languages, different approaches to research, and competitive culture all worked against a collaborative environment (Daim et al., 2012; Hanssen, 2012). Shao and Muller (2011) 
put forth a recommendation for understanding the success criteria for interdisciplinary members. These criteria could be collected together to represent a program. The leadership of Sigma Xi evaluated the idea of team science as one of their critical issues in science subjects (Elfner et al., 2011).

Current researchers were heavily focused on communication elements related to computer technology challenges (Denning, 2013; Moe et al., 2012; Ramos et al., 2013). Fewer researchers focused on the management challenges and team communication (Murase et al., 2014; Hazy \& Uhl-Bien, 2015). Agile software development brought a set of processes to collaboration enabling distributed research teams to engage in collaboration (Ramos et al., 2013). These processes were for small working groups, however, many of the principles behind them were extensible to other fields of collaboration (Denning, 2013). Existing research from other industries, such as the interdisciplinary collaboration in health care, provide some insights into the leadership of teams. The views into collaboration insight shared were most often a single organization, or an organization relationship to suborganizations (Freeman et al., 2012; Lalor et al., 2013). Research that took into account the leadership on distributed teams focused on single organizations without taking into account the additional challenges of multiple organization programs (Ocker, \& Hiltz, 2012; Sidhu, \& Volberda, 2011). Hanssen's (2012) research on software ecosystems offered a methodology for conducting the research and communicating the research results. The structure and order of Hanssen's research were a good example of clearly communicating research approach and results. 
It was important to understand leadership from the follower perspective to guide targeted change in the scientific collaboration environments. Understanding leadermember relationships in these multiorganizational programs provided a starting point for focusing opportunities for change. Bosch-Sijtsema et al. (2011) recommend additional areas of research in distributed environments to understand the culture, trust building, and technologies used. Garrison et al. (2010) recommend future research on trust and cohesion leadership processes with an evaluation of an individual's level of experience. The leadership challenges in designing processes for building trust was a common theme in current literature regarding distributed teams consistent with leader-member exchange theory (Bosch-Sijtsema et al., 2011; McNab et al., 2012; Ocker, \& Hiltz, 2012). The impact of the amount of time team members spent together was another common element discussed in the research leading to process opportunities for improvement (McNab et al., 2012). Sidhu and Volberda, (2011) noted that future research needs to go beyond a single organization, and should include seeking an understanding of the role of the leadership in distributed environments. They also suggested future research include the impacts of politics and its effect on the dynamics of leader-member relationships.

The collective literature review helped to inform the methodology applied to my study. I identified four specific gaps in the literature when asking, what virtual leadership strategies do leaders of interdisciplinary collaboration programs use to motivate their highly educated scientists across organizations? For each of these gaps, a finding was provided to add to the body of knowledge regarding the virtual leadership of multiorganizational research and development programs. Finding 1 showed that DHS 
Centers of Excellence programs explored implemented a shared leadership model with transformational and transactional leadership as important facets. Finding 2 showed these programs focused on applied research resulting in organizational structures segmented by discipline. Finding 3 indicated collaboration within collocated teams and coordination occurred between virtual partners. Finding 4 highlights the belief that program members were primarily self-motivated, with publishing serving as evidence of respected behavior. Chapter 3 provides a detailed description of the methodology, rationale for the study design, and planned analysis. 
Chapter 3: Research Method

This qualitative case study, representing the collection of U.S. DHS's Centers of Excellence programs, was comprised of interviews with program members with experience in these scientific collaboration programs. The sample was drawn from individuals from a variety of DHS Centers of Excellence programs to obtain maximum heterogeneity in the sample. Through exploratory semistructured interviews, I drew insights into leadership constructs. Each scientific collaboration program was made up of a consortium of university and research organizations. The DHS Centers of Excellence programs collectively included more than 100 U.S. and foreign organizations, with more than 1,000 individual participants between them. These programs were targeted at specific and applied research areas such as terrorism risks, visualizing data, transmittable diseases, and coastal security.

The research methods I considered stemmed from the following research question: What virtual leadership strategies do leaders of multiorganizational scientific collaborations use to engage the interdisciplinary and highly educated scientists members? The followers' perspectives in this scenario offered a lens through which I explored the strategies of program leaders. To explore complex interdisciplinary scientific collaboration in the multiorganizational context, I considered several qualitative research designs, including case study, phenomenology, grounded theory, and ethnography.

A case study is an option for complex open learning (Yin, 2014). Significant research in virtual leadership in multiorganizational research and development programs 
did not exist to guide the development of a survey instrument. A case study approach allowed for open-ended interviews as a method of gathering information about followers' perspectives on leadership. I chose to use a case study design to provide an opportunity for an open exploration of the multiorganizational program leadership in a complex interdisciplinary organization. In the following sections, I provide details on the research designs considered, the rationale for selecting a case study design, a discussion of the methods planned, issues related to participant selection, and evidence of analysis trustworthiness.

\section{Research Design and Rationale}

A case study research design for my study facilitated exploration of a collection of complex multiorganizational research and development programs focused on solutions for significant global challenges. In the following discussion, I highlight the methods considered and the rationale for the selection of a qualitative method. Included in this section is the basis for the use of a case study design with interviews as a primary process for conducting the research.

Both quantitative and qualitative methods were potentially appropriate for distributed multiorganizational programs conducting collaborative interdisciplinary research. The open-ended nature of the research question fit a qualitative versus quantitative methodology. A potential future application of exploration findings on this topic could result in the development of a quantitative assessment instrument. A qualitative method allowed for open-ended exploration of followers' perspectives on leadership within the context of the phenomenon. 
There were several types of qualitative research methods appropriate for this study: phenomenology, ethnography, grounded theory, systems theory, complexity theory, and case study. I considered a phenomenological design. A phenomenological design could have allowed me to focus on the need to capture a variety of perspectives from multiple members of the same distributed multiorganizational program to balance the perspectives of the interviewees. For my research, some understanding of the experiences of individuals from more than one program was valuable in helping to understand the phenomenon. The organizational context could have been combined with the nature of the collaborative interdisciplinary environment to understand if a phenomenon was program-specific or systemic. Because the phenomenon of interest was leadership, a variety of perspectives from followers across a set of programs was preferred. With the preference of multiple programs, I did not select the phenomenological approach.

Ethnography was a potentially appropriate design for my research on multiorganizational research and development programs. The ethnography processes typically involve observation of the group over time. The program members of interest were not collocated as a single entity, making observation a challenge in my study. The distributed nature of the program members as well as the asymmetric nature of the teaming made observation difficult, if not impossible. I did not select ethnography as a feasible approach for this qualitative design research.

A grounded theory approach would have allowed for an open-ended investigation of programs and permit an analysis of the team environment without requiring an entire 
team to be present for the research data collection. The grounded theory approach offered the ability to build basic theory when extant research or theories did not yet exist. For my study, previous research existed on distributed team environments and multiple organization leadership. This research was sufficient for the development of a conceptual framework for virtual leadership in multiorganizational programs and did not warrant extensive additional theoretical development. Without the need for additional theoretical development, I did not select grounded theory approach for this research.

Complex environments can be approached using systems thinking. The use of systems thinking helps focus research questions into a defined space allowing for flexibility and guiding processes for understanding qualitative data. Henry (2015) observed that complex organizational environments needed to be considered a complex system of systems with boundaries between systems outside of the organization. Emery and Trist (1965) recommended engaging a systems theory approach to improving the understanding of the organizational behaviors. Emery and Trist noted that organizations experience a transactional interdependency between the organization and the environment around it. This environmental interdependency came from the organization's ability to control its processes. There was also an acknowledgment of uncontrolled environments outside of the team that affected the team. The multiorganizational nature of the research and development programs studied here, included an interdisciplinary scientific community that resulted in a complex virtual organization. In my research design, the overlap in complexity and systems theories enabled me to integrate them into my case study approach. 
A case study design encompassed much of the systems theory expectations. Case studies meet the open-ended criteria for an exploratory research design. A case study is a research guided by prior theoretical foundations (Yin, 2014). In this way, it was distinctive from grounded theory. A qualitative case study design appeared to be appropriate for learning more about followers' perspectives of virtual leadership strategies applied in multiorganizational scientific collaborations. A qualitative case study design was chosen to engage the interdisciplinary, highly educated, scientific members of multiorganizational research and development programs.

Qualitative case study designs are different from other approaches; for example, the environment can be left uncontrolled in a case study design (Yin, 2014). A case study research design provided an option for open-ended learning, consistent with the goal of my study (Yin, 2014). A case study research design allows researchers to explore the decision processes in the real-world context in which they occur (Yin, 2014). This realworld context was important to consider when exploring virtual leadership strategies in the multiorganizational scientific collaboration program.

I selected a qualitative method for this research because leader training expectations did not exist, nor had selection criteria been developed for leaders of multiorganizational scientific collaborations (Vessey et al., 2014). Some research has included examinations of this cross-section of the research and development community, resulting in institutionally complex programs (Jay, 2013) with highly educated participants (Paulsen et al., 2013). New research on the combined effect of the 
organizational structure along with scientific culture adds to the body of knowledge for organizational research.

To understand the elements of case study research, I looked to three theorists for guidance on how to approach case study research. Merriam (2009), Stake (1995), and Yin (2014) provided similar descriptions of a case study as a bounded system. They agreed the phenomenon of interest existed within the context in which it occured. Merriam and Tisdell (2016) agreed with Yin that a case study approach was appropriate when the population of interest was not separate from the phenomenon of interest. Merriam and Tisdell described a case study as an in-depth analysis of a single system requiring an intensive holistic description of the phenomenon. Yin noted that a case study design gives the researcher the opportunity to investigate in more depth than other designs permit. Merriam and Yin agreed that the bounding conditions of the case defined the unit of analysis.

The unit of analysis for this research was the program, which was made up of multiple organizations collaborating as a single super organization. The unit of observation was the individual from a participating organization. The individuals represent only the single organization from which they originated, so multiple organization sampling was required to gain a variety of perspectives.

This qualitative case study included 15 program member interviews from 10 multiorganizational research and development programs under the DHS. I drew from individuals across multiple programs with a variety of experiences to obtain maximum heterogeneity. Through exploratory semistructured interviews, I developed insights into 
leadership constructs of programs within the DHS Centers of Excellence programs. Each scientific collaboration program was made up of a consortium of university and research organizations. The DHS Centers of Excellence programs collectively included more than 100 U.S. and foreign organizations, with more than 1,000 individual participants between them. These programs were targeted at specific and applied research areas such as terrorism risks, visualizing data, transmittable diseases, and coastal security. Without significant preexisting research in these areas to guide a survey of followers, a case study approach allowed for open-ended interviews as the main method of gathering information about followers' perspectives on leadership.

\section{Role of the Researcher}

The role of the researcher is to design the study, conduct the interviews, transcribe the interviews, code the data, and conduct the analysis. As the researcher, I served as the investigation instrument. I performed the interviews, coding, and data analysis. For this study, I implemented a case study design to gather data on a single overarching research question: What virtual leadership strategies do leaders of interdisciplinary collaborations use to motivate their highly educated scientist's members across organizations? As the instrument, I kept a journal of observations during the interview, coding and analysis phases for identification of personal beliefs. Reviewing these observations aided in gathering insights. I was familiar with multiorganizational research and development programs before embarking on this research. I have close to 25 years of experience in scientific collaborations involving primarily graduate-level researchers. 


\section{Qualitative Method}

This study of virtual leadership comprised of exploratory sets of semistructured interviews. Each scientific collaboration program was made up of a consortium of university and research organizations. The DHS Centers of Excellence programs collectively represent more than 100 organizations, with more than 1,000 individual participants between them. Collectively, this set of programs comprised the case study for this research. Out of the $1,000+$ participants within these programs, 15 individuals agreed to participate in this study. The interviewed participants represented 10 different DHS Centers of Excellence programs.

The qualitative method used to study the research question included the identification of program participants, selection and interviewing program participants, transcribing, and coding and analyzing participants input. Two-cycle coding was used to first identify elements within each interview and then to look for patterns across interviews (see Miles, Huberman, \& Saldana, 2014). The initial code list was prepared from the literature review process and represented terminology from previous research. There are three ways to communicate coding in research: narrative descriptions, matrix display, and network displays (Miles et al., 2014). For my research I used all three methods to some degree to explore and communicate observations. These tools were used to help group and review interview content during the second cycle codes analysis.

To allow for holistic analysis, I was the only researcher conducting the interviews and serving as the instrument of analysis. Coding for investigation techniques included methods such as clustering, counting, and building a relationship matrix. I investigated 
outliers for meaning while capturing potential extended areas for future research. Negative evidence appearing to contradict initial findings was a target of data reviews.

This study follows a qualitative case study research design. The literature review consisted of the current literature search, review, and synthesis. The literature review extended to complex system leadership, scientific collaboration, and leader-member exchange theory. The information gathered as part of the literature review informs the development of an initial analysis code list. Program documentation supports characterizing the mission, size, and organization structure. Finally, individual interviews comprised the main data source for my study.

The review of previous literature related to multiorganizational research and development program leadership guided common terminology potentially used by participants. For example, Northouse's (2016) description of identified traits was a starting point for codes interview transcripts. This terminology also served as the basis for the development of the initial semistructured interview questions found in Appendix A.

Documentation on the purpose and history of each program review was part of the program data package. Program documentation aided in understanding the nature and scope of the research at each Centers of Excellence program. Program documentation included elements such as the number of organizations involved, the spatial relationship between organizations, and the scope of projects. I identified gaps and potential areas as starting points for coding and analyzing the results. 
I developed a set of semistructured interview questions following guidelines put forth by Seidman (2013). A draft interview protocol is provided in Appendix A. The resulting questions guided interviews with participants from all programs. A review panel evaluated the interview questions and planned interviewee list before the interviews.

\section{Participant Selection}

All DHS Centers of Excellence programs were the basis for examining a range of experiences for this study. Each program considered had been in existence for more than 1 year. Two programs were new in 2017 and not considered as part of this participant pool. Having completed at least 1 year provided time for leadership processes to be observable by program participants. The publicly available material for a program listing the participating organizations and contacts at each organization could identify participants. Participants were identified through program publications listings and project reports available to the public. Finally, social media such as LinkedIn was also used where appropriate to identify contact information for individuals identified on program publications.

My participant selection process gathered online program information, which included participant names, program size, and the number of organizations. Metrics provided organizational insight using the volume of work, the type of organizations participating, and the number of publications. I focused my study on all available programs. The timeframe for a program lifecycle was variable. Virtual leadership investigation focused on the years the individual programs were operational. Documented material and interviews with multiple participants were part of the program data package. 
The director of each center was offered the opportunity to contribute to the observations for this research.

Individuals included in this study collectively represented both management and research perspectives to facilitate a broader understanding of the multiple viewpoints of leadership. Individual demographic information included the role in the institution represented, the role in the program, education, and research experience. These individual demographics helped determine patterns in relationships between interview content and individual characteristics. The demographics were part of the qualitative instrumentation for this study.

\section{Instrumentation}

The instrumentation for this study consisted of a set of semistructured interview questions with me as the researcher serving as the instrument of analysis. Researchers had previously looked at the transformational leadership component of leader processes (Cole, Bedeian, \& Bruch, 2011). Leadership efficacy from the leader's self-assessment perspective was also previously studied (Hannah, Avolio, Walumbwa, \& Chan, 2012). Previous approaches focused on leader self-assessment rather than the followers' perspectives of the leader. Erdogan and Bauer (2014) suggested future researchers step away from the commonly used seven-question instrument. These researchers recommended using methods to explore the perception of multiple team members demonstrating a relationship with the leader. No available instrument was appropriate for follower perspectives of leadership in virtual scientific collaboration environments. No instrument to evaluate complex multiorganizational research environments was in the 
extant literature. The open-ended nature of this study helps to inform instrument development in the future. The interview questions guided the conversation while gathering participants' perspectives. The information might apply to future instrument development.

The instrumentation for this study was the researcher. As the researcher, I gathered semistructured interview content and conducted the analysis. I captured interviews in the form of audio recordings and then transcribed them. Member-checking was used to share the transcripts back to the participant for review and comment. The interview protocol was designed to answer the following overarching research question: What virtual leadership strategies do leaders of interdisciplinary collaborations use to motivate their highly educated scientists across organizations? Appendix A provides a set of interview questions built to address this research question. Probing subquestions were included to ensure exploratory discussion on noncollocated leaders, interaction with different organizations, and multiple discipline environments.

Additional artifacts, such as documents describing the purpose, structure, and results of each program, were collected as opportunistic information. The sample source for the interviews was participants from programs under the purview of DHS Centers of Excellence programs. These interviews were recorded and transcribed for coding and analysis. The focus of this research was the perception of leadership and leader-member relationships in complex multiorganizational scientific collaboration programs. The interviews program members focused on their perception of virtual leadership strategies. 


\section{Procedures for Recruitment, Participation, and Data Collection}

This qualitative case study research included participant interviews from individuals with experience in multiorganizational research and development programs. The sampling procedure was drawn from individuals with a variety of roles with a goal of achieving a maximally variable sample and heterogeneity. The collective data from 15 participant interviews represented experiences from 10 of the 17 DHS Centers of Excellence programs and provided an overview of perspectives on leadership and motivation.

Recruitment for this research included obtaining participant names, organization, phone numbers, and e-mail address, where available, from the DHS Science and Technology Office of University Programs website for the Centers of Excellence programs (U.S. DHS, 2017). A request for participation e-mail was sent to individual participants identified or associated with a program whose e-mails could be located. The interview protocol used is provided in Appendix A. The Walden University Institutional Review Board approval number for this study was 03-15-17-0175576.

As part of the recruitment process, I e-mailed a consent form to each participant who had expressed an interest in my research. The returned consent form was documentation of their agreement to participate in a semistructured interview. As part of the receipt of consent, each participant received a copy of the interview questions. At the time of consent, I also gathered potential interview times. A Microsoft Lync conference line was scheduled once confirmation was received (Lync, 2015). Instructions for a tollfree number and conference call code were sent to each participant, noting their 
scheduled time and anticipated duration. In cases where the participant did not respond to the first consent form e-mail, a second reminder consent form e-mail was sent. In some cases a third e-mail was sent confirming the interesting in participating.

Each interview was anticipated to take approximately one hour. At the start of the conference call, I asked each participant whether they were still comfortable with recording the interview. If the participant agreed, then the recording device was turned on. Lync notifies all individuals on the call when a recording has started. After starting the recording, I confirmed again that the participant understood the purpose of the interview and was still comfortable taking part in the recorded discussion. I confirmed the receipt of the interview questions, and then asked whether they were any questions before beginning the interview. Appendix A contains a more detailed description of the protocol.

I conducted the interviews with participants. The data collection approach included audio recordings using the Lync teleconferencing tools (Lync, 2015) and notes taken by me during the interview. An audio recording of each interview was an artifact of my study and stored in the package of evidence. The end use of this data stream was to provide quality transcripts and associated coding. Transparency in coding yielded more comprehensive insights into virtual leadership in complex multiorganizational scientific collaboration environments.

Data collection included interviews and organizational documentation. Data saturation was a current topic among qualitative researchers (Hennink, Kaiser, \& Marconi, 2017; Malterud, Siersma, \& Guassora, 2016). Saturation was achieved when no new themes, perspectives or insights were evident when additional data was gathered and 
reviewed (Patton, 2015). According to Fusch and Ness (2015, p. 1408), “data saturation is reached when there is enough information to replicate the study, when the ability to obtain additional new information has been attained, and when further coding is no longer feasible." The subjective nature of qualitative data gathering and review process lends itself to multiple forms of interpretation. Much like qualitative study itself, the researcher played a large role in defining and defending the evidence for saturation. Factors playing into the determination of saturation were; the purpose of the study, research design, characteristics of the population being studied, analytic approach to analysis, and available resources (Hennick et al., 2017). The purpose of my qualitative investigation was to gain an understanding of followers' perspectives regarding virtual leadership within complex multiorganizational programs. My research design was a qualitative case study. My population of interest was participants in multiorganizational scientific collaboration programs made up of highly educated individuals. My analytic approach used a case study design with an iterative analysis methodology to guide findings and identify saturation.

All references to names of organizations and people were coded in the transcript avoiding connecting specific statements to individuals and organizations. Opportunistic interviews helped to collect as much information as possible for analysis.

The Common Operating and Response Environment (CORE) data collection system was used to store all organization information and demographics summaries, pre-interview information for potential participants, and reminders (PNNL, 2015). After each interview, all recordings were stored separately, while coded transcripts and comments were 
uploaded into the CORE data sheet to preserve the package of evidence necessary for defensible data collection. I transcribed each audio recording of interviews. I used member-checking to provide participants the opportunity to comment on transcriptions of their interview and initial coding sets. The participant's package of evidence was stored in the CORE data collection system as a text file containing the transcription and tagged with the associated codes. The personally identifiable information for each participant and their associated code was stored separately. Figure 2 depicts the elements of the participant package of evidence stored in the data collection system.

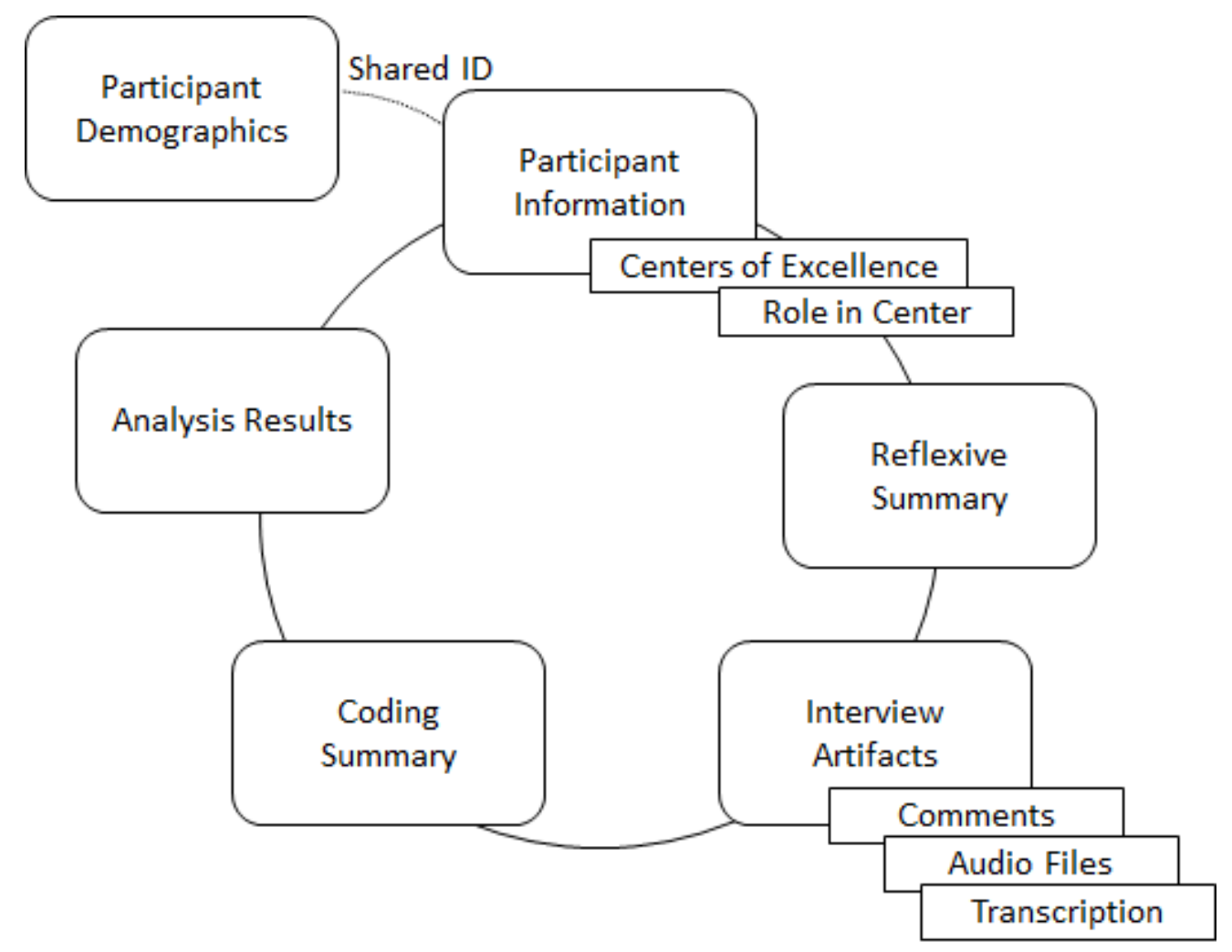

Figure 2. Package of evidence for each interview participant.

The package of evidence for my research consists of five elements; (a) participant summary information, (b) reflexive summary from the researcher following the 
interview, (c) interview artifacts, (d) coding summary, and (e) analysis results. I kept the participant's demographics in a spreadsheet separate from the online package of evidence. Only the participant's ID appeared online as part of the package of evidence. An additional data collection activity included in the package, was to gather any documentation and materials related to leadership, program organization, and processes. Documentation of all information gathered was stored in the CORE technology document library. The CORE library made each of the documents referenceable, with comments, tags, and full text searchable.

\section{Data Analysis Plan}

I sent a request for participation to each potential participant identified as part of the program. Each participant who expressed an interest in contributing were sent a set of interview questions. I transcribed and coded interview recordings. I categorized the interview results to provide an overview of leadership in complex multiorganizational research and development programs. I highlighted the processes, leadership challenges, and constructs to manage complex multiple team systems from the follower's perspective. Associating each interview question with a specific mission area in the Centers of Excellence program enabled me to learn how staff fostered creativity, crossed organizational boundaries, encouraged scientific collaboration, developed interdisciplinary teams, and provided customer-driven solutions. Using the participants' semistructured interviews for this study allowed me to align interview responses across cases. 
Additional data for review during analysis included a short memo capturing my immediate observations and thoughts regarding each interview. Memo's allowed me to look for additional insights to be captured during the interviews regarding themes and observations at multiple points in the research process. If I added a new theme, I wrote a short memo to capture my reasoning and assumptions for the inclusion of the additional coding. Some recurring themes emerged before all of the interviews were conducted. All participants positively responding to the request to participate were interviewed regardless of potential saturation before the final interviews were completed.

Fusch and Ness (2015) suggested a methodology requiring an iterative analysis of information collection. In my case, these were individual semistructured interviews. The guidance provided by Fusch and Ness suggested coding of interviews as they were collected in order to track new codes as they emerged. This information fits well with Hennink et al. (2017) emergent view of coding themes.

Hennink et al. (2017) provided examples of two approaches to defining and defending saturation constructed on codes emergence and code meaning. Their example dataset was semistructured interviews which were consistent with my research. Hennink, et al. code theme approach provided a visualization of code emergence and stabilization. It highlighted when a code theme emerged as well as when it stabilized as the data collection progressed over time. The time factor of their work supports the idea that there was more than one dimension to coding.

Hennink, et al. (2017) also recommended code meaning being assessed in addition to code frequency. Looking at the value of the code and its purpose in the larger 
analysis has the potential to help identify saturation. This was consistent with what Malterud et al. (2016) referred to as the strength of the dialogue. Fusch and Ness (2015) also discussed the need for quality data and used the term 'rich' to indicate depth and the term 'thick' to represent the quantity of information.

Malterud, et al. (2016) suggested that determination of saturation should be calculated using an information power equation. Nelson (2016) had a similar approached through using conceptual depth criteria. The information power for a sample was determined by study aim, sample specificity, use of established theory, quality of dialogue, and analysis strategy (Malterud et al., 2016). Each of these measures provided a way to look at the information from a different angle and determine how good, or bad the data might be.

Malterud et al. (2016) measured study aim by how broad or narrow it was. The aim of my research was to provide some insights into the virtual leadership of multiorganizational scientific collaboration programs. Ultimately, this work could improve the likelihood of future investments in government-funded research and development efforts resulting in innovative behaviors leading to innovative solutions to challenging problems. My research aim fell into Malterud et al. description of a broad range. This indicated a larger sample size was needed to meet saturation. Malterud, et al. sample specificity was measured as either dense or sparse. Specificity was a way to look at the homogeneity of the population. My study was looking at highly educated participants of scientific collaboration programs. My population has many similar 
characteristics indicating a smaller sample size would be required for saturation (Malterud et al., 2016).

Several researchers have indicated the need for thick dialog when conducting interviews (Fusch \& Ness, 2015; Malterud et al., 2016; Patton, 2015). Malterud, et al. (2016) referred to this as the quality of the dialog and scored it as either weak or strong. Fusch and Ness (2015) discussed interviews in terms of rich to represent quality and think to represent quantity. My research interviews were predominantly strong in-depth dialog. This along with the thick interview dialog scored my research as strong in quality of dialog. According to Malterud et al., a strong dialog would require fewer interview sessions than a week dialog.

The quality of the dialogue was also impacted by the depth of understanding the researcher had regarding the subject being discussed (Fusch \& Ness, 2015; Malterud et al., 2016; Stake, 1995; Yin, 2014). Stake's (2006) expectation on the appropriate use of case study research was dependent on the researcher's expertise in interviewing, analyzing, and revisiting research processes throughout the duration of the case study. Understanding the point of completion and the study goals well enough to remain on track during this iterative process was daunting and may not be possible for researchers new to the method (Stake, 1995). Yin's (2014) structured methodology provided a basis for planning and conducting case study research. One of Yin's strengths was providing the researcher a variety of methods for collecting and organizing data to aid in the systematic review and reporting of findings. Within my own research, I am very familiar 
with collaborative scientific environments, working with highly educated individuals in an academic environment, and working virtually with colleagues.

Malterud et al. (2016) provided a final category, analysis strategy. Their strategy was separated into a single and multiple case approaches. Single case studies required less data than multiple case studies (Multerude et al., 2016). This was intuitive because a multiple case study approach would require enough data within any one case study to conduct an independent analysis. Each subsequent case study would also require a sufficient dataset. A single case study required only one set of participants to be aligned before saturation was achieved. I implemented a single case study design with a goal of evaluating saturation across multiple program participants.

Coding was conducted to identify themes such as leadership terminology, trust, and communication. Common themes emerged and were assigned a tag included in the coding. A two-cycle coding approach was applied (Miles et al, 2014). Descriptive and process coding were most applicable to this study. The combination of these codes allowed for exploration of program leadership processes and attitudes regarding leadership. Coding drawn from the literature review included material regarding scientific collaboration, leader-member exchange, and complex systems theories. Codes and themes were derived from analysis and visualization of the information.

Visual displays of information was a growing method of scientific investigation (Krallinger et al., 2017; Kwon, Kim, \& Park, 2017; Wiedemann, 2016). Visualization allows the human mind to see connections and patterns faster than looking at numbers or words (Niemann, Moehrle, \& Frischkorn, 2017). Text analysis and visualization was a 
natural fit for social science and qualitative explorations (Wiedemann, 2013). Wedemann (2016), noted that for qualitative research specific analysis requirements and data management planning were critical to the successful implementation of valid and reliable computer-assisted text analysis. His work took a holistic view of automated text analysis tools and techniques and a perspective for their potential use in social science with qualitative analysis expectations. My transcript dataset was not large, yet exploratory use of a semantic analysis and visualization tool allowed for investigation from a variety of coding perspectives while providing visual images of the resulting patterns and text clustering (Erkens, Bodemer, \& Hoppe, 2016).

Tools have emerged in recent years for semiautomatic exploration of text-based datasets. TextTile (Felix, Pandey, \& Bertini, 2017), ThemeRiver (Havre, Hetzler, \& Nowell, 2000), TextFlows (Perovšek, Kranjc, Erjavec, Cestnik, \& Lavrač, 2016), and INSPIRE (Potel, \& Wong, 2014) were examples of tools allowing researchers to probe text for patterns, relationships, and content. Each of these tools used statistics and visualization to aid the human analyst in understanding the content of documents. Each tool took a slightly different approach for data interaction and visualization.

TextTile (Felix et al., 2017) took a combined approach for looking at data and unstructured text in combination with a data visualization tool for exploring data sets and questions requiring seamless analysis. TextTile researchers based their development on real-world challenges in data analysis. TextTile incorporated a set of interchangeable operations using both structured and unstructured text information producing common data summaries. Their summaries used visual tiles in a grid layout to organize analysis 
and associations. TextTile could not be used in my research because my dataset consists only of unstructured text. There was no associated structured dataset to improve the evaluation of the information.

ThemeRiver (Havre et al., 2000) focused visualization of unstructured text over time. Their interface was designed to help users identify time-related patterns, trends, and relationships across a large collection of documents. ThemeRiver used a river analogy to support the image of the analysis as represented by a flow from left to right through representing time and text themes. The graphic narrowed or widened to indicate a change in the collective impact of themes in the underlying documents. Individual themes were represented as colored river currents flowing. The currents' change in size depicted a change in individual theme strength at any point in time. ThemeRiver depends on a set of documents crossing over a particular timeframe. The dependency on time-phased unstructured text in order for ThemeRiver to provide insightful results; it was not selected for my research. The interviews were conducted over a period of months, but the time of the interview was not expected to be an indicator as it relates to patterns emerging.

TextFlows (Perovšek et al., 2016) provided an open-source online platform for composition, execution, and sharing of interactive text mining and natural language processing workflows. TextFlows provided a graphical user interface for developing workflows as building blocks. The blocks provide a simplified representation of complex procedures into a spatial for users to begin their analysis. TextFlows was not an option for my research because of the private nature of the interviews collected. The information 
for my research cannot be posted to the web cloud for analysis in the event any of the information in the text-based discussions could be used without permission.

Data analysis was conducted using tagging and coding methods. Several rounds of coding were conducted to identify themes. Four software packages served to capture, store, and analyze research artifacts: (a) Microsoft Lync for conducting and recording interviews; (b) CORE for storing, organizing and tagging transcripts, memos, and reference materials used; (c) Microsoft Office Visio was used for documenting processes through flow diagrams and relationship charts; and (d) Microsoft Excel was used to capture and visualize program specific information, demographics, and participant response summaries.

In addition to interview coding, documentation of each program was gathered to provide demographics at a program level. These documents were used to add to the interview coding list and to support/negate emerging observations. Results included highlighting similarities, differences, and patterns answer the following research question: What virtual leadership strategies do leaders of interdisciplinary collaborations use to motivate their highly educated scientists across organizations?

\section{Issues of Trustworthiness}

\section{Credibility}

The first critical step in applying a quality discussion to research was to provide clarification on the definition of quality in this context. For this research, I defined quality as transparency in the process with an insightful evaluation of the information gathered. It was important to document each step in the process with comments to provide 
traceability. Data management, member-checking, and peer reviews were used to ensure a credible approach and results. The first aspect of quality was in the management of raw data and commentary. It was imperative that sufficient and reliable audio equipment was used to preserve the interviews.

An important aspect of quality related to credibility involved the accuracy of interview transcription and interpretation through two forms of member checking. I transcribed the interviews with initial coding applied. The interview transcript and the initial coding applied were both returned to the participant to ensure the accuracy of the transcript as well as the accuracy of observations. These two types of member-checking allowed the participants, if they were interested, to fully engage in the quality and interpretation of their contribution before the coding process was completed. Also, the data and findings were sufficiently annotated, so publishing results in peer-reviewed publications was an option. Finally, to ensuring data collection quality, all information was maintained in a secure electronic filing system so my research processes could be repeated.

To maximize trustworthiness of the results, a process was developed and followed which included data gathering, analysis and reviews. Figure 3 provides an overview of the processes put in place to systematically explore the information gathered. The code development was derived from both listening to the interview and reviewing the transcription of the interview. This information, along with feedback through memberchecking came together in the coding review for each individual. After each individual was coded, the participant's codes were put in order of date interviewed. This 
information was reviewed for common themes, and emergent themes were documented.

Additional information was gathered from the program websites to enhance the findings.

Finally, data visualizations were used to confirm findings across all participants

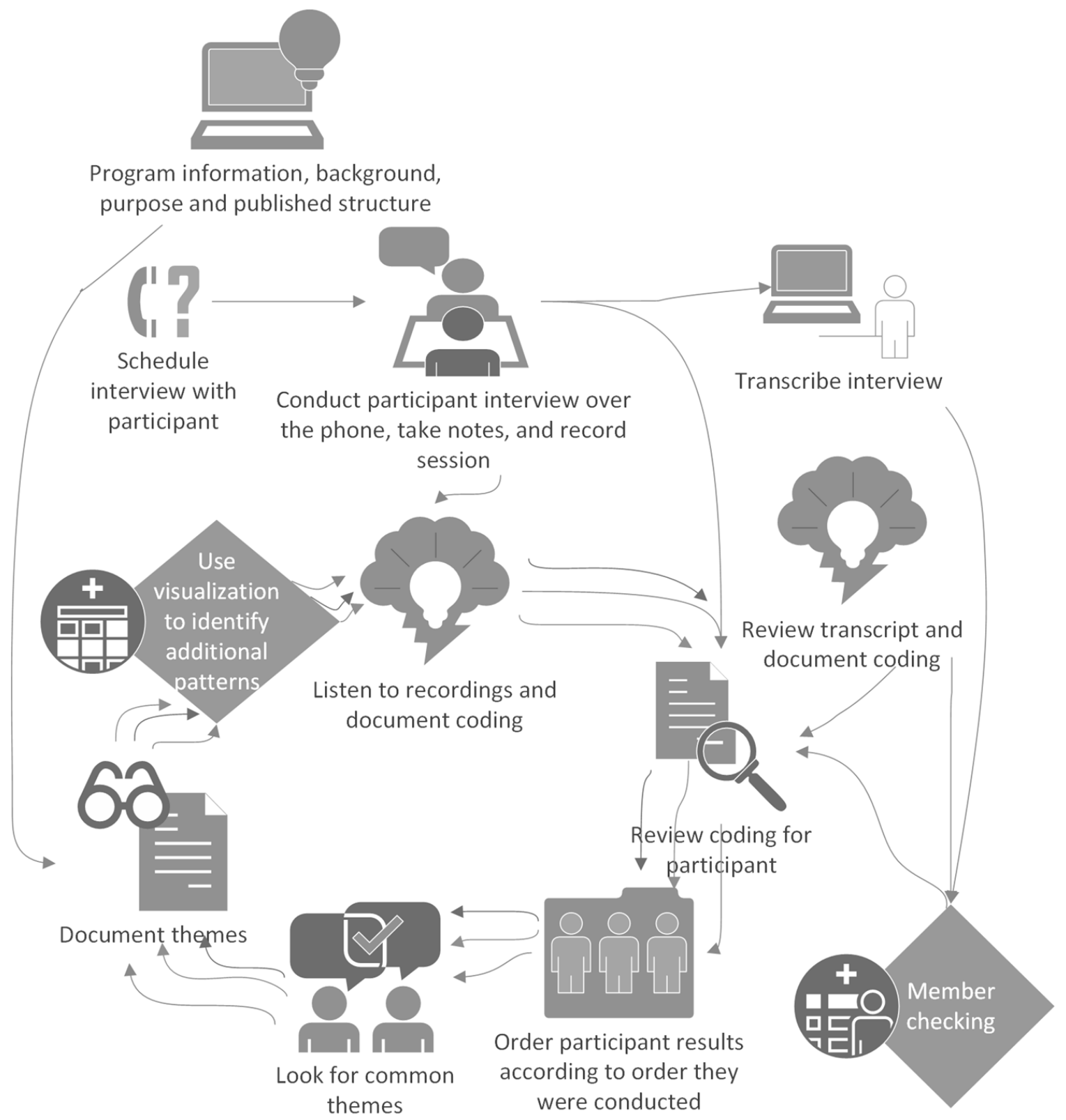

Figure 3. Code development and analysis process. 
My exploration processes included multiple rounds of coding, exploring, and summarizing information. The code cycle was conducted in three rounds. Even though themes were apparent after the second round of analysis, further exploration was conducted to incorporate additional interview input and confirmation of consistencies.

\section{Transferability}

Transferability for my researcher was an indication of the external validity of my findings. Miles et al. (2014) recommended clarity in information regarding the research as the best way to ensure optimal transferability. For this case study, detailed descriptions of the original sample, setting, processes, and analysis techniques were provided in this report to increased potential for transferability. Additional information on the formation, background, purpose, and structure of each program were included to allow future researchers to draw potential parallels to this study. Transferability increased by the thick descriptions from the participants captured in the interviews. Finally, results found to be congruent with previous research was noted.

\section{Dependability}

Several review interview questions covered similar target topics. These openended questions were used to avoid potential issues with quality and dependability. These questions were designed to ensure the participant pool was diverse and representative. Questions regarding participant's role(s) were used to analyze the participant pool to maximize heterogeneity. Findings were critically reviewed for categories of interest and discrepancies. I used questions about the conditions under which the emerging theme(s) 
hold up to ensure the concepts relate to the findings. These multiple methods together helped establish the validity of the findings.

\section{Confirmability}

Because I served as the instrument of analysis for this study, I implemented several activities for identifying and reporting bias. I used journaling to capture my initial and changing perspectives. I also collected member's input through interview questions. I used the process of transcription, coding, and pattern identification to verify logic and defensibility. Data and findings reviews were iterative to allow for updates to coding and revisiting any patterns identified.

\section{Ethical Procedures}

A communication strategy was defined here for how, what, and when potential participants were engaged. I included the consent form in the Lync (2015) meeting e-mail sent to each participant. Participants who did not want to continue were removed from the participant list. Consent included informing the participants of how I planned to use the findings. I included an explanation of the interview recording approach. I ensured each participant received and read the informed consent material, through requesting the concent be returned via e-mail before the interview was scheduled. The follow-up consent e-mail also gave the participant an opportunity to ask clarifying questions. The follow-up consent e-mail ensured the participant was interested in continuing in the study. Participants reviewed the anticipated process again during the actual interview. In the interviews, I confirmed again with the participant their comfortable with recording the interview. The Lync software also notified listeners when a recording started. After 
the recording had begun, I repeated the planned process and received a second verbal agreement from the participant before conducting the actual interview. In this way, I captured the communication and acknowledgment for authorization to record as part of the session recording. All participants opted to continue with the interview and remain a part of my study.

\section{Summary}

I have selected a qualitative methodology with a case study design to conduct a study on the virtual leadership of multiorganizational research and development programs. I captured data through interviews with participants across multiple programs. The participants were drawn from members of DHS Centers of Excellence programs as the participant pool. My study included targeted interviews with a variety of individuals representing a diverse array of perspectives. In this study, I sought to answer the question: What virtual leadership strategies do leaders of interdisciplinary collaborations use to motivate their highly educated scientists across organizations?

This study enhanced my understanding of leadership processes in complex multiorganizational research and development programs focused on solutions for significant global challenges. Results of my case study provided a rich representation of diverse leadership observations from the followers' perspectives. This study makes an important theoretical contribution to multiorganizational program leadership theory by addressing the gap in current research. I used a scientific collaboration lens to explore the gap between complex systems leadership and multiteam systems research. This study enhances emerging research in complex system leadership. Information on 
interdisciplinary teams operating in distributed environments was shone to be of interest when taking into account collaborative scientific culture.

I provided a set of interview questions to the participants who expressed an interest in my study. I transcribed and coded interview recordings. The interview results were categorized to provide an overview of leadership in multiorganizational scientific collaboration programs, such as the DHS Centers of Excellence programs. In my findings, I highlighted the processes, leadership challenges, and constructs to manage complex multiple team systems from the follower's perspective. I recruited a list of individuals from across all Centers of Excellence programs through identifying them on their program's official website and related links. Individuals expressing an interest in the research received the interview questions with a consent form containing information on the content and duration of the interviews.

Interviews were recorded, transcribed, and coded in preparation for analysis. I collected analysis of patterns, similarities, and differences in leadership strategies as they related to the multiorganizational nature of the programs operating within virtual environments. Additionally, I Assessment of outliers and critical reviews seeking negating evidence provided an opportunity to identify possible alternative reasoning. I posted all of the data and documentation on a secure web server as a package of evidence for review. Specific participants were coded, and the code list of participants' names was stored in a separate location as the results. I used member-checking by providing the participant's transcripts and initial coding. This coding represented my thoughts as the 
instrument for this research. A history of changes and any additional input provided by the participant was part of the package of evidence for this research.

Understanding the leadership in the scientific collaboration environment in the multiorganizational research and development programs was critical for my study of virtual leadership. Research on in this area informs leadership models. Considerations for models provided could support additional theory development to target opportunities for positive change in this scientific research environment training expectations. My study could increase the likelihood of such programs producing novel solutions to globally challenging problems of water resource management, climate change, healthcare delivery, and national security. 
Chapter 4: Results

The overarching research question of this dissertation was: What virtual leadership strategies do leaders of interdisciplinary collaborations use to motivate their highly educated scientists across organizations? To address the research question, I conducted a qualitative case study of the participants in the DHS Centers of Excellence programs to understand virtual leadership within complex multiorganizational scientific collaboration programs from the perspective of program participants or followers. I characterized successful leadership of complex multiorganizational scientific collaboration programs as fostering creativity, crossing organizational boundaries, encouraging interdisciplinary collaboration, and providing customer-driven solutions. These characteristics were goals for the DHS Centers of Excellence programs (U.S. DHS, 2017). The focus on program participants' perspectives regarding virtual leadership and interdisciplinary collaboration addresses an important research gap in the research on virtual leadership in multiorganizational scientific collaborative environments. Previous research emphasized the organizational leaders' perspective of virtual leadership via selfassessment. There has been an increasing need within the leadership research community to incorporate a more holistic consideration of leadership of complex multiorganizational systems (Dinh et al., 2014; Henry, 2015). Additionally, understanding the context of the program team dynamics in interdisciplinary collaborations of highly educated individuals was necessary (Vessey et al., 2014). In this section, I describe the research setting, sampling method, data collection and coding procedure, and research findings. 


\section{Research Setting}

A case study research design facilitated exploration of complex multiorganizational scientific collaboration programs focused on creating solutions for national and global challenges. The focus of my study was to understand virtual leadership strategies of interdisciplinary collaborations represented by DHS Centers of Excellence programs. The DHS Office of University Programs oversees the Centers of Excellence programs. Each program was assigned a program manager within the Office of University Programs. Each program manager may have one or more programs to guide and ensure expectations were being met. One, or in some cases two, universities were identified as the lead organization a particular program. In most cases this was a single university; however, in scenarios where there were two universities, the universities operated as two mini-programs rather than one fully integrated program between the two university leads. An example of this was the Center for Visualization and Data Analytics (CVADA). Their work was led by Purdue University through their Visual Analytics for Command, Control, and Interoperability Environments (VACCINE), and the second university, Rutgers University, led a second branch of the program through their Command, Control, and Interoperability Center for Advanced Data Analysis

\section{(CCICADA).}

The DHS Office of University Program's charter was designated to commission universities and coordinated organizations to collectively apply novel thinking to problems related to national security. The choice of universities as leaders was to fulfill the goal to help train the next generation of homeland security experts. The DHS Centers 
of Excellence programs worked closely with academia, industry, first responders, and DHS operational components such as Coastguard, Customs and Border Protection, and the Transportation Security Administration (U.S. DHS, 2017). These programs were expected to develop customer-driven, innovative tools and technologies to solve realworld challenges. Each program targeted national security technical areas focused on real-world challenges. In this way, programs were designed to bridge academic environments and applied settings. This bridging expectation in itself adds to the complexities faced by DHS Centers of Excellence leaders.

Four Centers of Excellence programs were initially started in 2004. Over time new programs were started. Programs that were no longer a priority, or ineffective, were ended. Two of the current DHS Centers of Excellence programs were new in 2017 and were not included in this study. My study was limited to programs in place for more than 1 year. Limiting the timeframe to the initiation of my research enabled me to gather participants' experiences within the programs over time. Table 1 provides a program name and focus area for the two new DHS Centers of Excellence programs. The new programs were started in 2017 and had yet to complete their first year of operation. These two new programs were not included in this study because they did not meet the criteria of being in operation for at least 1 year prior to my study beginning. 
Table 1

New 2017 DHS Centers of Excellence Programs and Focus Areas

\begin{tabular}{ccl}
\hline Program & Name & \multicolumn{1}{c}{ Program science and technology focus area } \\
\hline \multirow{2}{*}{ CAOE } & $\begin{array}{c}\text { Center for } \\
\text { Accelerating } \\
\text { Operational } \\
\text { Efficiency }\end{array}$ & $\begin{array}{l}\text { CAOE will apply advanced analytical tools to optimize } \\
\text { efficiency in homeland security operations. } \\
\text { (CAOE, 2017) }\end{array}$ \\
& $\begin{array}{c}\text { Criminal } \\
\text { CINA }\end{array}$ & $\begin{array}{l}\text { CINA will develop strategies and solutions to enhance } \\
\text { criminal network analysis, forensics, and investigative } \\
\text { Investigations and } \\
\text { processes for on-the-ground use by agents and officers } \\
\text { to predict, thwart, and prosecute crimes (CINA, 2017). }\end{array}$ \\
\hline
\end{tabular}

Table 2 lists the continuing programs. Current programs were those which continue to be actively supported by DHS through 2017 . These programs receive a baseline of funding to operate the program in addition to funds for supporting research. Most of these current programs have been active for three years. START is an outlier, having been active for more than the 12-year life-cycle expected for Centers of Excellence. Current programs have updated materials available on the DHS Office of University Programs website as they continued to conduct research.

Three of the current programs are related to former programs under DHS. The Coastal Resilience Centers of Excellence program included a complementary perspective of resilience rather than hazards prediction, which was the vision for its predecessor the Coastal Hazards Centers of Excellence program. The Borders, Trade, and Immigration Institute program had a similar vision to the National Center for Border Security and Immigration former program. The inclusion of trade into the vision for this program distinguished it from its predecessor. Finally, the Maritime Security Center program had a similar vision to the Maritime, Island and Remote and Extreme Environment Security 
program. The extension to other forms of maritime was a distinction within the current program.

\section{Table 2}

Current DHS Centers of Excellence Program Focus Areas

\begin{tabular}{|c|c|c|}
\hline Program & Name & Program science and technology focus area \\
\hline $\mathrm{ADAC}$ & $\begin{array}{l}\text { Arctic Domain } \\
\text { Awareness Centers of } \\
\text { Excellence }\end{array}$ & $\begin{array}{l}\text { ADAC improved situational awareness and crisis response } \\
\text { capabilities related to emerging maritime challenges posed by the } \\
\text { dynamic Arctic environment (ADAC, 2017). }\end{array}$ \\
\hline ALERT & $\begin{array}{l}\text { Centers of Excellence } \\
\text { for Awareness \& } \\
\text { Localization of } \\
\text { Explosives-Related } \\
\text { Threats }\end{array}$ & $\begin{array}{l}\text { ALERT conducted research to characterize, detect, mitigate, and } \\
\text { respond to explosives-related threats facing the country and the } \\
\text { world. (ALERT, 2017) }\end{array}$ \\
\hline BTI & $\begin{array}{l}\text { Borders, Trade, and } \\
\text { Immigration Institute }\end{array}$ & $\begin{array}{l}\text { BTI researched to enhance the Nation's ability to secure the } \\
\text { borders, facilitate legitimate trade and travel, and ensure the } \\
\text { integrity of the immigration system (BTI, 2017). }\end{array}$ \\
\hline $\mathrm{CRC}$ & $\begin{array}{l}\text { Coastal Resilience } \\
\text { Centers of Excellence }\end{array}$ & $\begin{array}{l}\text { CRC addressed topics related to building resilience in coastal } \\
\text { communities (CRC, 2017). }\end{array}$ \\
\hline MSC & $\begin{array}{l}\text { Maritime Security } \\
\text { Center }\end{array}$ & $\begin{array}{l}\text { MSC targeted maritime domain awareness and developed } \\
\text { strategies to support marine transportation system resilience (MSC, } \\
\text { 2017) }\end{array}$ \\
\hline START & $\begin{array}{l}\text { National Consortium } \\
\text { for the Study of } \\
\text { Terrorism and } \\
\text { Responses to } \\
\text { Terrorism }\end{array}$ & $\begin{array}{l}\text { START focused on the scientific study of the causes and human } \\
\text { consequences of terrorism in the United States and around the } \\
\text { world (START, 2017) }\end{array}$ \\
\hline
\end{tabular}

Table 3 provides the program name and focus area for each emeritus program. Emeritus programs were those supported by DHS in the past and remain functioning programs through alternative funding sources. These programs usually retain a contract through DHS allowing them to request work and transfer funds more quickly than would otherwise be the case. 
Table 3

Emeritus DHS Centers of Excellence Program Focus Areas

\begin{tabular}{|c|c|c|}
\hline Program & Name & Program science and technology focus area \\
\hline CREATE & $\begin{array}{l}\text { National Center for } \\
\text { Risk and Economic } \\
\text { Analysis of } \\
\text { Terrorism Events }\end{array}$ & $\begin{array}{l}\text { CREATE's mission was to improve our Nation's security through } \\
\text { the development of advanced models and tools for the evaluation } \\
\text { of the risks, costs and consequences of terrorism and to guide } \\
\text { economically viable investments in homeland security (CREATE, } \\
\text { 2017). }\end{array}$ \\
\hline CVADA & $\begin{array}{l}\text { Center for } \\
\text { Visualization and } \\
\text { Data Analytics }\end{array}$ & $\begin{array}{l}\text { CVADA was two sub-programs managed separately. Command, } \\
\text { Control, and Interoperability Center for Advanced Data Analysis } \\
\text { (CCICADA) and Visual Analytics for Command, Control and } \\
\text { Interoperability Environments (VACCINE) were managed } \\
\text { collectively to address visual and data analytics to enable swiftly } \\
\text { sifting through a large sets of information, in diverse forms, to get } \\
\text { early warning of potential threats (CVADA, 2017). }\end{array}$ \\
\hline FPDI & $\begin{array}{l}\text { Food Protection and } \\
\text { Defense Institute }\end{array}$ & $\begin{array}{l}\text { FPDI sought help make the nation's food system less vulnerable to } \\
\text { a biological or chemical attack (FPDI, 2017). }\end{array}$ \\
\hline ZADD & $\begin{array}{l}\text { Centers of } \\
\text { Excellence for } \\
\text { Zoonotic and } \\
\text { Animal Disease } \\
\text { Defense }\end{array}$ & $\begin{array}{l}\text { ZADD addressed protecting the nation's agriculture and public } \\
\text { health sectors against high-consequence transboundary, emerging, } \\
\text { and zoonotic disease threats (ZADD, 2017). }\end{array}$ \\
\hline
\end{tabular}

Table 4 lists the former Centers of Excellence programs and their focus areas.

These programs were initiated by DHS but have since been disbanded. Former programs no longer have a contract in place with DHS to support occasional project work. Former programs may even be removed from DHS's Centers of Excellence website, such as the Coastal Hazards Centers of Excellence program (2017). 
Table 4

Former DHS Centers of Excellence Program Focus Areas

\begin{tabular}{|c|c|c|}
\hline Program & Name & Program science and technology focus area \\
\hline CAMRA & $\begin{array}{l}\text { Center for } \\
\text { Advancing } \\
\text { Microbial Risk } \\
\text { Assessment }\end{array}$ & $\begin{array}{l}\text { CAMRA focused research towards preparing and providing } \\
\text { the best tools for decision and policy makers to mitigate } \\
\text { microbial hazards (CAMRA, 2017). }\end{array}$ \\
\hline $\mathrm{CHC}$ & $\begin{array}{l}\text { Coastal Hazards } \\
\text { Centers of } \\
\text { Excellence }\end{array}$ & $\begin{array}{l}\text { CHC conducted research and education to advance the } \\
\text { understanding of natural hazards and community resilience } \\
\text { and transfers that knowledge into action, resulting in } \\
\text { reduced loss of life and less damage to homes, businesses, } \\
\text { infrastructure, and the natural environment (CHC, 2017) }\end{array}$ \\
\hline CIRI & $\begin{array}{l}\text { Critical } \\
\text { Infrastructure } \\
\text { Resilience } \\
\text { Institute } \\
\text { Center for }\end{array}$ & $\begin{array}{l}\text { CIRI was addressing resiliency of the Nation's critical } \\
\text { infrastructures, and the businesses and public entities that } \\
\text { own and operate those assets and systems (CIRI, 2017). }\end{array}$ \\
\hline MIREES & $\begin{array}{l}\text { Center for } \\
\text { Maritime, Island } \\
\text { and Remote and } \\
\text { Extreme } \\
\text { Environment } \\
\text { Security }\end{array}$ & $\begin{array}{l}\text { MIREES sought to strengthen maritime domain awareness } \\
\text { and safeguard populations and properties unique to U.S. } \\
\text { islands, ports, and remote and extreme environments } \\
\text { (MIREES, 2017). }\end{array}$ \\
\hline NCBSI & $\begin{array}{l}\text { National Center } \\
\text { for Border } \\
\text { Security and } \\
\text { Immigration }\end{array}$ & $\begin{array}{l}\text { NCBSI sought to protect the nation's borders from terrorists } \\
\text { and criminals, ease international trade and travel, and } \\
\text { provide a deeper understanding of the forces that lead } \\
\text { foreigners to try to immigrate (NCBSI, 2017). }\end{array}$ \\
\hline NTSCOE & $\begin{array}{l}\text { National } \\
\text { Transportation } \\
\text { Security Centers } \\
\text { of Excellence }\end{array}$ & $\begin{array}{l}\text { NTSCOE addressed all aspects of transportation security } \\
\text { including identification of existing and emerging threat, } \\
\text { development of new technologies for resilient infrastructure, } \\
\text { the establishment of national transportation security policies } \\
\text { (NTSCOE, 2017) }\end{array}$ \\
\hline PACER & $\begin{array}{l}\text { Centers of } \\
\text { Excellence for } \\
\text { Study of } \\
\text { Preparedness and } \\
\text { Catastrophic Event } \\
\text { Response }\end{array}$ & $\begin{array}{l}\text { PACER focused on improving the nation's preparedness and } \\
\text { ability to respond to disasters through rigorous scientific } \\
\text { research focused on medical and public health preparedness } \\
\text { strategies, response capabilities, and surge capacity } \\
\text { (PACER, 2017) }\end{array}$ \\
\hline
\end{tabular}


My study population spanned all programs which existed before 2017, including current, emeritus, and former programs. The 15 participants interviewed for my study shared experiences in current, emeritus, and former programs. I explored the demographics of my research population from a variety of angles. In this section I report the demographic characteristics of the program population regarding the number of affiliated organizations, program duration, and the count of publications, and number of participants within a program. At the program level, duration of operation, number of projects, participating organizations, and number of publications were collected to characterize the participant pool beyond the participants themselves. Program duration refers to the number of years a program was active. The number of projects refers to the number of distinctive technical research areas identified as participating in the program and highlighted in the material. A number of organizations included the listing of organizations identified in the program material as being a partner organization. In some cases these organizations were not called out in the program material provided online; for those programs, organizations were identified through researching program publications for associated organizations of authors. The number of organizations associated with the program could indicate a level of complexity in program structure. The number of publications associated with the program might be a useful indication of the level of productivity, impact or prestige of the program. To that end, I collected data regarding the number of publications listed on the program's website. Collectively across the 17 DHS Centers of Excellence programs represented more than 100 years combined operation, over 300 organizations participating, and over 1,000 publications. 
The count of organizations participating in the DHS Centers of Excellence program was identified by summing the number of unique organizations related to the participant listing. I collected the organizational listings from program materials provided by DHS (2017). The participant listing was from information on the specific programs and publications, and in some cases, the program offered a listing of members. Most programs' websites provided a listing of organizations participating in research, in some cases those organizations did not match the organization identified when looking at the specific program participants lists. For this reason, the number of participating organization included in the demographic discussion was the number of organizations associated with the potential participant list collected as part of the sampling for this research. Figure 4 depicts the number of organizations identified within each program. Most of the programs had less than 30 organizations identified, though one significant outlier had almost 70 organizations identified within the single program. The mean of the number of programs was 20 organizations, with a median of 26 and a mode of 22 . Most programs had between five and 25 organizations. There was an outlier in one of the programs with almost 70. Likewise, several of the programs had less than 10. 


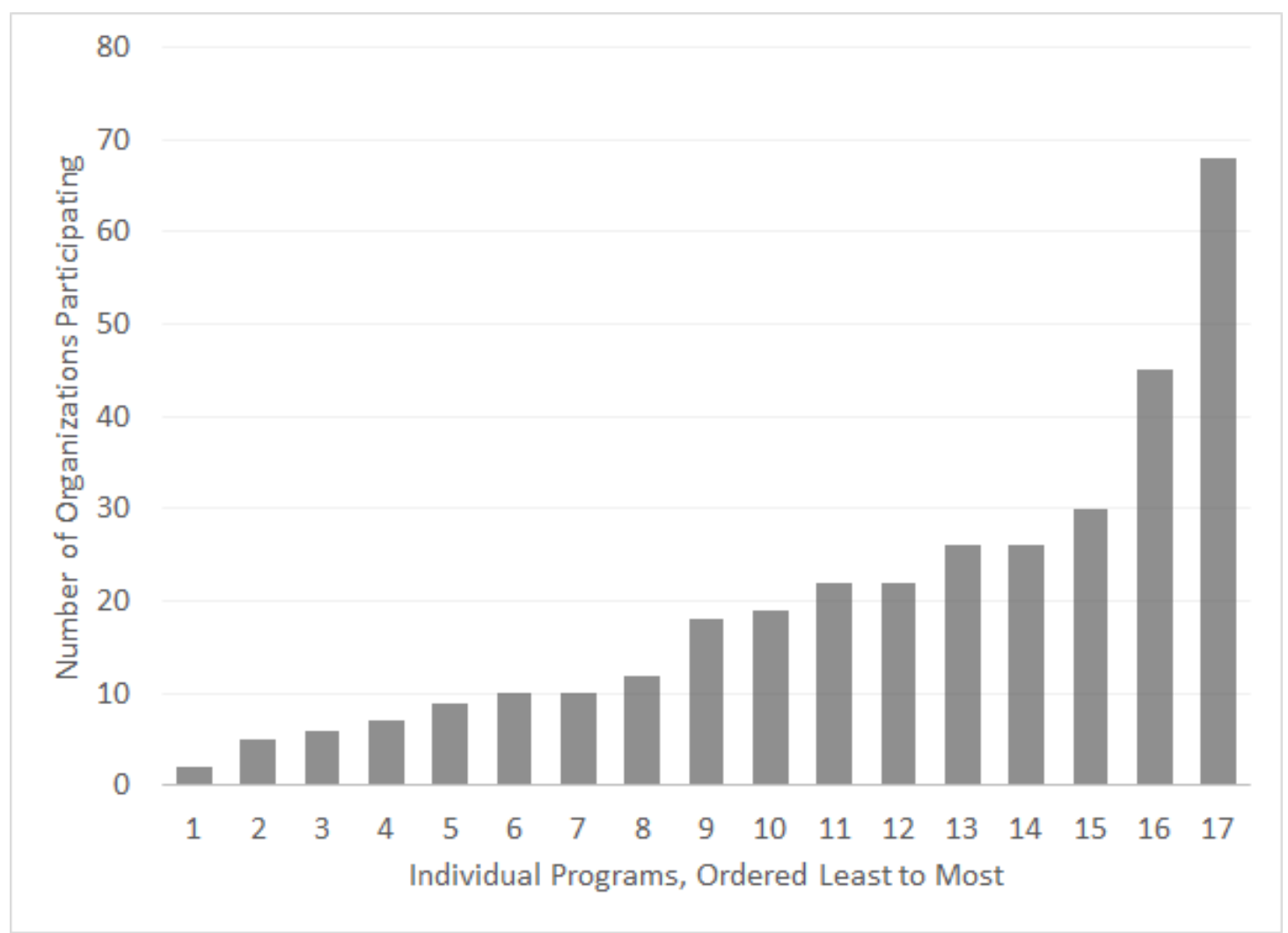

Figure 4. Number of organizations within each program.

Figure 4 provides a realative review of the size of each program based on the number of organizations represented within the program. The data was ordered from least to most. These organizations were identified within each of the 17 DHS Centers of Excellence program websites and fact sheets. The collective members of these organizations particpating in a program was the population for this research.

The number of years a program has been active were also of interest. The number of years a program was active was found through researching DHS announcements and reviewing program materials. Sometimes the information was stated in the program materials and other times it was derived from DHS announcements and publication dates. According to DHS material, their Centers of Excellence programs were expected to have 
no more than a 12-year lifecycle with annual assessments and renegotiations at a 3-year intervals. When a program no longer produced publications, it was assumed to have become inactive. Publications and supplemental programmatic information were used to determine the activity of programs. The two new programs announced in 2017 were not included in my study. Figure 5 provides a summary of the number of years for each program binned by the number of years. The programs ranged from a program being active for a single year to two programs operating for 13 years, with a mean of 6 years, median of 9 years, and mode of 3 years. From the data, it was apparent that most programs last approximately 3 years. The DHS Office of University programs generally added and removed programs collectively resulting in multiple programs starting and stopping at similar times.

It was important to understand the relationship between the years a program was active and the number of organizations engaged in the program. A nonparametric correlation evaluation between the affiliated organization information as it related to the number of active years for a program indicated a positive association, $(r(15)=.47, p=.22)$.

Lastly, I analyzed the programs for the number of publications listed. Figure 6 represents the number of publications for each program (see U.S. DHS, 2017). Several of the programs did not produce an identifiable publication on their website or in their materials. One possible reason for the absence of publications could be that some of the programs were targeted at producing technology rather than basic research and publications. Another reason may have been the short timeframe the program was active. 
There was also the potential the program did not produce publishable material because of security or classification restrictions. Understanding the number of publications produced by a program could be useful for understanding the drivers for the research in an academic environment, scientific prestige, and recognition. In many cases, these programs use graduate and postdoctoral research assistants' support for their research. In those cases, the students may be motivated by the process of conducting doctoral research or publishing to strengthen their identification within the academic community.

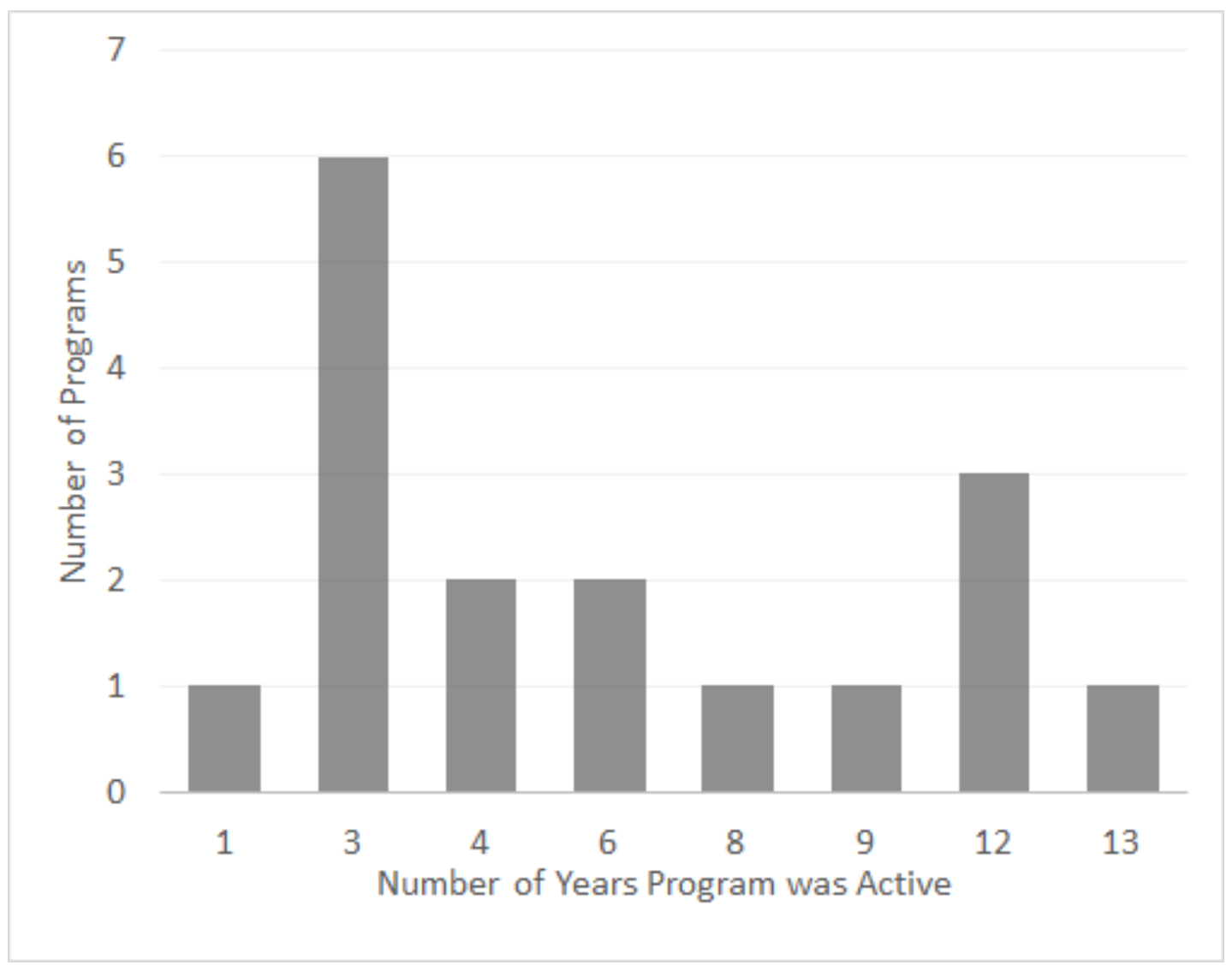

Figure 5. Number of years the program was active.

Figure 5 represents a chart summarizing the number of years each of the 17 DHS Centers of Excellence programs was active. The programs ranged from a program being 
active for a single year, upto a program operating beyond the original 12-year projections. Three years was the most common duration for the 17 programs, which aligns with the first program rebid cycle.

As Figure 6 shows, there is wide variability in the number of publications across the 17 programs, with a mean of 63 , median of 10 , and mode of 0 . This variability suggests there does not appear to be a DHS Centers of Excellence program-wide emphasis on publications. If the number of publications was an indicator of a program's research culture, then data suggest the culture of these programs might be varied, with many programs not emphasizing publications.

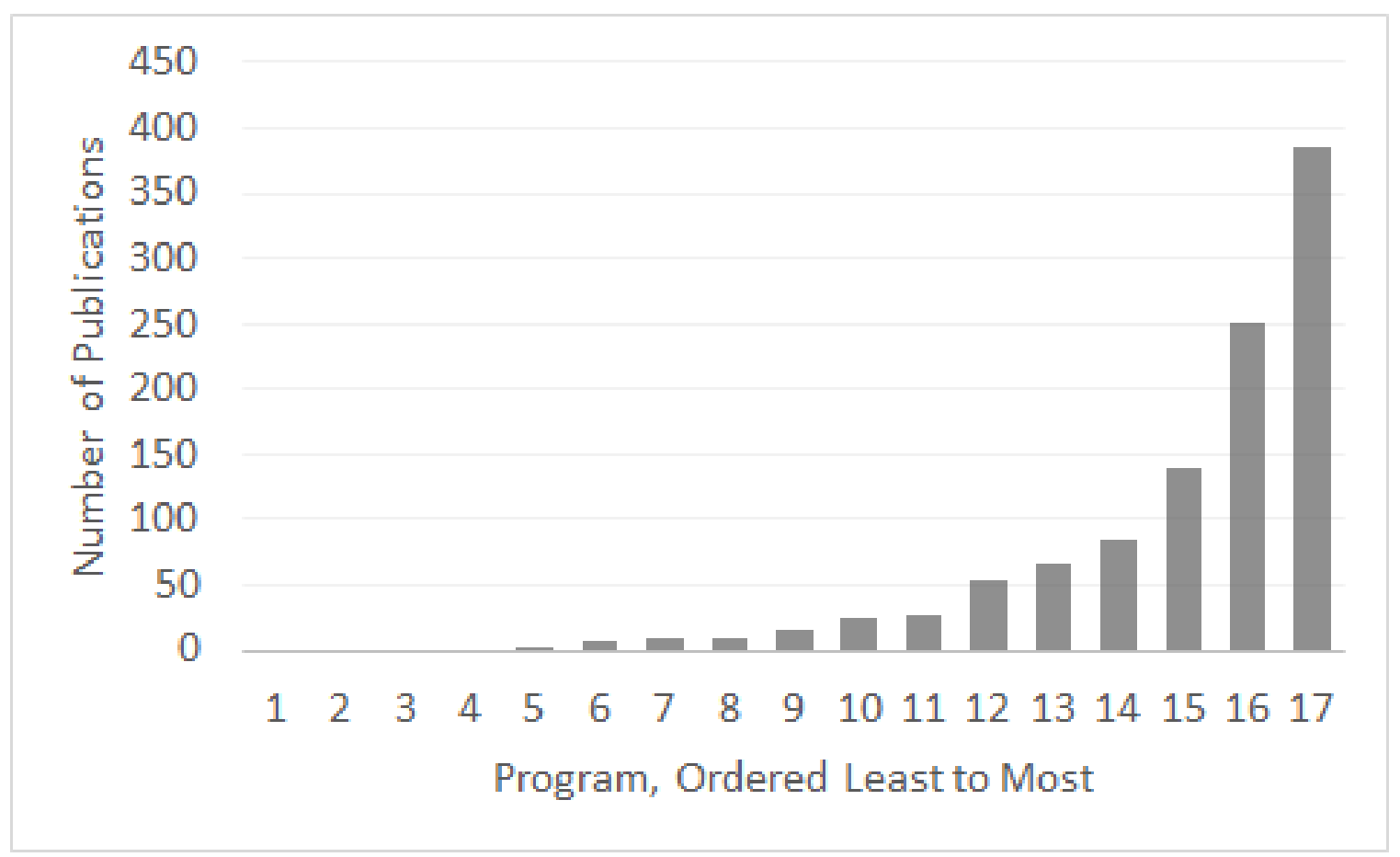

Figure 6. Number of publications listed within each program.

Figure 6 provides a summary of the number of publications each of the DHS Centers of Excellence program produced, according to their project website. The 
programs ranged from several programs listing no publications, to one program listing almost 400. The majority of programs listed less than 50 publications.

To further understand if program duration influences the number of publications, I explore the relationship between duration and publication counts. A nonparametric correlation evaluation between the number of years a program was active versus the number of publications it produced indicated a positive association, $(r(15)=.47, p=.22)$. This relationship may simply indicate that more publication was possible over more time. It may also indicate that publications are an indicator of program health. The role of publications in this research was an area of interest when analyzing the data gathered from participants.

Likewise, a similar exploration of the number of publications related to some organizations within a program was conducted. A nonparametric correlation evaluation between the number of publications with the number of organizations within a program indicated a positive but nonsignificant association, $(r(15)=.32, p=.1)$. There was spectulation that a higher number of organizations within a program might be associated with a larger number of publications.

The funding amount could be another potential indicator of program size, consistent information on specific funding allocations was not available for all DHS Centers of Excellence programs considered for my study. The final element analyzed to understand the research setting was the number of participants identified for each DHS Centers of Excellence program. The population for my study was the participants across 17 DHS Centers of Excellence programs. Figure 7 provides the information on the 
number of participants in each program ordered from least to most, with a mean of 65 , median of 88 , and mode of 33 . There were a varied number of participants ranging from 10 to over 200, with two of the programs being much larger than the majority of the other programs.

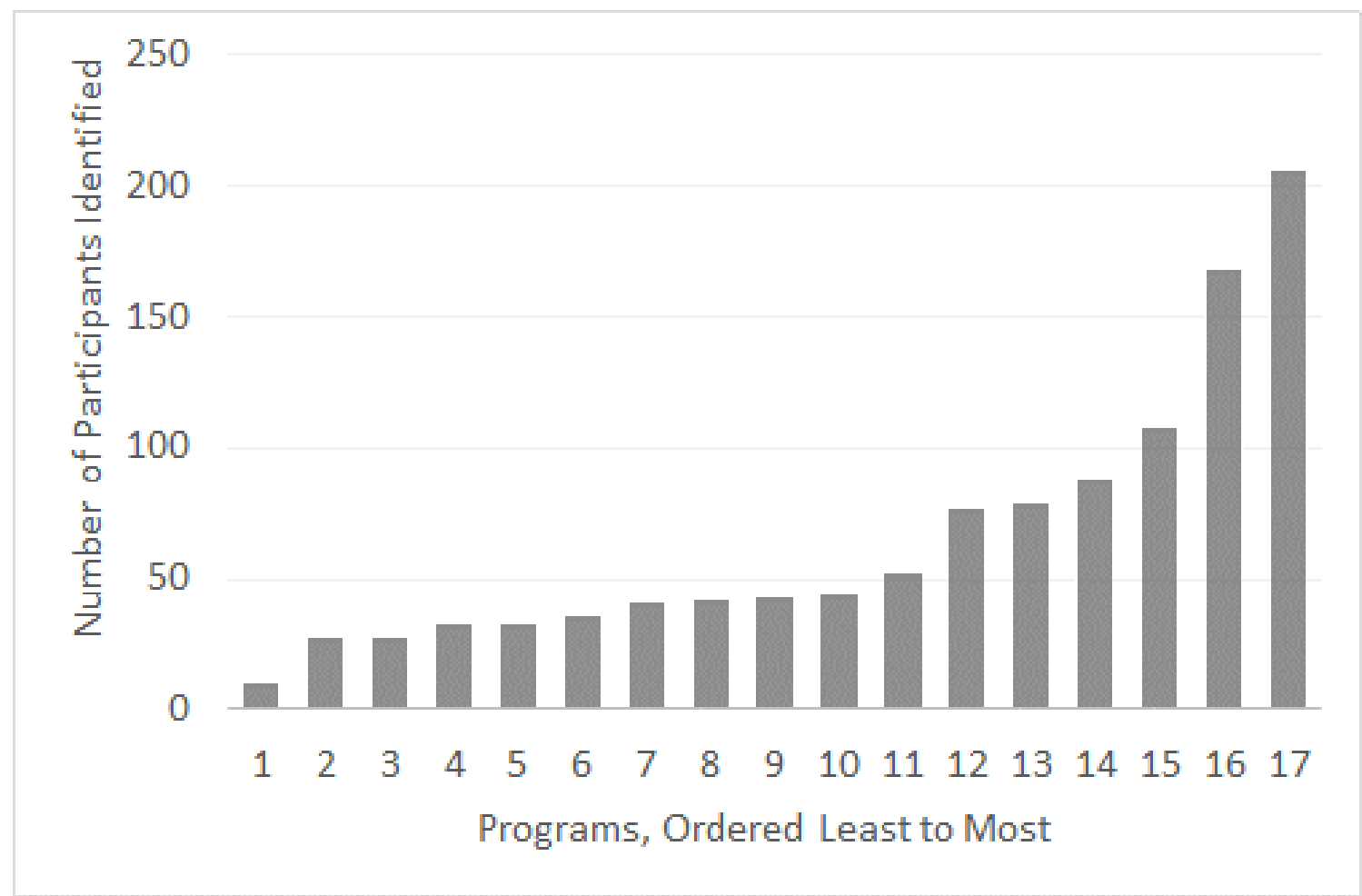

Figure 7. Number of participants identified for each program.

This figure provides a summary of the number of participants identified within each of the 17 Centers of Excellence programs ordered from least to most. This chart showed the number of participants identified under each of the DHS Centers of Excellence programs website and materials (DHS, 2017). The majority of programs included less than 100 participants. As expected, there was a positive but nonsignificant 
association between the number of participants and the number of organizations that were identified with the program, $(r(15)=.69, p=.48)$.

\section{Demographics}

The unit of analysis for my study was a program. An individual program was made up of multiple organizations collaborating as a single overarching organization. The unit of observation was the individual participant from an organization within the program. To fully characterize the unit of analysis, both participant and program demographic information was collected and considered for this research. Most of the participants in these scientific collaboration programs under the auspices of the DHS Centers of Excellence programs were university professors, administrator, graduate students, or postdoctoral research assistants. The demographics of the participant pool reflect the academic nature of the population. Figure 8 provides a hierarchical representation of the overall DHS Office of University Program's Centers of Excellence program structure at a high level. The participant pool included university leaders, research area leaders, project leaders, and research assistants engaged in the research. University leaders were individuals with national, and often, international recognition as experts in their field. Program participants are assumed to have completed 4 years of education and may begin supporting a DHS Centers of Excellence program as an administrator, faculty, graduate, postdoctoral research assistants or industry contractor. The participant pool included past and current participants in programs. Seven of the programs were currently active, four were moved to emeritus, and six are listed as former centers. Participants from all 17 programs were included in my study participant pool to 
allow for insights from continuing programs and past programs. In addition to participants listed on current program materials, participants were included in this study if they appeared on program publications to allow past participants to contribute.

$\begin{gathered}\text { Department of Homeland Security Science and Technology Directorate's } \\ \text { Office of University Programs }\end{gathered}$
Program Management
Center of Excellence
University Lead
Project Research Area
$\begin{gathered}\text { University Partner } \\ \text { Researchers }\end{gathered}$

Figure 8. Hierarchy of the DHS Centers of Excellence programs.

This qualitative case study included 15 program member interviews. The participants interviewed represented 10 DHS Centers of Excellence programs. The participants represented 12 organizations supporting those programs. All of the participants conducted research as part of multiple organization during their research experience. For my demographics, I included the participant's organization identity during the time they worked with the DHS Centers of Excellence program. The participants interviewed included representation from the university lead, faculty, industry partners, graduate students, and postdoctoral research assistants. Two of the participants served as assigned leadership within a program, two industry partners researching within a program, and 11 participants were either faculty researchers or 
students during their research time within the program. Two of the participants interviewed had participated as both a student researcher and a faculty under their program.

The participants interviewed represented the highly educated nature of the programs. Thirteen of the 15 participants interviewed had achieved a Ph.D. Three participants had started their research within the program before receiving this higher degree. Their research topics represented by the participants were vast and varied. All of the participants represented more than one discipline area. They represented a variety of expertise such as chemistry, computer engineering, policy and many others. Figure 9 provides a histogram of the years of research experience represented by the participants in my study. Insights were gathered from participants with a range of years of research experience from as little as 4 years to almost 40 .

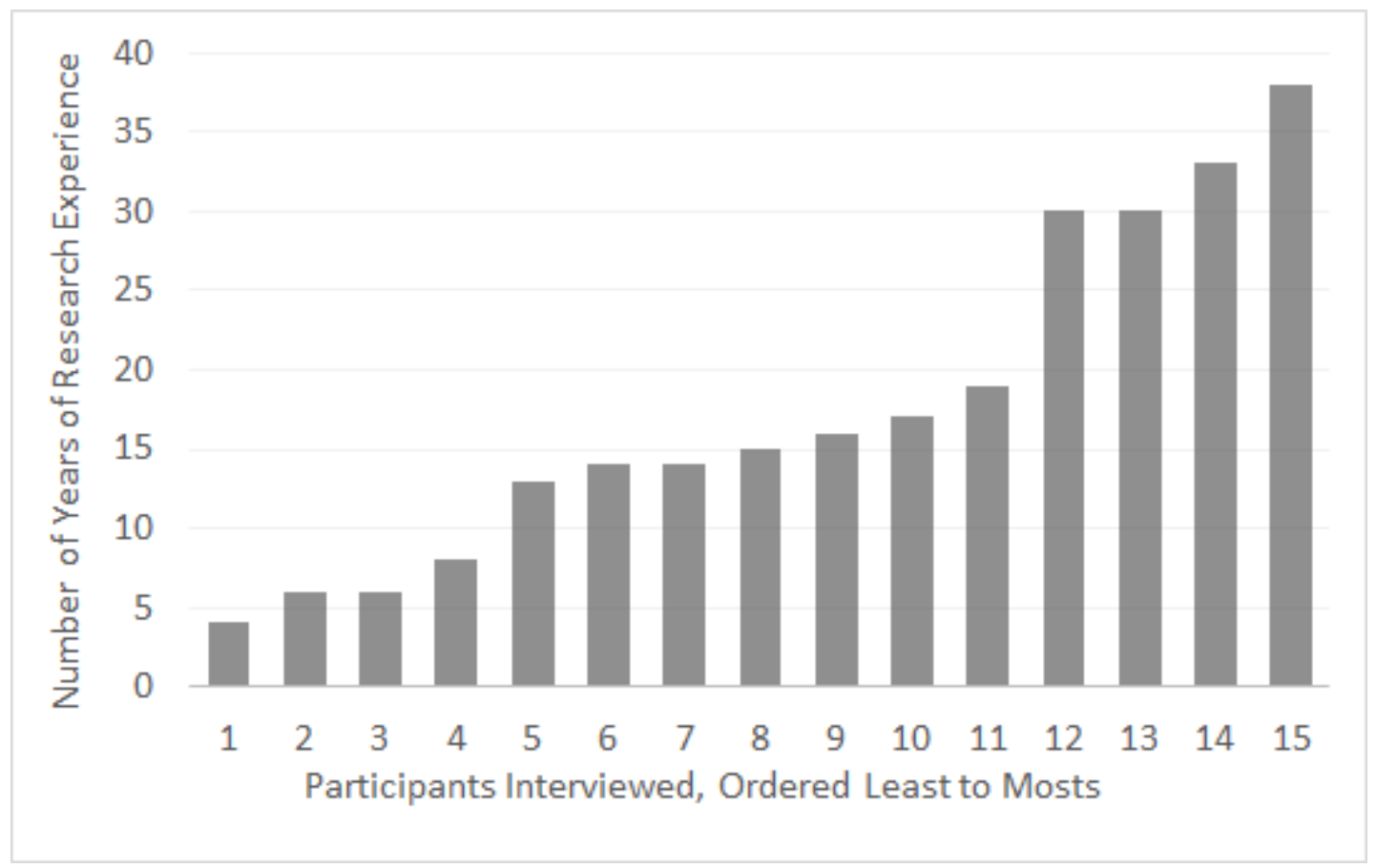

Figure 9. Years of experience of each participant interviewed. 
These programs operate in an academic environment. The participant pool availability could be influenced by the academic calendar year. Participants may have had less available time during the summer months when school was not in session. Other may have had more availability during the summer months when class/teaching loads were light. To maximize participation this research study, I conducted participant recruitment over both the summer and school-year timeframes.

\section{Data Collection}

An exploratory semistructured interview was conducted with participants sampled from across the DHS Security's Centers of Excellence programs. This qualitative research study consisted of individuals who volunteered to participate in interviews conducted over the phone. This data collection format allowed for flexibility in scheduling interviews convenient for the participants. Interviews were within one hour unless the participant requested an extension. The full set of DHS Centers of Excellence programs was explored to provide insight into leadership in multiorganizational scientific collaboration programs. This study followed a qualitative case study research design. A set of semistructured interview questions was developed following guidelines put forth by Seidman (2013). Figure 10 provides a full workflow of the overall data collection and analysis preparation process. The workflow included identifying, gathering, and contacting potential participants for this study. 


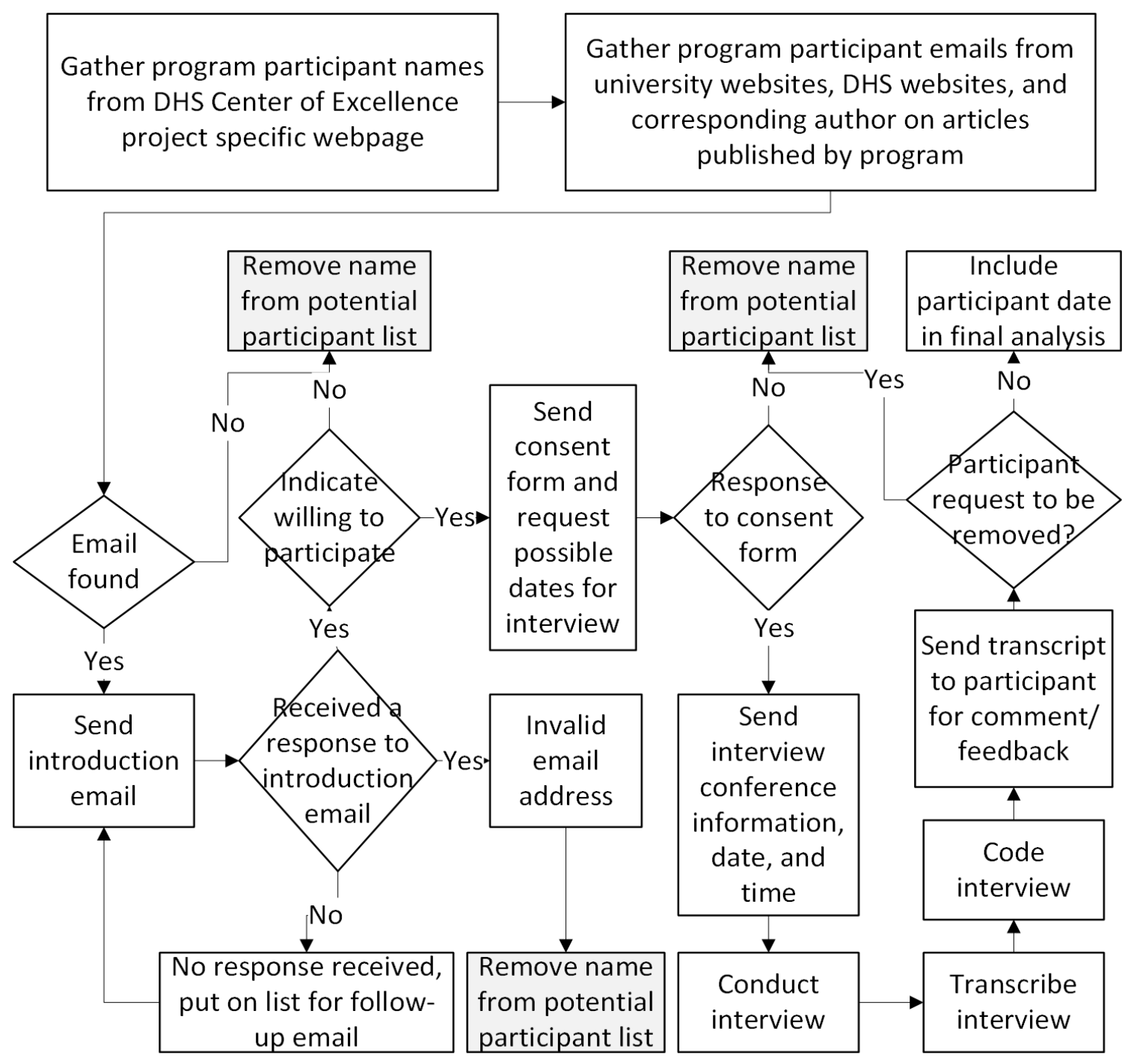

Figure 10. Data collection and analysis preparation process.

Figure 10 includes the participant identification, communication, selection, interviews, and post-interview processes. Reading from left to right a program members' participation was determined after they have been identified and respond to the e-mail inquiries. From there, partcipants receive a consent form and the interview questions to preview. After each interview, the information was transcribed, coded, and returned to the participants for member checking. 


\section{Sampling Selection}

This qualitative case study was intended to represent by the collection U.S. DHS's Centers of Excellence programs. The unit of analysis for my study was a program. An individual program was made up of multiple organizations collaborating as a single overarching organization. The unit of observation was the individual participant from an organization within the program. A convenience sampling approach was used to recruit individuals to participate in the research.

The sampling process began with an investigation of information available online regarding a particular program. From there a list of potential participants was derived from program information on its members. Program publications were also used to find individual contributors to program products. After having collected names, a search for emails was conducted. First searching the program site itself, and then searching university sites where individuals were identified as being part of a university. Finally, a search was conducted of publication websites where the participant was listed as the first author. In many cases, the first author had the potential to provide an author correspondence e-mail. After conducting these searches, if no e-mail was found, then the potential participant was listed as having no e-mail available. At that point in time, any potential participant with an e-mail located was sent the request for participation. Figure 11 provides a breakdown of the number of potential participants identified within the 17 DHS Centers of Excellence programs included in this research case study. More than 1,000 participants were identified across all programs; with e-mails being sent to those with identified e- 
mails. Still, some e-mails were not deliverable and were removed at the request of the program participant. A second request was sent to individuals who had yet to respond.

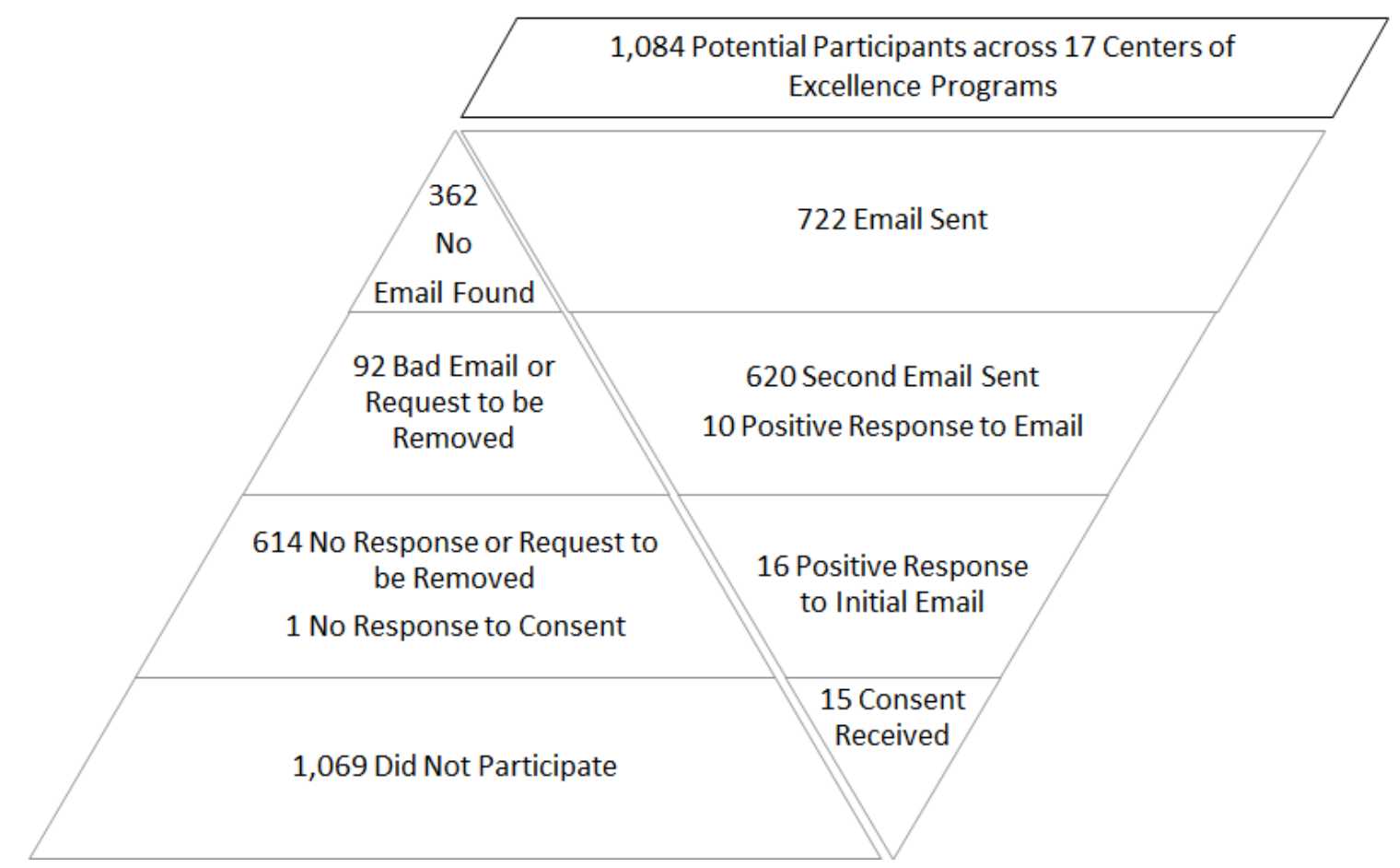

Figure 11. Summary of participation and responses tracking.

There were three types of responses from the request to participate. First, an undeliverable e-mail was received. In this case, the e-mail was found to be invalid or no longer in service, and the potential participant was removed from future mailings.

Second, a returned e-mail from the potential participant asking to be removed from consideration for my study was received. When a request for removal was received, I removed the name from the potential participant list. The third type of response was a returned e-mail from the potential participant indicating a willingness to participate in my research. In this scenario, a second e-mail was sent to the participant containing the consent form, interview questions, and a request for options of times for the interview. In 
many cases, these consent e-mails were not returned. After a follow-up e-mail was sent, if a consent return e-mail was not received, the individual was removed from the study participant list. A second e-mail requesting participation was sent to boost the response rate. Any subsequent response received was treated in the same manner as the original response.

This qualitative case study included 15 program member interviews representing different 10 multiorganizational research and development programs under the DHS. This sample included individuals with a variety of roles to obtain maximum heterogeneity. Through exploratory semistructured interviews, this study could guide the development of insights into leadership constructs of programs, such as the DHS Centers of Excellence programs. Without significant preexisting research in this area to guide a survey of followers, a case study approach allowed for open-ended interviews as the main method of gathering information about followers' perspectives on leadership. Additional documentation and additional individual opportunistic interviews were allowed as time permitted.

Data collection included interviews and collection of organizational documentation. All references to names of organizations and people were coded in the transcript to avoid connecting specific statements to individuals and organizations. In the case where a single individual was identified in more than one program, they received only one invitation to participate. 


\section{Data Gathering}

Interviews provided an opportunity to understand the perspective of the participant. This approach gave the participants a chance to share experiences, stories, observations, and ideas based on their experience in the area of scientific collaboration. Responses to open-ended questions were parsed into specific response statements, which become the raw data for the analysis. Because the interviews were conducted over the phone where body language and the environment could not be observed, it was important to minimize the amount of discussion time the interviewer was speaking. One method for helping to maintain the integrity of the information gathered was to allow the participant to elaborate with as little guidance and probing by the interviewer as possible. I used targeted probing questions to gather thicker discussion and draw out deep thought.

Interviewing as part of this qualitative study was effective in understanding if there were naturally emerge commonalities across program participants' information. Elements such as program structure, its implementation in a virtual environment, and its impact on participants were a few areas investigated during this data collection effort. My study used semistructured open-ended interview questions. These types of interviews involved individuals. They were one-time sessions lasting up to an hour, and the interviewees did not participate in additional interviews. During the interview, the semistructured interview guide was used (see Appendix A). Each interview session was recorded so that I, as the researcher, could actively capture ideas and pose additional probing questions during the interview session. Both handwritten notes and audio 
recordings were used so to focus on the interview content and ensure I had captured each interview in its entirety.

All interview recordings were transcribed and coded. Transcripts along with coding associated with the interviews were provided to the research participants for their elaboration, correction, or removal from the study. This member checking allowed the participants to review the information captured during the interview session and ensure that I, as the researcher, had accurately captured the participants' experiences.

\section{Data Saturation}

Data saturation was a current topic among qualitative researchers (Hennink et al., 2017; Malterud et al., 2016). Saturation was achieved when no new themes, perspectives or insights were evident when additional data was gathered and reviewed (Patton, 2015). According to Fusch and Ness (2015, p. 1408), "data saturation was reached when there was enough information to replicate the study, when the ability to obtain additional new information was attained, and when further coding was no longer feasible." Qualitative data gathering and analysis were subjective with multiple possible interpretations. Much like qualitative study itself, the researcher played a large role in defining and defending the evidence for saturation. Factors playing into the determination of saturation were the purpose of the study, research design, characteristics of the population being studied, analytic approach to analysis, and available resources (Hennick et al., 2017). The purpose of my qualitative investigation was to gain an understanding of followers' perspectives regarding virtual leadership within complex multiorganizational programs. My research design was a qualitative case study. My population of interest was participants in 
multiorganizational scientific collaboration programs made up of highly educated individuals. My analytic approach used a case study design with an iterative analysis methodology to guide findings and identify saturation.

Several researchers have indicated the need for thick dialog when conducting interviews (Fusch \& Ness, 2015; Malterud et al., 2016; Patton, 2015). Malterud, et al. (2016) referred to this as the quality of the dialog and scored it as either weak or strong. Fusch and Ness (2015) discussed interviews in terms of rich to represent quality and thick to represent quantity. My research interviews were predominantly strong in-depth dialog. According to Malterud et al., a strong dialog would require fewer interview sessions than a weak dialog.

The quality of the dialogue could also be impacted by the depth of understanding the researcher has regarding the subject being discussed (Fusch \& Ness, 2015; Malterud et al., 2016; Stake, 1995; Yin, 2014). Stake's (2006) expectation on the appropriate use of case study research was dependent on the researcher's expertise in interviewing, analyzing, and revisiting research processes throughout the case study. In my research, my extensive experience as a researcher in an academic-like environment enables me to understand the general purpose and operating environment of most of the potential participants. Demographic and background questions were asked at the beginning of each interview to provide a basis for understanding each participant's perspective and operating environment.

Understanding the point of completion and the study goals well enough to remain on track during this iterative saturation process were viewed by Stake (1995) as daunting 
and may not be possible for researchers new to the method. Yin's (2014) structured methodology provided a basis for planning and conducting case study research. One of Yin's strengths was providing the researcher a variety of methods for collecting and organizing data to aid in the systematic review and reporting of findings. Within my research, I am very familiar with collaborative scientific environments, working with highly educated individuals in an academic environment, and working virtually with colleagues. In order to limit personal bias, journaling, limited researcher speaking, and member checking were used to moderate personal ideas were identified and managed during analysis.

\section{Data Analysis}

Data analysis included program information, interviews, and researcher notes. Information was taken from the program online resources regarding the purpose, size, and duration of the program.

\section{Code List}

Code lists were initially developed from extant literature and DHS (2015) program expectations. The literature review consisted of the current literature search, review, and synthesis. The literature review extended to complex system leadership (Henry, 2015; Matzler et al., 2016), scientific collaboration (Leiserson \& McVinney, 2015), and leader-member exchange theory (Schermuly et al., 2015; Erdogan \& Bauer, 2014; Long, et al., 2013). Program documentation was gathered to provide background on program mission, size, and organizational structure. Potential codes originally 
included concepts around fostering creativity, crossing organizational boundaries, encouraging scientific collaboration, and providing customer-driven solutions.

In addition to code list development, the exploration of previous literature related to multiorganizational research and development program leadership guided common terminology potentially used by participants. For example, Northouse's (2016) description of identified traits as a starting point for coding interview transcripts. This terminology also served as the basis for the development of the initial semistructured interview questions used. Appendix B provides the initial code lists and final code lists for my study.

Two-cycle coding was used to identify elements of each interview, and then to look for patterns across interviews (Miles et al., 2014). The initial code list was prepared from the literature review process and represented terminology from previous research. During the first cycle of coding, codes were added or removed from the code list depending on their applicability to the interview content. In the first cycle coding, codes were originally noted while listening to the interview recording before transcription. Codes were also added to notes taken by me during the interview. A summary memo was written immediately following the interview to try to capture notes and initial thoughts.

After the interview was transcribed, coding was used to categorize the transcript text. Each portion of the text was individually coded and captured as comments in the document. This annotated transcription was provided to the interviewee for comment and elaboration. Each portion of the text was also placed in a spreadsheet and tagged with the 
appropriate code(s). This was intended to allow analysis of interviews by code across interview content.

During second-cycle coding, the code lists emerging from the notes and transcripts were reviewed for patterns and relationships. The spreadsheet developed at the end of the first coding cycle was used to explore the content of all interviews. Code filters were used to review transcript content for themes and patterns. Codes were reviewed individually and in groups of potentially related codes. For example, one code on prestige might be interesting to explore when cross-referenced with publications/publishing to see if a possible theme emerged.

Miles et al. (2014) suggested three ways to communicate coding in research using narrative descriptions, matrix display, and network displays. For my research, I used all three methods to some degree to explore and communicate observations. These methods were used to help group and review interview content during the second cycle pattern analysis. Network displays of patterns were used for specific relationships that were identified. A prosaic presentation of findings was provided here as a narrative description of findings associated with interview content.

As data collection and analysis continued, additional concepts emerged, such as the role of the post-doctoral researchers in collaboration. My process for building, modifying, and reporting codes was based on approached recommended by Hennink et at. (2017) to track code emergence over time during collection and analysis. Tracking the codes provided some transparency for analysis as well as a way to visualize possible saturation. Therefore interviews were tracked as A, B, C, etc. to indicate the order in 
which they were conducted. A matrix display of the codes and their related interview relevance was used to explore the content of the interviews across all participants.

The number of participants in my study guided the type of analysis conducted. Having a limited number of interviews eliminates the option of using semantic data analysis tools on the interview transcript data. Semantic tools could be used to identify common themes and discover relationships in information in unstructured text formats, however, these tools were statisticly-based and generally required a significant number of documents to provide a reliable output (Potel \& Wong, 2014).

I analyzed the themes emerging throughout the interview process using a collective spreadsheet to represent all codes and associated participant statements across all interviews. This resulting visual analysis provided me the opportunity to identify potential saturation, note emerging themes, highlight outliers, and clearly identify which codes from the pre-interview code list sustained.

\section{Researcher as Instrument}

The instrumentation for my study consisted of a set of semistructured interview questions with me as the researcher severing as the instrument of analysis. This study focuses on the followers' perspective regarding virtual leadership. Researchers have previously looked at the transformational leadership component of leader processes (Cole et al., 2011). Leadership efficacy from the leader's self-assessment perspective was also previously studied (Hannah et al., 2012). Previous approaches focused on leader selfassessment rather than the followers' perspectives of the leader. Erdogan and Bauer (2014) suggested future researchers step away from the commonly used seven-question 
instrument. These researchers recommended using exploratory methods when seeking to understand members' relationships with the leader. No available instrument was appropriate for follower perspectives of leadership in virtual scientific collaboration environments. I did not find in the extant literature an instrument to assess complex multiorganizational research environments.

The interview transcript reviews were conducted in the order in which they were collected to assess codes and possible saturation. For each interview, new codes were identified and recorded. I also documented code characteristics such as code name, code definition, code type (inductive or deductive), any notes about new codes (e.g., clarity of the issue, completeness of the code definition), which existing codes were present in the interview. Each code definition included a description of the issue it captured, criteria for code application and any exceptions, and an example of the text relevant to the code. To identify the evolution of the code development, I recorded any changes made to codes developed in previous interviews, including the nature of the changes and interview number at which each change occurred.

This code development documentation and iterative refinement of codes continued for each interview until all interviews were reviewed and the codebook was complete. Codes were then categorized for analysis as follows. First, codes were categorized as inductive or deductive. Deductive codes were researcher-driven and originated from the interview guide. Inductive codes derived from the interview itself were denoted with bold text. Codes that were not used had a strikethrough notation added. Changes to the codes were categorized as a change in a code name, change in 
code definition, code merged, and code split into separate codes. Code definitions changes were further categorized as expanded conceptually, added examples, edited, inclusion/exclusion criteria, and added negative component. Lastly, codes were also categorized as process and descriptive. Process codes captured an action or relationship among descriptive code concepts as shown in Figure 12. Each participant's key codes are provided in a columnar view cross-walked with the process code list.

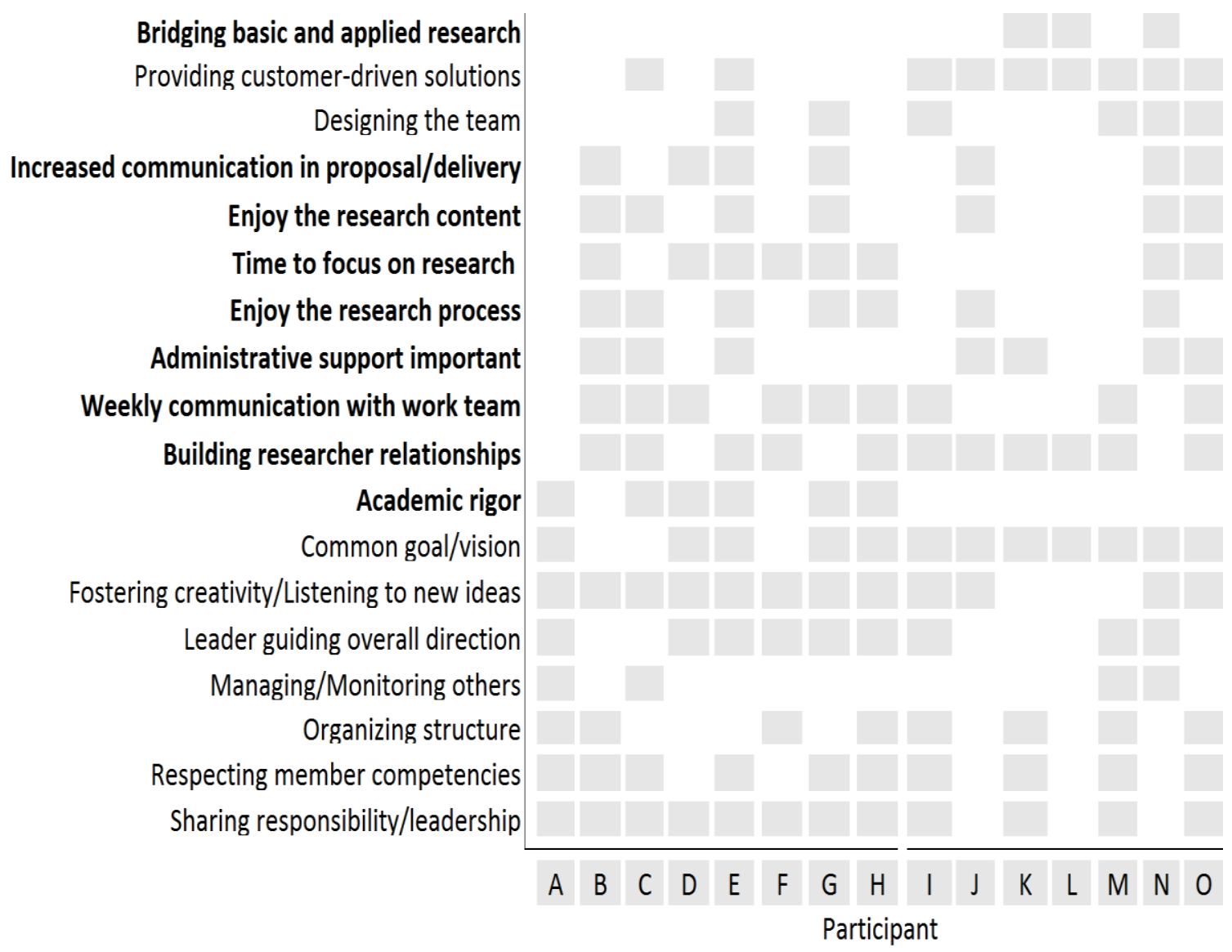

Figure 12. Process code listing by participant.

Descriptive codes were those capturing explicit, definitive issues in data; for example, the code "funding" captured concrete issues such as finding size. Deductive 
codes were developed prior to the data collection. Inductive codes emerged during the data analysis and are shown in bold typeface. This chart can be used to look for areas of agreement. For example, fostering creativity was a common theme, although not all participants emphasized it. The result of this process is depicted in Figure 13. Inductive codes are bolded to indicate their emergence during the data analysis. This chart can be used to look for areas of agreement. For example, publishing was a concept discussed by most participants as a role in the virtual leadership. Each participant's codes are provided in a columnar view cross-walked with the descriptive code list.

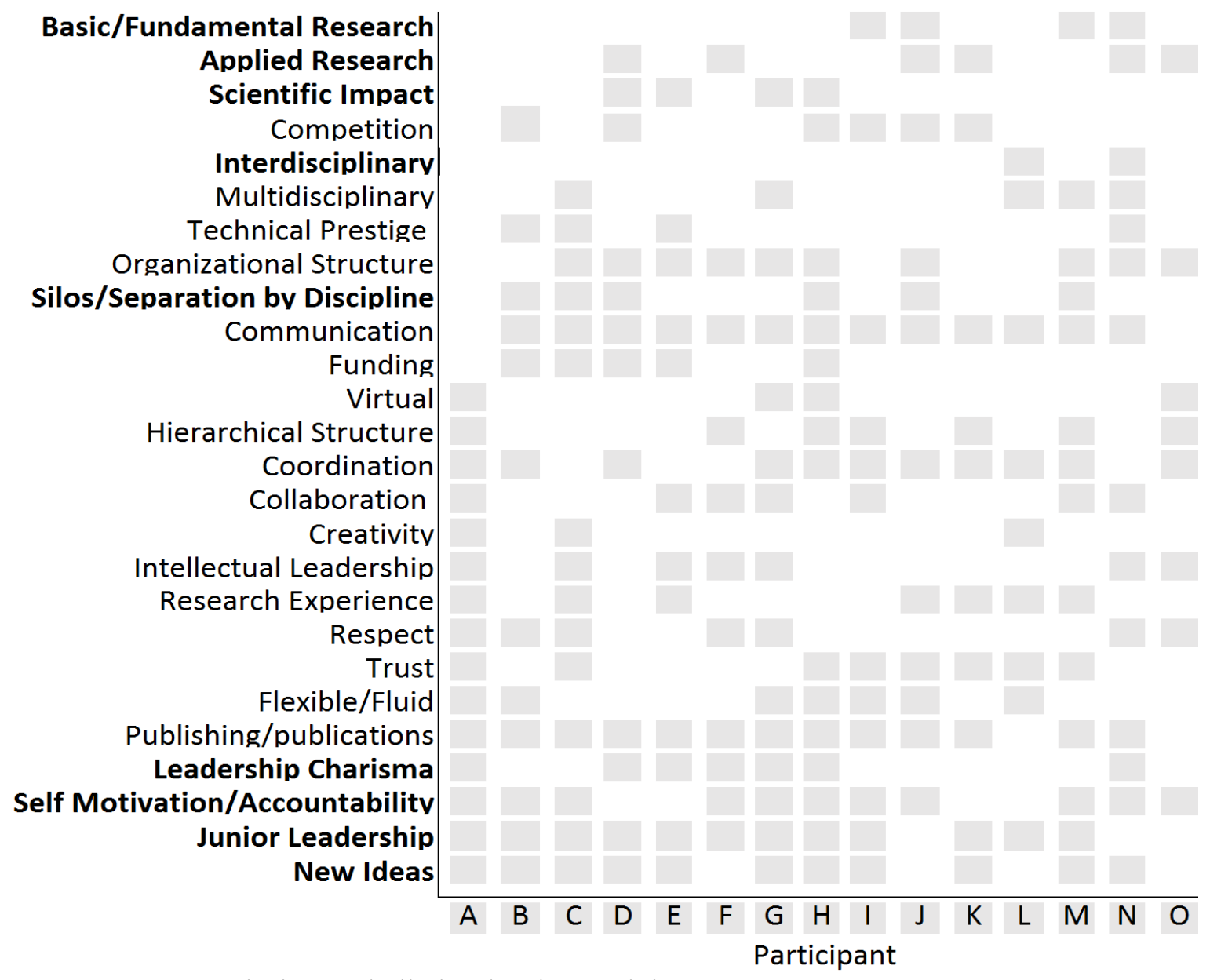

Figure 13. Descriptive code listing by the participant. 


\section{Evidence of Trustworthiness}

\section{Credibility}

For this research, credibility reflected quality and accuracy of information. I defined quality as demonstrating transparency in the process with an insightful evaluation of the information gathered. It was important to document each step in the process with comments to provide traceability. An important aspect of quality related to credibility involves the accuracy of interview transcription and interpretation. I transcribed the interviews and applied with initial coding. The coded interview transcription was returned to the participant to ensure the intent and observations were accurate. This member checking approach allowed the participants, if they were interested, to fully engage in the interpretation of their contribution before completing the coding process. In addition to ensuring data collection quality, process, and analysis methods were also documented to support the defensibility of my research processes and findings.

\section{Transferability}

Transferability for my researcher was an indication of the external validity of my findings. Miles et al. (2014) recommended clarity in information regarding the research as the best way to ensure optimal transferability. For this case study, detailed descriptions of the sample, setting, processes, and analysis techniques were provided in this report to increased potential for transferability. Transferability increased by providing thick descriptions. Finally, processes or results congruent with previous research were clearly noted. 


\section{Dependability}

Dependability was a measure of the quality of the research and the assurance the research was conducted appropriately. Several review questions, covering similar target topics, aided in addressing potential issues with quality and ethical concerns. Collection of participants representing more than one program was important to gain dependability and aid in the results being representative. Participants from 10 programs were used for this analysis when participants' representation was analyzed in relation to the participant pool for a check of completeness. Findings were critically reviewed for categories of interest and discrepancies. I used questions about the conditions under which the emerging theme(s) hold up to ensure the concepts relate to the findings.

\section{Confirmability}

Several activities for identifying and reporting bias were implemented to enhance confirmability of the research I used journaling to capture my initial and changing perspectives. I also collected member's input through interview questions. I used the process of transcription, coding, and pattern identification to verify logic and defensibility. Reviews were iterative to allow for updates to coding and revisiting any patterns identified.

\section{Study Results}

My research was guided by a single overarching research question: What virtual leadership strategies do leaders of interdisciplinary collaborations use to motivate their highly educated scientists across organizations? To further inquire about specific elements of my research question, I broke down the question into four subthemes: 
leadership, program structure, virtual environment, and research culture. Figure 14 visualizes the basis for the four subthemes chosen. The research findings and results discussion are organized by subthemes. The exploration of the data gathered from results of semistructured interviews with program members yielded four main findings.

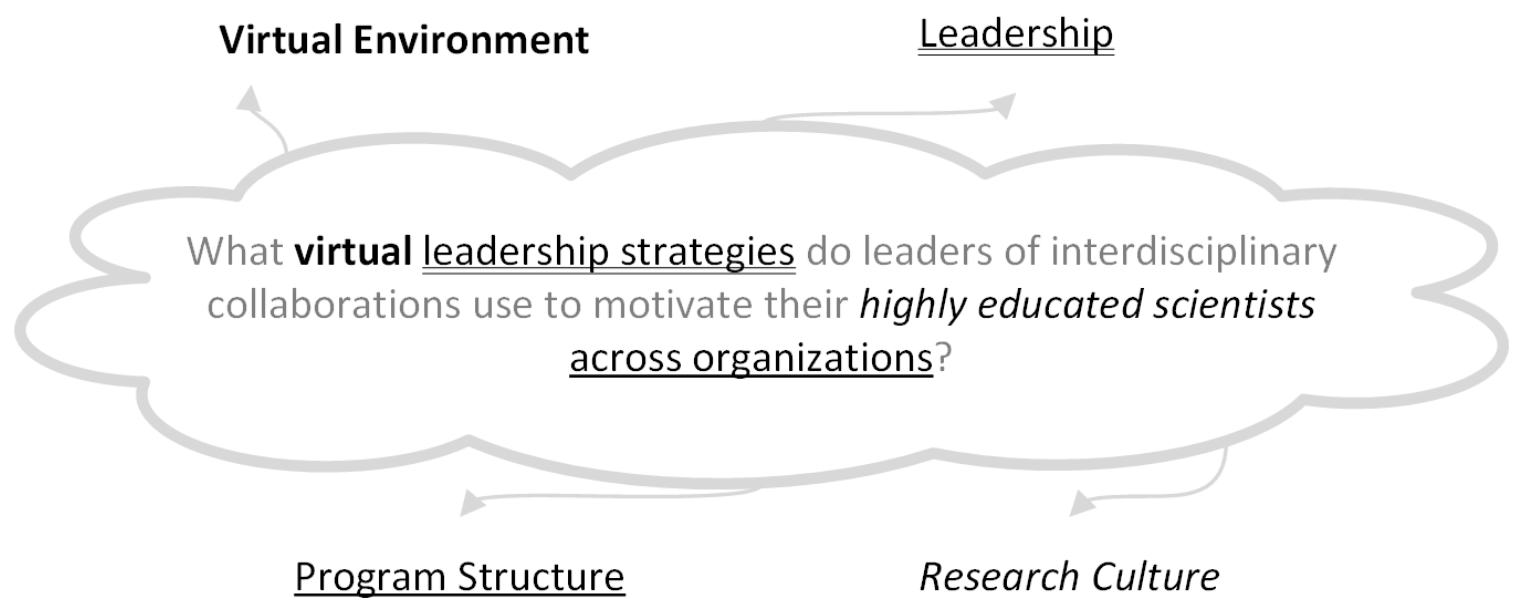

Figure 14. Four subthemes of research question.

The following results provide insights regarding the participants' view of leadership within multiorganizational research and development programs. I discussed learning related to program structures and the potential impacts of those structures. I propose an engagement framework providing a high-level view of programs and their drivers for collaboration. Lastly, I discussed teaming within the academic environment, noting the specific nonvirtual teaming observations found in my study. My study included 15 participants representing 10 DHS Centers of Excellence programs. These programs collectively represent more than 100 organizations. Added altogether these programs listed 145 organizations, although several universities were represented in more than one program. Figure 15 provides the number of organizations affiliated with 
the 10 programs represented by the 15 participants in my study. The number of organization within these 10 programs ranged from less than 10 organizations to almost 70 organizations. It was important for this study to ensure participants represented a variety of program sizes. These organizations were typically universities and other research organizations. The number of organizations was gathered from the Centers of Excellence program website and program fact sheets. In reviewing Figure 7 along with Figure 15, there does appear to be a reasonable representation of program sizes. The number of organizations for each program has been provided in order of least to greatest.

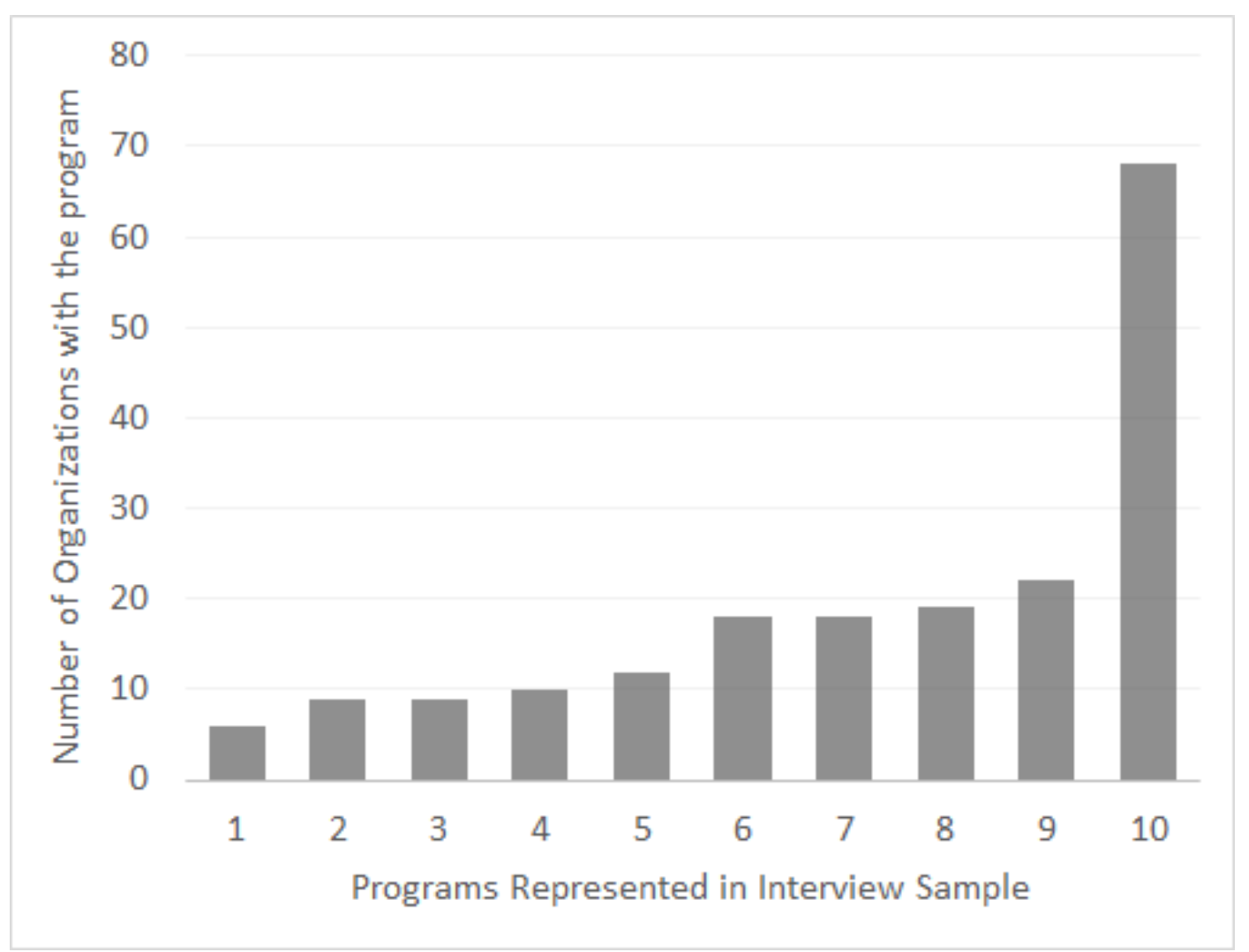

Figure 15. Number of organizations represented by the participants interviewed. 
Program longevity might be an indicator of successful leadership model. The number of years each program was active was collected from program materials provided by DHS (2017). Figure 16 provides the number of active years for each the 10 programs represented by the 15 individuals interviewed. The programs represented by my study range from 3 years to 13 years in duration. A distribution of short, mid, and longer-lived programs was represented.

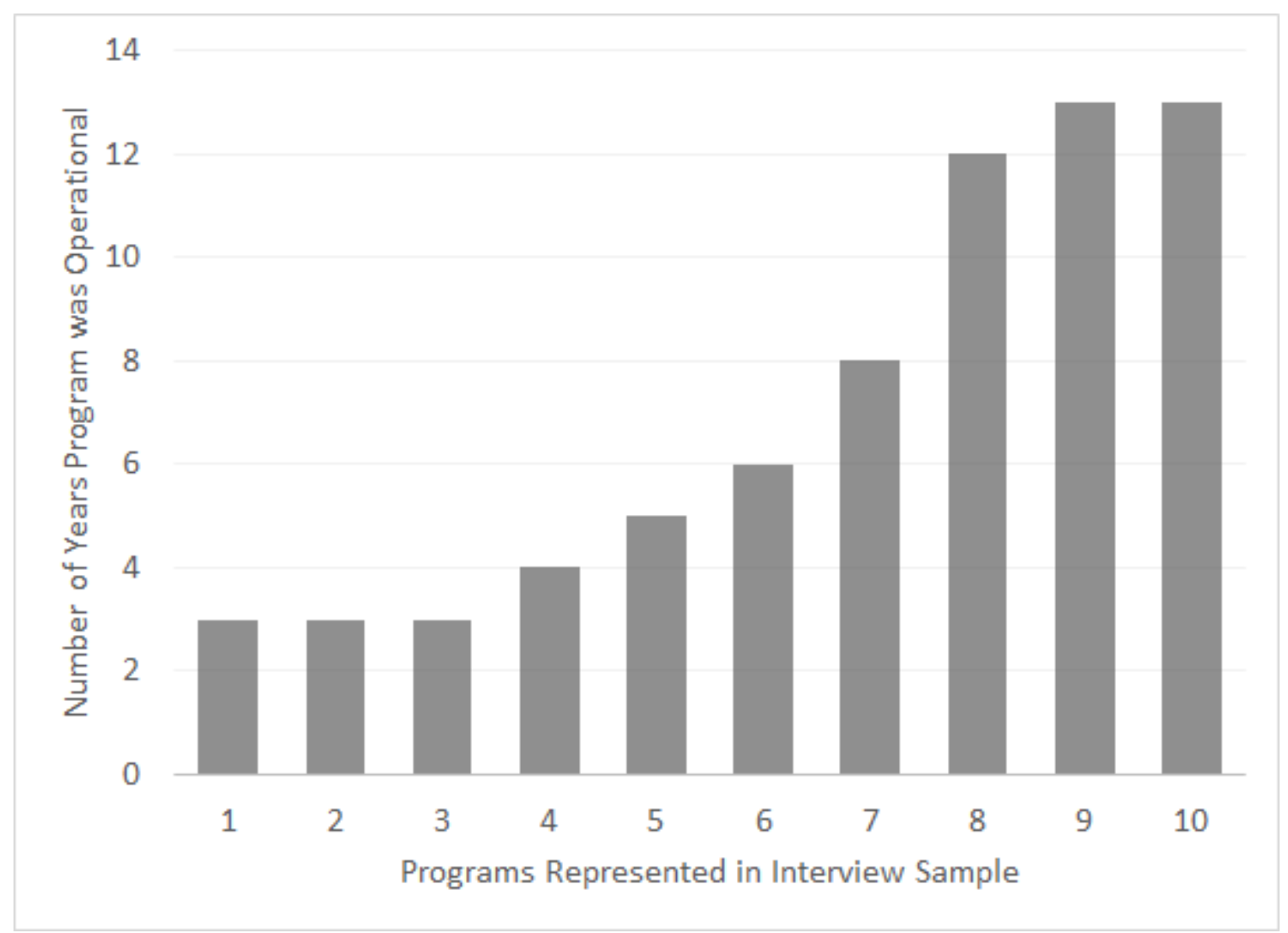

Figure 16. Number of operational years t represented by the participants interviewed.

Even with a limited participant set, the 15 participants opting to be interviewed appear to reasonably represent both small and large programs. Likewise, they come from both short and long-lasting programs. Finally, the interview participants represent 
programs with both few and many publications recorded. Number of operational years $\mathrm{t}$ represented by the participants interviewed

Fifteen individuals representing 10 programs chose to participate in my research. During the participant interview process, demographics questions were asked to understand the participant's role in the program. Information was collected for each participant regarding discipline, highest degree achieved, and the number of years the participant had been a researcher. The role(s) of each participant was discussed to understand if the participant had experiences as a program director, technical lead, or early career member.

\section{Subtheme 1: Leadership}

The exploration of the participant input yielded three aspects of leadership. The main tenants of the finding under this subtheme were transformational leadership (Qu et al., 2015), shared leadership (Hoch \& Kozlowski, 2014), and an emerging area noted as early career transactional leadership. Where transactional leadership was an element of shared leadership, most early career members of the team operated in a leadership role guiding research and conducting research elements. A feature of my research that may further distinguish scientific collaborations from other types of multiorganizational programs is the existence of early career leadership. Figure 17 provides a visual aid to understand the leadership theme, associated concepts and codes, and the basis for the finding provided here. 


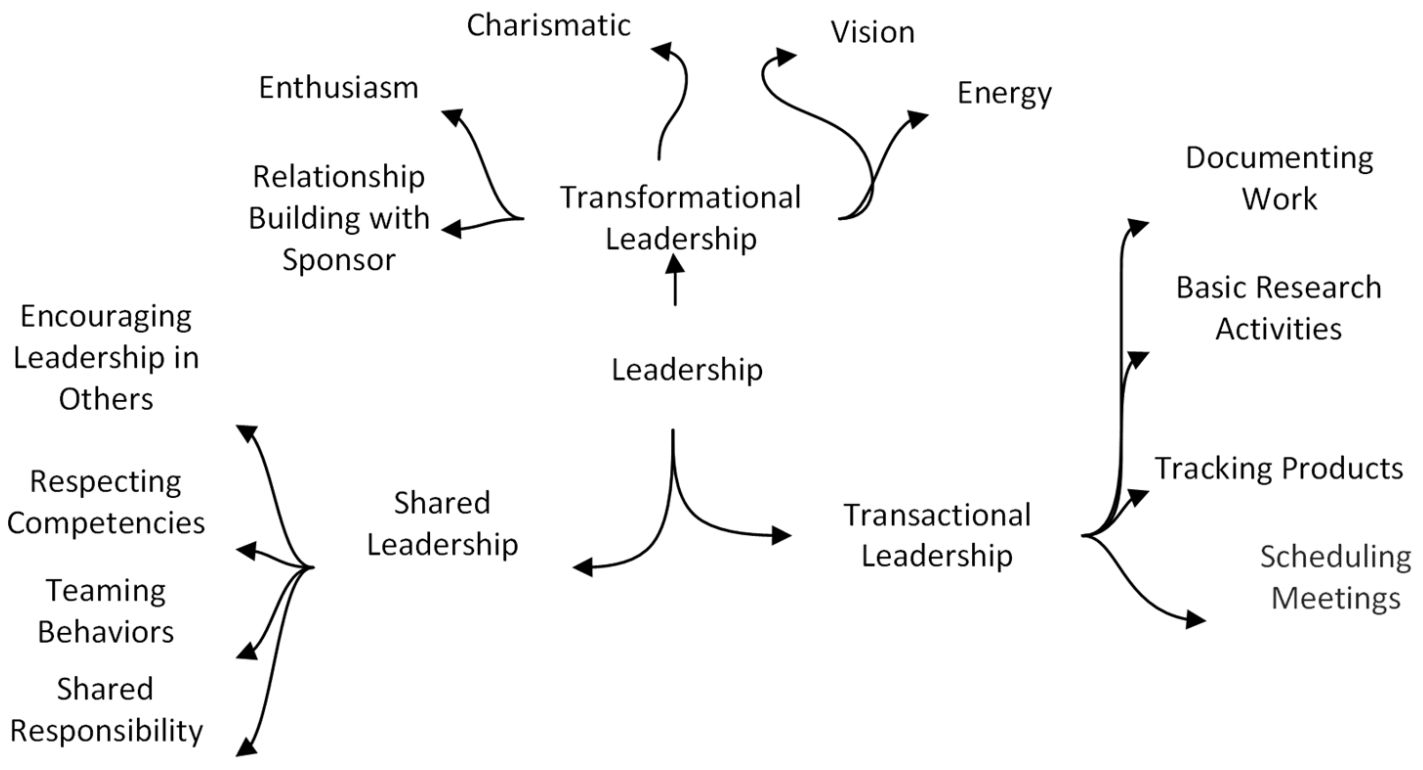

Figure 17. Exploratory concepts with the leadership subthem.

\section{Finding 1. Programs implemented a shared leadership.}

The first finding was that programs implemented a shared leadership model with transformational and transactional leadership occurring at all levels. In my research, the perception of leadership was critical to understanding how leadership manifests in a virtual scientific collaboration program. Consistent with Murase et al. (2014), I first looked at leadership in the context of focus, function, and forms of leadership. Qu et al. (2015) observed that when expectations for individuals working on a team included significant creativity in thinking, transformational leadership was positively related to the creativity of the follower. The DHS Centers of Excellence programs were targeted at creative solutions to complex problems. Consistent with $\mathrm{Qu}$ et al., nine of the participants described their program's top leader as having expressed behaviors consistent with 
transformational leadership. Specific participant responses regarding program leaders are provided here to support these observations:

- Participant A stated, “A lot of our team's attitude comes from the director's charismatic attitude. He has a really positive effect in terms of overall productivity and general enthusiasm for projects."

- Participant B shared that the top leader of a program was busy with relationship building with sponsors and university administration.

- Participant D described the top leader as "someone who knows everybody."

- Participant E shared that in a case where the leader was not viewed as successful; he did not have "that passion about this new area." Adding, "The government financial schedule and the universities financial needs do not align. Part of leading something like this [program] is being able to find ways through those challenges."

- Participant G shared that senior leaders had the responsibility of "justifying why a technical or certainly analysis method would be utilized. Initial motivation and justification came from team leads."

- Participant H had worked with multiple centers and noted that one of the very successful center directors was "very engaging and made you feel heard, even as a novice member of the team."

- Participant N noted that "we need someone with a vision for the center." Adding "Leader needs to be a scholar with an applied eye with lots of connections." 
Most of the participants expressed satisfaction with the top leader's level of charisma, energy, and ability to provide the program vision. Qu et al. noted that leaders with a transformational style allowed the follower to exhibit similar goals, values, and standards. The common goals, values, and standards appear to be consistent with an indication of shared leadership. Hoch and Kozlowski's (2014) provided strategies for enabling shared leadership that required accepting the new paradigm of shared responsibility, teaming behaviors, respecting member competencies, and encouraging leadership behavior in others. All participants interviewed expressed a program structure and expectations consistent with this paradigm of shared responsibility. It appears the shared responsibility behavior enabled organizations to function independently. Explanation of structure and impacts are provided in more detail under the program structure subtheme. Discussions with participants regarding shared responsibility are provided here:

- Participant A stated, "You don’t have to check with every member of the project on a weekly basis to make sure they are doing their work. By enlarge, everyone is self-motivated. There is no sense that you need to apply the screws. Everyone is interested in doing their work."

- Participant B shared the observation that technical area leaders were generally leaders in their field and usually busy with university obligations, oversight of postdocs, etc. Project leaders were quite busy with coursework and usually worked on more than one research project at a time. Graduate students were also an element of leadership in terms of getting the final report. "You are 
dealing with faculty members that don't have a lot of bandwidth and not terribly organized, to begin with." Noting further that "There were layers of leadership."

- Participant I noted that the development of ideas and leadership came from the researchers. "My department chair was the lead [for a technical area]. He collected together a few ideas that became our overall project area."

- Participant M reflected on the impact of the shared leadership model on communication. He stated, "I think the reason people default to conference calls is maybe a tendency for the lead PI not to want to be a dictator and to offer the opportunity for this big consortium to function as a cohesive unit and have everybody's opinion at the table."

One shared leadership model in the literature was the concept of agile software development. Agile was noted for formally introducing methods of communication, the frequency of communication, and tools for communication among agile teams (Highsmith, 2001; Denning, 2013; Ramos et al., 2013; Hilt et al., 2016). The shared responsibilities of an agile approach may apply to complex leadership collaborative challenges as well. Evidence of agile management behaviors was shared by participants on program structure and methods of communication. Specifically, the type and frequency of communication may be an indicator of an agile approach being implemented, either intentionally or inadvertently.

Another promising element of my research was the emergence of a concept encompassing early career researchers serving as transactional leaders. When asked how 
work was scheduled, tracked, documented, and overall brought together for delivery, all of the participants discussed the role and value of graduate and post-doctoral research support. These activities are consistent with Korman's (1966) description of initiating structure behaviors, later to be included in transactional leadership concept (Bass, 1997). The observations were early career researchers had more time available and were motivated to get research experience and publications. Motivation, combined with being less expensive and having a less financial drain on project funds resulted in these early career members of the team functioning as leaders. This concept was not a surprising finding within academic institutions, however, it was possible this reliance on the most inexperienced members of the team to fulfill a critical leadership role could be unique to an academic environment. A further discussion of findings related to academic environments was provided below under the research culture subtheme. This bottom-up option was not addressed in the extant literature and poses a potential area of investigation. It was also interesting to consider the low cost, highly educated, participants. This profile of participant needs be considered in any discussion of operating models or leadership training. Further discussion on the impacts related to structure, funding, and sharing research was covered under the program structure subtheme below. All of the participants addressed the importance of the graduate or postdoctoral researchers' role within the program activities. Highlights from the participant interviews noting the role of the early career members of the team are shared:

- Participant B discussed this subject at length, noting, "There was a succession of postdocs who would fill the role of being the point person and coordinator. 
If you were late, they would send you a reminder, and they would kind of pull that together."

- Participant B shared the observation that technical area leaders were generally leaders in their field and usually busy with university obligations, oversight of postdocs, etc. Project leaders were quite busy with coursework and usually worked on more than one research project at a time. The focused time and interest of early career researchers made their contribution significant within these program structures.

- Participant $\mathrm{C}$ noted, "We know they are not very strong skills in research, but they can do it." Emphasizing, "We have to integrate different levels of knowledge." "It is part of the capability building, part of the learning by doing."

- Participant D stated, "There is a difference in some ways in working with students. They bring you fresh ideas and youthful enthusiasm."

- Participant G emphasized, "Postdocs played a vital role. In that role, I interacted quite intensely with other postdocs."

- Participant F described the structure of project work as "a handful of graduate students and a few people in advisory positions."

- Participant $\mathrm{H}, \mathrm{K}, \mathrm{M}$, and $\mathrm{N}$ all noted that the graduate and postdoctorial research assistance did the bulk of the research work. 
- Participant I, “And at the funding level that I had, I basically got students together at my institution to work on it for their capstone project. I advised them we came up with some solutions."

- Participant $\mathbf{J}$ mentioned the relationship between funding and the use of students, stating "Those [programs] have the ability to fund students, and PhDs and masters programs, and postdocs, and that what's makes the next generation of product."

In discussions on the structure and various aspects of the vertical leadership approach of programs, all participants indicated the role and value of the early career researchers leading activities. These graduate and postdoctoral assistants were viewed as being the bottom of the structure, and viewed as critical to the success of the entire structure. Graduate and postdoctoral researchers had technical expertise, time, and motivation to operate as the driver for much of the work activity.

After considering the shared leadership evidence and role of the members throughout the structure of the programs, further analysis of the program structure itself was warranted. The shared leadership model combined with an understanding of program structure helps to provide a picture of the program environment and constraints.

\section{Subtheme 2: Program Structure}

Elements such as program structure, its implementation in a virtual environment, and its impact on participants were investigated during this data collection and analysis effort. Hoch and Kozlowski (2014) observed that the more distributed a virtual team was, the more its structural supports affected team performance. This impact extended to 
reward systems, available communication, and information technology. The structure of the programs described in the interviews was consistent across interviews. Figure 18 shows the four emergent topics from the program structure analysis. Observations related to the virtual environment expected within the programs are discussed in detail as part of the virtual environment subtheme below. Program structure has three elements including, funding incentives, project size, approach, and multilevel virtual structure. Each of these was additionally broken down into the associated concepts.

First, funding incentives resulted in competition rather than collaboration, which may have contributed to disciplines being distributed or separate. Second, project size was related to funding so larger projects might seemingly have more resources to include more participants. Third, the approach of research could range from basic exploratory research to applied research with a well-defined outcome. Finally, the multilevel vertical structure consists of a top leader who was usually titled the director. The director represented a single academic organization and was responsible for providing the vision, distribution of funds, and selection of the technical areas of the program. At the technical area level, a single organization was usually identified and offered a fair amount of autonomy to operate as a single team. Within a technical area, specific projects were distributed to individual researchers. In some cases, the researcher may opt to conduct the research themselves. Individual research was usually associated with very small projects. Most often a researcher engaged one or more graduate or postdoctoral research assistants to conduct the research, document the results, and write reports. 
Similar to the research offered by Wageman and Fisher (2014), I questioned if the leadership within the programs explored exhibited centralized leadership or multilevel vertical leadership structures. In order to conduct my analysis, I looked for evidence consistent with Wageman and Fisher's four areas of legitimate authority (a) executing tasks, (b) monitoring and managing, (c) designing the team, and (d) guiding overall direction. Exploration of the data indicated multiple individuals exercised each of the four areas of legitimate authority. Executing tasks was most commonly associated with the postdoctoral or graduate assistants, while monitoring and managing were generally associated with technical area leads. Designing the team and a portion of guiding the overall direction fell to the top program leader(s). Participants noted that direction was both a top-down and a bottom-up interactive process, with many ideas and suggestions coming from the early career members of the team. 


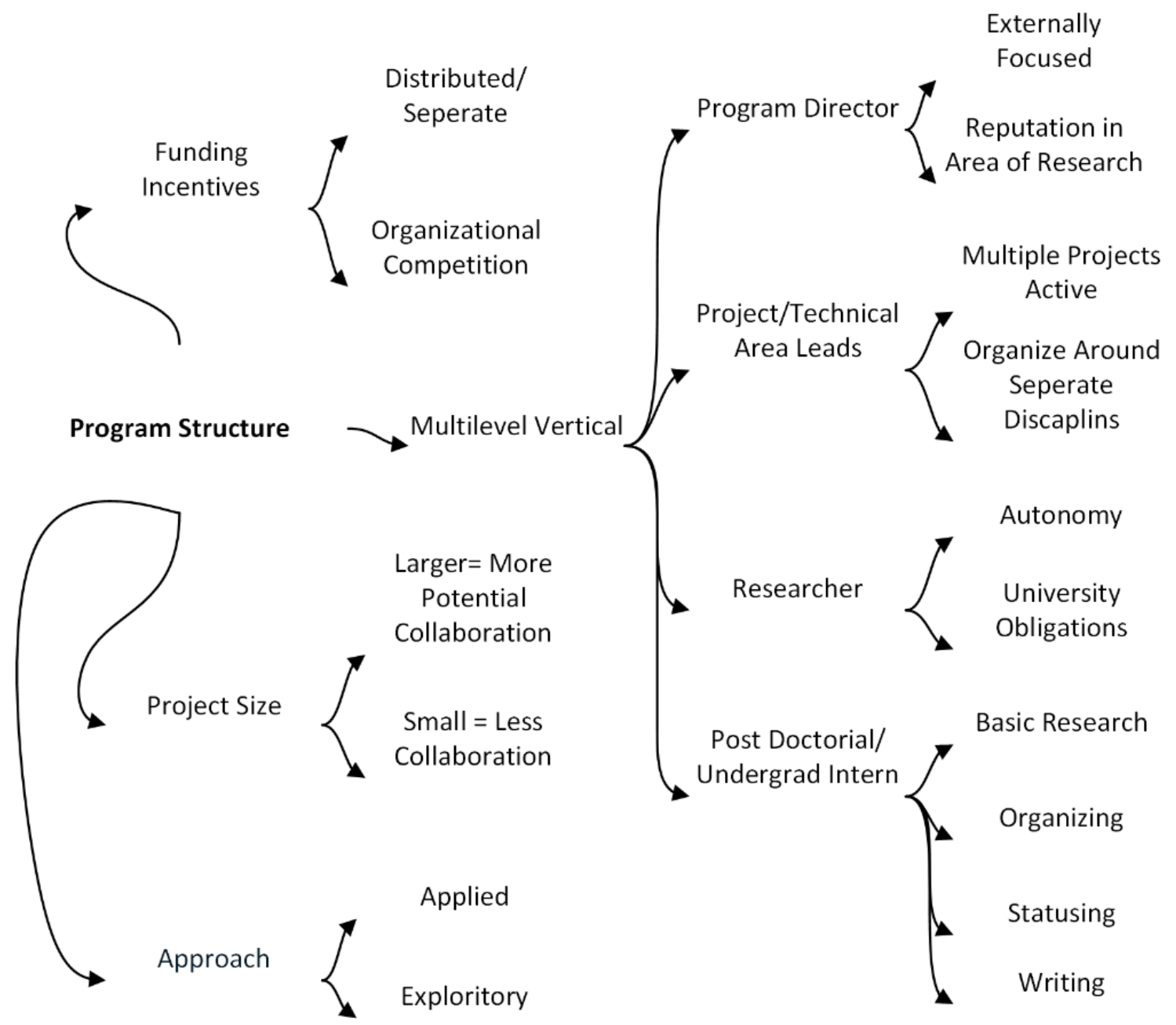

Figure 18. Exploratory concepts within the program structure subtheme.

\section{Finding 2. Programs focused on applied research.}

The second finding was that programs focused on applied research resulting in organizational structures segmented by discipline. One aspect surfacing as a theme in the data was the concept the requested research outcome of fundamental and applied research approaches. Fundamental and applied research may best be understood through the descriptions provided by the Congressional Research Services' (CRS) report for Congress regarding Department of Defense Research, development, test, and evaluation 
program (Moteff, 1998). The description grouped defense technology activities into seven areas. Basic research activities, listed as 6.1, were described as more fundamental research than supported new knowledge. CRS separated basic from applied research activities, which were listed as 6.2 within the development, test, and evaluation program. Applied research activities included exploratory development generally with existing technologies or new research with a specific mission. The remaining five activity categories took the technology through design, development and fielding of technologies. For this discussion regarding my observations in the data, basic and applied research were used consistent with the CRS descriptions. The impacts of DHS Centers of Excellence program research focus was noted by the participants and appeared to impact the level and type of collaboration experienced.

Participants observed a structure and research approach often guided the option of collaboration with other researchers and organizations. In one instance the researcher was the only person working a particular project; in another, the project research team was a group of individuals within the participants' own organization. In another situation, the researcher reached out to multiple researchers representing different organizations. Exploration of patterns in researcher experiences was one element of my analysis.

To further understand the drivers for this diversity in collaboration during the interview, probing questions were used, such as, what determined the collaboration within your project area? These probing questions resulted in a variety of responses leading to the distinction in my research on funding method, project size, and the research approach. The funding approach could include larger cross-discipline distribution 
intended for organizations to collaborate, or small one or two researchers and a handful of graduate and postdoctoral research assistants. The funding approach was also discussed regarding the 1-year funding allotments and expectation of product delivery on a quarterly basis. Project size was related to funding in that some larger projects would have more funding associated with the work, and therefore more participants. The smallest projects discussed by participants included one researcher with one or two graduate or postdoctoral research assistants. The research approach was related to the expected outcome of the investment. Participants discussed fundamental research as being exploratory in nature with outcomes adding knowledge to the scientific community. Many of the participants discussed program outcomes as being applied to a specific national security problem and much further along the concept development spectrum towards implementation by DHS. Participants noted the relationship of research approach guided by the outcome, funding, and project size. Specifically, they discussed the frustrations with operating on an annual award basis, the small size of funding allotments, and the impacts on collaboration opportunities:

- Participant F observed, "funding influenced the direction that [we] went."

- Participant $\mathrm{G}$ pointed out that collaboration depended on the project. If it was small, it could be done by a single researcher. Alternatively, if the project was large or complex, it may require a larger more coordinated team.

- Participant I, “So it's this annual competitive sub-award process basis for the research." 
- Participant J agreed that size guided the level of collaboration and felt the awards were small, stating "It's not a largely funded activity for DHS relative to other things."

- Participant K and I discussed the short durations of funding. K stated, "The types of research sponsored by the centers is very short term, and it is very much oriented on immediate results, and it's not allowing the breadth of fundamental research.” Where I noted, "The center leadership knows how to smooth out those [annual funding] bumps."

- Participant $\mathrm{M}$ and $\mathrm{N}$ emphasized the importance of funding to researchers, $\mathrm{M}$ noted "faculty and researchers are always looking for funding." Participant N warned 'Don't underestimate the money part. Most university people get nine months of funding from the university, so they are looking for summer funding."

The structure shared by participants when describing their programs resembled the silos discussed by Kuhn (2012). Kuhn (2012) noted that academic departments behaved more as competitors rather than collaborators. He maintained that professional incentives promoted a competitive environment when organizations began to compete for research and funding. Years of competing for research dollars resulted in organizations striving to distinguish themselves from each other (Sanberg et al., 2014; Walsh \& Huang, 2014). In general the participants did not discuss this silo effect in terms of competing departments. The silo effect was discussed by more than half has a factor in 
collaboration. Participants noted some competition, but also noted that collaboration, or coordination, was a competitive advantage:

- Participant B It is just a competitive environment that anything that makes the proposal more competitive will be done

- Participant D noted, "The structure tends to be defined by the contractual arrangements with a prime contractor and subcontractors." Adding, "The researchers usually shared a discipline, but brought different experiences to the research." This is consistent with Participant C's observation that the program was a "research unit structure" that "integrates different levels of knowledge."

- Participant $\mathrm{H}$ described the projects has having a "tendency to be split up by discipline.” Adding, "I would say one of the partners was probably more motivated by the piece of pie available to him than the work that needed to be done."

- Participant I, J, and K discussed the tension between collaboration and competition. Participant I noted "If you want to be competitive you have to collaborate." J agreed, saying the "concept of the competition for funding is obviously an element in the idea of collaboration and cooperation. In the DHS Centers, when you were going through the process of getting your piece of the project, the process cooperative when you're in a competition." Participant K summed the environment this way, "when there is a call for new projects, 
there is still a little bit of competition between these people for the same call for projects, so it's collaborative and competitive at the same time."

In addition to challenges with smaller funding amounts, which appeared to be a factor encouraging single researcher activities. It was possible that the applied nature of DHS Centers of Excellence programs was a factor in both enabling and encouraging the separation of organizations and disciplines. The distinction in a research approach as a continuum from basic fundamental to fully applied research was consistent with Bogers et al. (2017), who described a similar continuum starting with the intra-organizational approach and extending to the concept of industry-wide approach. The DHS Centers of Excellence programs appeared to be more aligned with Bogers extra-organizational approach, rather than the inter-organizational level of analysis and research. Extraorganizational meant the programs had external stakeholders and functioned as individuals operating within a community. By contrast, inter-organizational analysis and research included alliances, networked interaction, and an ecosystem approach (Bogers et al., 2017). Participants discussed their research activities as more coordinated and extraorganizational than fully collaborative:

- Participant A, D, and M discussed their respective independent research activities, asserting "All I have to do is make sure the work that gets done meets certain criteria." D noted, "It [the research] is generally broken down, you do something, and I do something, and in some period of time we will sync up.” M stated, "There's not a lot of collaboration or integration across projects." 
- Participant B had a similar experience describing his interaction with other researchers as "they were going first and publishing, then we were coming along and using their information in our models."

- Participant I and $\mathrm{K}$ agreed that the program itself was organized for coordination of independent efforts, stating "the executive leadership is basically focused on coordination of the team." With K explaining, "It's really a center which integrates independent researchers into research teams, and they do report to the center, and the center reports to the sponsor, but there is not much cross-pollination between the research teams within the center. From my experience in more than one center, both DHS centers have been operating in the same organizational mode."

- Participant L provided insights into the concept of coordination and collaboration in his description of interdisciplinary work (more collaborative) and multidisciplinary work (more coordinated). L had this to say, "Sometimes the question or problems are complex, and you realize that it can't be done within a single discipline. So you want to bring in other disciplines and other people, other experts in the hope that they're going to be able to provide a larger field of expertise to bring to whatever the question you have. If it stops there, we're talking about a multidisciplinary research effort. Here you have a bunch of people. Multiple disciplines, sitting in a room being told, hopefully, that there is money for this project, find some aspect of it that you can do. That is multidisciplinary research. The approach can be formulated by the 
people sitting in the room, or it can be formulated by the people who are organizing the meeting, but that is multidisciplinary. Many strands that have to be woven into something. Or else it's just independent strands"

The research approach appears to be a consideration when exploring the drivers for collaboration or coordination. It was apparent in the centers that coordination was more frequently used than fully collaborative efforts. Several participants indicated that the more applied research approach drove a multidisciplinary, but not necessarily interdisciplinary, research relationship among the program participants. The focused application of the DHS Centers of Excellence program investment may be one reason for a more coordinated result. Participants shared their perspectives on the applied nature of the DHS programs:

- Participant D "There was a huge motivation to get something that just worked. You're putting a system out there and this was different than a lot of research that is just trying to demonstrate a concept." Participant K The time to technology transition and adoption by DHS is going be very short.

- Participant $\mathrm{F}$ and $\mathrm{O}$ discussed the attraction to the programs because of their applied nature, F stated, "That it seems to be somewhat more tangible and rewarding to put in someone's hands. As opposed to maybe some of the more theoretical that maybe not guaranteed to work or maybe there is no deadline for that. That's a little bit scarier to me. I like to see the end." Participant O attributed himself by saying, "I'm more on the applied end of the spectrum." 
- Participant $\mathrm{J}$ discussed the challenge of trying to deliver a targeted product while conducting basic research. She noted, "some of the [organizations] are doing some research around a basic type research, and are in general doing some things that support the mission. But at the end of the day, all of those products are not as tightly knitted together as some of the more operational organizations."

- Participant $\mathrm{N}$ discussed a transition over time of the program from more exploratory to more applied research. He described the process as "In the first years we were essentially testing out our tools and methods. While it was problem focused, it was really very fundamental because we didn't know if any of those tools and methods would work" Later adding his "[recent research] was definitely applied research and was bordering consulting."

I observed a pattern in the program structure of DHS Centers of Excellence programs and its relationship to funding methods, project size, and research approach targeted at more applied outcomes. The influences of program structure and the virtual environment of multiorganizational research and development programs guided the insights I drew on how these structures look to the project participants.

\section{Subtheme 3: Virtual Environment}

Wadsworth and Blanchard (2015) maintained that virtual team leadership as a process was very different from face-to-face leadership. They noted virtual leaders needed to spend more time in contact with team members, and highlighted technologies and characteristics of successful virtual leaders. Consistent with Walsh and Huang's 
(2014) findings, early and late stages of project work were described as having more communication. Figure 19 provides a graphic of elements found within the virtual environment for my research. Communication types and methods were considered including face-to-face and technology options. Also, participants discussed the number of participants, duration, and frequency of communication.

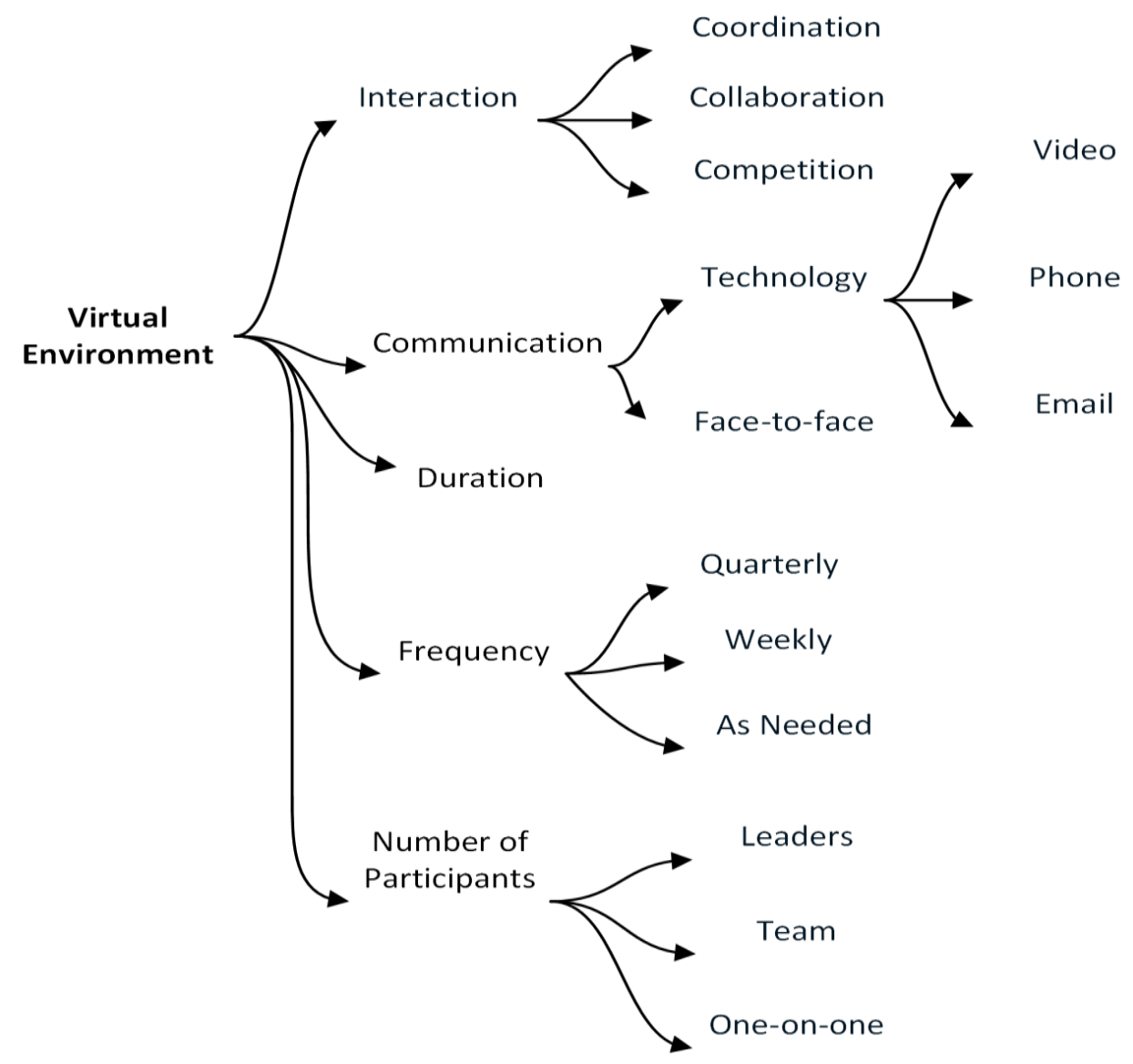

Figure 19. Exploratory concepts within the virtual environment subtheme.

In figure 19, communication included face-to-face and technology options, such as e-mail, phone, and video conferences. The number of participants, duration, and frequency, appeared to be related to larger meetings 
occurring at the beginning and end of the research and ongoing one-on-one meetings on a weekly basis.

\section{Finding 3. Coordination versus collaboration within programs.}

An important finding in my research was the preference of coordination over

collaboration behaviors between partners when considering the collocation versus virtual nature of the program participants. Collaboration occurred within collocated teams and coordination occurred between virtual partners. Participants noted that most of the time, they worked with other individuals within the same organization, and often located in the same building. Coordination took place across and between organizations where individual collaborative subteams reported the status of work through hierarchical process regularly (generally weekly). Agile teaming was noted for formally introducing methods, the frequency, and tools for communication among agile teams (Highsmith, 2001; Denning, 2013; Ramos et al., 2013; Hilt et al., 2016). Potential evidence of agile management existed in the data. Weekly program meetings to reporting status and the eventual coordination of research toward a final overall program product provided in the form of an annual report were discussed as common activities within the program structure. Ten of the 15 participants interviewed described a weekly status meeting to coordinate independent research activities across the program.

- Participant $\mathrm{C}$ shared that "we have a two-hour weekly meeting with all the scientific team. This was very important." "Everybody is in the room except one or two." "Here in this particular center, we are 10-15 people every week 
for 2 hours together in the same room and 1-3 connected by [conference line]."

- Participant D stated that the communication was "weekly or monthly" with work broken down.” Adding his opinion regarding virtual forms of communication, "When you have positive things to say or normal business interactions, the phones and web conferences work fine. If you have a disagreement with someone, I find that harder to deal with on the phone. You can't see a person's reaction."

- Participant F also experienced a "weekly meeting where the group of folks in the same organization would get together and talk about the project they worked on."

- Participant $\mathrm{G}$ experienced some multiorganizational and virtual project teaming; he noted however that "As the project came to an end, [they had] a lot more person-to-person interactions."

- Participant H, I and $\mathrm{O}$ all described weekly meetings run by the program director.

- Participant M was surprised by the weekly calls when he join a center, noting "PIs have to participate in this weekly conference call." Noting, that "initially I had no expectation of weekly conference calls or really say collaboration across the center."

Technology did not surface as important in program coordination because e-mail and phone calls were sufficient for coordination and face-to-face meetings most often 
served for connecting. According to the participants interviewed, the organizationally separate structure of the project teams within a program was often determined by proximity, common technical interests, and organizational alignment. This coordinated rather than collaborative environment was notable, given the genesis of the programs by the U.S. Congress expressed the multiorganizational collaboration expectation. This result also lends itself to further investigation into the nature of scientific collaborations under which these programs existed. Eleven out of the 15 participants provided insights on the cooperative nature of the DHS Centers of Excellence programs:

- Participant $\mathrm{G}$ described the final report as a coordinating factor, noting "We were aware of their tasks, as we progressed and the outline of the overall report started coming together." Participant K was also not heaving engaged with others whole conducting her research, describing it as "not necessarily seeing others' researchers who are doing research within the same center. But the monthly collaboration is basically coordination on your own task." Participant M differentiated the Centers of Excellence programs from other types of research he conducted. "This project is different from other projects I've been on in that each PI is responsible for a topic that fits within this umbrella of the Center's vision but can be very distinct from other topics funded by the center."

- Participant $\mathrm{H}$ observed an advantage to virtually independent teams noting, "Not having them collocated meant that our lead had more discretion over the direction of the work, pace, schedule, all these aspects of the project." 
Management responsibility for virtual coordination in these DHS Centers of Excellence programs may resemble a market-based partnership. Du, Lenten, and Vanhaverbecke (2014) described two types of innovated projects, science-based and market-based. The concept of science-based appears to be aligned with fundamental research. Likewise, the concept of market-based appears to be aligned with applied research. Du et al. provided a science-based model characterized as inexpensive, lower risk, and a good source of specialized knowledge. Market-based partnering was characterized as involving the customer, drawing on industry-specific expertise, and working as a community of practice (Du, Lenten, \& Vanhaverbecke, 2014). The formal management structure and customer involvement of DHS Centers of Excellence programs appear to be more closely aligned with the market-based partnering described by Du et al. (2014). Participants discussed the engagement of actual users, discussing both challenges and advantages given the specific mission outcomes associated with individual DHS Centers of Excellence programs:

- Participant E felt stringing that "The center had a real impact in terms of academics, in terms of tool creation, in terms of forwarding the science, and also applying it to DHS mission."

- Participant I explained how the mission objectives are connected to the research. "They had a series of unifying field tests. And those were intended to be unifying scenarios, so as a center came up with these scenarios working with stakeholders that DHS prompted them to work with the center." 
- Participant $\mathrm{J}$ had a warning, "I would add though. If we continue to only focus on the very applied end of the spectrum, then we are going to miss getting that very seed corn. That part of what we do that plants the seeds. If you're not developing that seed corn, then you're not going to have things that grow out 5 years from now, that might then move into a more applied nature. I really think there should always be room for basic research inside a research agenda, and the focus for DHS was very focused on the end users, but there needs to be some room for basic research. We still need the basic investments to help us start some ideas that we couldn't imagine.

- Participant $\mathrm{K}$ and $\mathrm{N}$ genuinely enjoyed the bridge into applied research, $\mathrm{K}$ noted "I find from my own experience that working with industry and working with DHS gives you a unique perspective on your research where you get the use cases, you get the problems which are relevant for the application of your research, and that is invaluable for publishing, for your own research growth, and that is the motivation for the faculty." $\mathrm{N}$ felt similarly motivated, noting "I personally am motivated by problem-solving and so are many others. The notion of working on an interesting problem, even if it's sort of applied work, there are always challenges."

I used project management formality as an indicator of project partnering to further understand the potential meaning of the participants' experiences within DHS Centers of Excellence programs. Du et al. (2014) evaluated project management formality as it related to science-based versus market-based partnering. They concluded 
that market-based partnerships required a more formal project management structure. Working backward from their finding, the formal hierarchical management structure described by participants provided evidence of a coordinated, but less collaborative environment. Several of the participants indicated that formality was notable in the DHS Centers of Excellence programs. The programs also were targeted and often had active participation by the planned customer, a characteristic of market-based partnering according to Du et al. (2014).

The observation of virtual leadership following coordination rather than collaboration was valuable to understand the organization, management, and expectations within DHS Centers of Excellence programs. The programs function with academic organizations operating with collaborative scientific culture. Further investigation of the data related to scientific collaboration may illuminate further the following research culture subtheme.

\section{Subtheme 4: Research Culture}

The DHS Centers of Excellence program research culture could be explored through the lens of collaborative science (Leiserson \& McVinney, 2015). The study of collaborative science research acknowledged cultural factors influencing successful teaming, and considered the effects of competition for funding. Dailey (1978) highlighted the culture of scientific research and challenges to collaborative problem-solving. Challenges continue to emerge in scientific culture with more teaming environments emerging through open science in competing organizations (Walsh \& Haung, 2014). Some researchers have taken on the scientific culture challenge to collaboration by 
focusing on investigating the challenges of multiple departments in educational institutions ( $\mathrm{Su}, 2014)$. In my study, I considered the research culture and highly educated environment of the research and development teams through viewing them as scientific collaboration programs.

Specifically, this research culture stems from having a research environment within an academic setting. Shrum et al. (2007) included in this culture the competition for funding and prestige discussed by Kuhn (2012). In addition, Shrum et al. included elements such as the scale of collaboration, the organizational structure of collaboration, the technology used, the information interdependencies, and the collaborative relationships. Figure 20 lists the elements associated with research culture. Motivation, relationships, and prestige were all factors in collaborations discussed by participants.

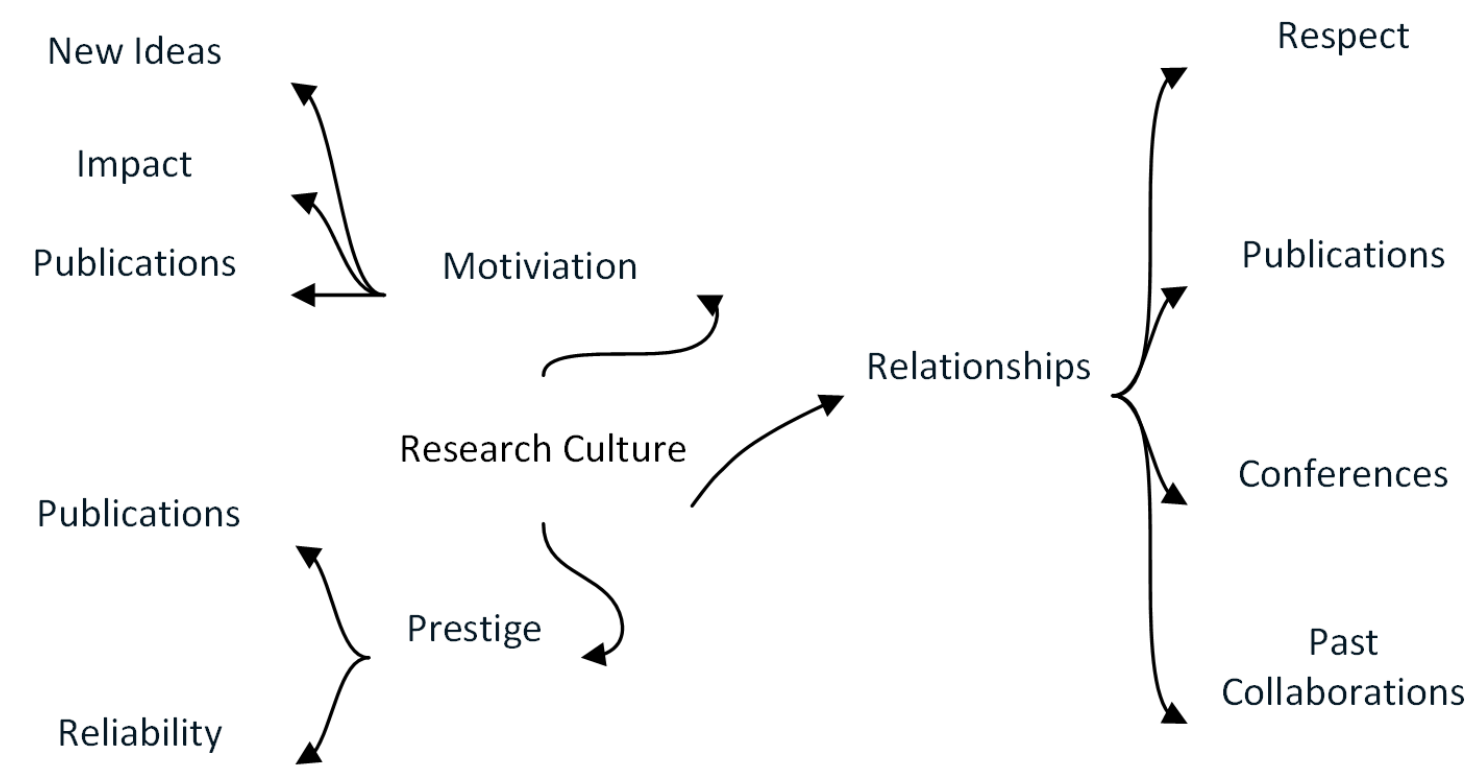

Figure 20 Exploratory concepts within the Research culture concepts.

Finding 4. Program members were primarily self-motivated. 
The forth finding was that program members were primarily self-motivated with publishing serving as evidence of respected behavior. Walsh and Huang (2014) researched collaborating with American and Japanese scientists facing the challenges of collaborating in a competitive scientific environment. In Walsh and Huang's (2014) research on entrepreneurs in scientific research organizations, the behavior of publication secrecy among collaborators affected the willingness to share results. However, publication secrecy was not observed in my study. One metric relating to Belderbos et al., (2014) was the incentive to publish. The incentive to publish was a measurable way to produce and share scientific knowledge. Participants were consistent about the opportunity to publish. Twelve out of the 15 participants interviewed discussed publications specifically as a key motivational factor. Half of the participants noted the closely related concept of impact. The three participants who did not explicitly discuss publishing noted that they were interested in producing a product, but not necessarily a journal article or research paper. The applied nature of the DHS Centers of Excellence programs lends itself to a product-oriented impact. Participants shared their interest and motivation for publishing their work:

- Participant A described the motivation as a "nerdish academic affinity toward research," with, "everyone interested in what they were doing." "I think as long as I was doing interesting research, the opportunity for publications, and producing high-quality products, I think I would be fine." 
- Participants B, C, E, and I noted publishing was the main reason for early career members to lead. Participant $\mathrm{H}$ described her role as a new researcher as "important for publishing."

- Participant $\mathrm{C}$ described publishing as a scientific responsibility of sharing findings. Noting that it was "mandatory to publish. You have to publish." Similarly, Participant F noted, "Publication was very much encouraged."

- Participant E put it this way, "the institutional culture is an important factor." Adding, "Much like people have personalities, institutions have personalities as well.” Participant J also considered the institutions perspective, noting "Look at the 10-year tract requirement. You have to have so many publications, so many research grants, so many classes, and so many students."

- Participant $\mathrm{G}$ and $\mathrm{H}$ felt that publishing was expected and guided their work so that "An article came out from each of the pieces. That was quite rewarding. We used to publish, coauthor and publish in academic journals."

- Participant $\mathrm{K}$ again noted the unique perspective gathered when working on mission-specific research, observing that "working with DHS gives you a unique perspective on your research where you get the use cases, you get the problems which are relevant for the application of your research, and that is invaluable for publishing." 
- Participant M noted the use of published material in learning about other researchers, stating "Reading journal literature is one way to identify experts in the field."

Specific to organizational incentives, Lee et al. (2015) found that organizational performance appraisal systems needed to match the preferred collaborative attitude for a follower to engage the leader as expected. The collaboration was encouraged, but primarily within the same institution. Jay (2013) noted that multiorganization entities required new organizational success criteria. There was no evidence provided by participants indicating novel or unique organizational incentives were in play in these programs.

Another common observation was the role of scientific prestige. It appeared that the reputation of the scientists involved played a key role in the program partnering and coordination efforts. First, during the proposal phase of the research. Scientific prestige and publications were noted as helping to make connections between researchers and add strength to a proposal. The second point in a program when reputations were depended upon heavily was in later stages of the project coordination when deliverables came due. Reputation was perceived to be a reflection of the reliability to deliver as agreed. The multiorganizational nature of the program team was most notable during these moments of signification coordination, observed in the early and late stages of a program's project work. Participants discussed this phenomenon:

- Participant B noted that in addition to publishing evidence, relationships and prestige were spread by "word of mouth." 
- Participant D noted that the most intense collaboration occurred during the proposal phase of the work. Adding, "you tend to get motivated by deadlines."

- Participant E noted that when there was no obvious place to publish, "We worked with a professional society to start a symposium and then a conference. That gave the researchers a place to publish their work."

- Participant $\mathrm{J}$ took a practical approach when discussing motivation, emphasizing "I'd like to say it was all altruistic, but it is part of my business. For the success of the center, they have to deliver."

- Participant $\mathrm{K}$ and $\mathrm{N}$ bought the additional expectation that self-motivation may be a sign of maturity, and that "The only types of faculty who can participate in these centers are mature faculty, experienced faculty, who have multiple sources of funding." Participant N, extended that experience to the lead of the center, noting the lead needs to be "somebody who has both the scholarly credentials and the connections with the client base."

As I noted above, external coordination at the program level was often a result of past research engagements. Previous experience with a partner resulted in increased knowledge of another researcher's skillset and an increased level of trust. Trust was a key component of previous research on collaboration (McNab et al., 2012). Trust may have been a result of the participant's relationship. The participants most frequently used the concept of respect to describing their relationships with fellow researchers and leaders. Respect appeared to stem from demonstrated self-motivation as described by participants: 
- Participant A and M noted the expectations that researchers would behave professionally in delivering their work. Participant A noted, "There is no sense that you need to apply the screws. Everyone is interested in doing their work." Participant M suggested that over time you will know how a researcher performs. Explaining that initially "You really have to fund the people who will do good work, and then you have to trust them to do it."

- Participant B chose collaborators based on individuals he had worked with at a previous university. Participant E also found previous relationships to be a benefit in their noncollocated example of collaboration. "They were not collocated; however, one of the professors came from the other university." Noting also that relationships were built through student exchanges and internships with other organizations."

- Participant C “you don't have to be collocated. Communication is number one. You always have to communicate. It is online. When I was in the field, the first thing I did was install an antenna because we need to communicate."

- Participant $\mathrm{J}$, and L both discussed trust building. $\mathrm{J}$ noted, "I think it is a trust relationship between the sponsor and the center." With L adding, "That is where a center is able to be positioned with success, experience, and trust." There was not enough information from participant interviews to go into the number of interactions and trust relationship. These relationships may warrant additional investigation by future researchers to understand the relationship between virtuality and the number of participants engaged in a research project. 


\section{Summary}

The purpose of this qualitative case study was to understand followers' perspectives regarding virtual leadership within complex multiorganizational scientific collaboration programs. I characterized successful leadership of complex multiorganizational scientific collaboration programs as fostering creativity, crossing organizational boundaries, encouraging interdisciplinary collaboration, and providing customer-driven solutions. These characteristics were noted as goals for the DHS Centers of Excellence programs (U.S. DHS, 2017).

I posed a single overarching research question: What virtual leadership strategies do leaders of interdisciplinary collaborations use to motivate their highly educated scientists across organizations? I broke down the question into four subthemes: leadership, program structure, virtual environment, and research culture. A qualitative design was used to address the gap in extant research on virtual leadership in multiorganizational scientific collaborative environments. Previous researchers called for a more holistic consideration of leadership of complex multiorganizational systems (Dinh et al., 2014; Henry, 2015). I examined leadership of these complex scientific collaborations from the followers' perspectives.

During the interview process, demographics questions were asked to understand the participant's role in the program. Information was collected for each participant regarding discipline, highest degree achieved, and the number of years the participant had been a researcher. The role(s) of each participant discussed to understand if the 
participant had experiences as a program director, technical lead, or early career researcher.

Participants were then asked to describe the program structure including the leadership in the program. For this study, I was seeking the follower perspective to learn more about leadership strategies as viewed by their program members. I asked probing questions regarding their participation in the program, whether leaders were collocated if the participant was in the same organization as the leader, and whether they shared the same discipline. In general, the participant's input was consistent with a shared leadership model.

Participants were asked about their roles and what leadership if any, they demonstrated during their time as a participant in the program. Again, probing questions were used to learn how participants typically interact with leaders, frequency, mode, and formality. The participant's input was a mix, depending on the leader they were discussing. In the cases of interactions with the director or technical leads, the interactions were usually in person, monthly, and were deemed status meetings. The interactions with early career leaders, such as graduate and postdoctoral research assistants, were done primarily by face-to-face and through e-mail with weekly meetings to status work and shared interim findings. The discussions on interactions highlighted the distinction in experiences of participants between program level coordination efforts and individual project level collaboration efforts.

Additional open-ended questions were asked regarding leadership strategies observed or any recommendations to improve future interactions. Specific impacts 
regarding the collocation or lack of collocation were discussed. Specifically, charismatic leadership was discussed in this portion of the interviews. Participants used similar wording to describe the visionary responsibilities of the director, in addition to relationship building with sponsors. Providing the flexibility to conduct impactful research was a common theme, and viewed as successful.

The final set of questions for each participant were related to motivation. Each participant was asked to think about the kinds of activities, incentives, or behaviors brought out creative interdisciplinary teamwork and new ideas. Participants were asked about motivating factors and asked to provide scenarios where they felt motivated. The motivational factors for participants were the ability to conduct research that made a scientific impact and was sufficient to allow them to publish their results. Significant selfmotivation was common and enabled noncollocated subteams to operate independently, yet coordinated, with the larger program. Leadership behaviors facilitated this opportunity and removed barriers, such as funding, communication, and politics contributed to researcher motivation.

The study results were binned into four subthemes: leadership, program structure, virtual environments, and research culture. Each of these subthemes had an associated finding consistent across most, if not all, of the participants interviewed. In some cases, the findings reinforced observations made by earlier researchers interested in similar areas of research, such as the value of transformational leadership in creative environments. In some cases, observations and experiences shared highlighted new areas 
of research. For example, the difference between coordinated research and collaborative research and the impacts on virtual leadership expectations.

Chapter 5 provides further discussion on the implications of my research. Summaries guiding future investigations into virtual leadership within complex multiorganizational scientific collaboration programs are provided. Limitations and recommendations provide a basis for thought and a path forward for this management and innovation research area. 
Chapter 5: Discussion, Conclusions, and Recommendations

The purpose of this qualitative case study was to explore complex multiorganizational scientific collaboration programs through understanding followers' perspectives regarding virtual leadership. Successful leadership was described as fostering creativity, crossing organizational boundaries, encouraging interdisciplinary collaboration, and providing customer-driven solutions (DHS, 2017). I used a qualitative case study to represent the collection of U.S. DHS's Centers of Excellence programs, interviewing 15 individuals from 10 programs.

I identified four findings when asking what virtual leadership strategies leaders of interdisciplinary collaboration programs use to motivate their highly educated scientists across organizations. Finding 1, within the leadership subtheme, displayed how programs implemented shared leadership. Finding 2, within the program structure subtheme, demonstrated that programs focused on applied research resulting in organizational structures segmented by discipline. Finding 3, within the virtual environment subtheme, showed collaboration occurred within collocated teams and coordination between virtual partners. Finding 4, within the research culture subtheme, illustrated how program members were primarily self-motivated with publishing serving as evidence of respected behavior. Targeted training consistent with these findings could lead to positive social change through preparing future scientific leaders for virtual and interdisciplinary collaborations.

After identifying a gap in research on virtual leadership in scientific collaborations, I designed a qualitative study to seek a more holistic consideration of 
leadership in complex multiorganizational systems. Through my research, I gained an enhanced understanding of the program team dynamics in interdisciplinary collaborations of highly educated individuals. Researchers indicated self-motivation as the most critical factor in their research environment. They also agreed the ability to conduct impactful research was important. The understanding of impact and its relationship to the DHS Centers of Excellence missions was part of this research analysis. Leadership behaviors could facilitate the opportunity to conduct impactful research and remove barriers such as funding, communication, and bureaucracy were deemed motivational. Participants in my research discussed a shared leadership model with an explanation of the key roles of graduate and postdoctoral research assistants. I observed a pattern of participant distinction between coordinated and collaborative research. There appeared to be an impact on the participants' choice of coordination or collaboration based on the virtual behaviors of the research teams and their expectations of virtual leaders. This chapter includes a discussion of the findings, limitations, recommendations for further research, and implications of my research study.

\section{Interpretation of Findings}

My research was guided by a single overarching research question: What virtual leadership strategies do leaders of interdisciplinary collaborations use to motivate their highly educated scientists across organizations? Findings discussed in this section were interpretations drawn by me, as the research instrument, and gathered from the exploration of results from semistructured interviews with program members. I drew insights regarding the participants' views on leadership within virtual multiorganizational 
research and development programs, program structures and the potential impacts of those structures, teaming within an academic environment, and finally, aspects of research conducted within virtual team environments. Overall, I learned about the participants' views on leadership, program structures, virtual environments, and research culture. A key finding emerged under each one of these four subtheme areas.

\section{Subtheme 1: Leadership}

Finding 1. Programs implemented shared leadership. The first finding was that programs implemented a shared leadership model with transformational and transactional leadership occurring at all levels A clear leadership element that emerged when analyzing the data for my research was the evidence of shared leadership. Hoch and Kozlowski (2014) provided strategies for enabling shared leadership require accepting the new paradigm of shared responsibility, teaming behaviors, respecting member competencies, and encouraging leadership behavior in others. Figure 12 showed that in addition to the idea of encouraging others beyond the program director to express leadership, the evidence of paradigm of shared responsibility also exists within these programs. All participants interviewed expressed a program structure and expectations consistent with this coordinated work paradigm of shared responsibility. It appeared that the shared responsibility behavior enabled disparate organizations to function independently. I conduct further analysis of the structure and impacts in more detail below.

Management responsibility for virtual coordination in these DHS Centers of Excellence programs may resemble a market-based partnership. Du et al. (2014) 
described a market-based partnering characterized by involving the customer, drawing on industry-specific expertise, and working as a community of practice. The formal management structure and customer involvement described by the participants of DHS Centers of Excellence programs appear more closely aligned with the market-based partnering.

A kind of shared leadership, which may be unique to an academic environment, was early career leadership. I define early career leadership as expressed leadership behaviors in the most inexperienced participants (graduate and postdoctoral researchers) within the program. When asked probing questions about how ongoing work was scheduled, tracked, documented, and prepared for delivery, all the participants discussed the role and value of graduate and postdoctoral assistant support. This concept of putting transactional leadership responsibilities with the most inexperienced members of the team would be a point of divergence from other collaborative environments. The medical industry was an example of an industry in which researchers have begun to evaluate the benefits of interdisciplinary cooperative environments (Manusov et al., 2015; Lalor et al., 2013). Lalor et al. (2013) focused on multiple disciplines in a single organization in his review of different levels of participants in interdisciplinary medical teams. Bedwell et al. (2012) analyzed the various uses of the concept of collaboration across many disciplines. They determined that collaboration can serve as a higher-level construct housing ideas of cooperation, teamwork, and collaboration. Poirier et al. (2016) began the extension of collaborations and innovation into the architecture, engineering, and construction fields. In their research, Poirier et al. noted that collaboration was not specifically defined and 
was approached differently by various researchers. In these various examples of collaborations, transaction leadership by early career participants was not discussed. All participants discussed early career transactional leaders and their role indicating that graduate and postdoctoral research assistants were a key part of these complex programs.

The participants' observations were current and past graduate and doctoral students had more time available and were motivated to obtain research experience and publications. Motivation, combined with being a reduced financial burden on project funds resulted in these early career members of the team functioning as transactional leaders. This bottom-up option was not addressed in the literature and poses a new area of investigation. It was possible this finding may only be a phenomenon in academic environments. A further discussion of findings related to academic environments was provided below under the research culture subtheme.

Hoegl and Muethel (2016) noted in their research on distributed teams that there was great value in shared leadership, yet that value can remain unrealized if the leader remains tied to traditional leadership models, demonstrates overconfidence in their leadership role, or fears becoming unessential. They also provided strategies to enable shared leadership such as shared responsibility, teaming behaviors, respecting member competencies, and encouraging leadership behavior in others. Qu et al. (2015) observed that when high-creativity expectations were present, transformational leadership was positively related to the creativity of the follower. The DHS Centers of Excellence programs were creative solutions to complex problems. Consistent with Qu et al., seven of the participants described their program's top leader as having express behaviors 
consistent with transformational leadership. Consistent with Hoegl and Methel, 10 of the 15 participants mentioned feeling respected and expressed respect for others within in the program. The shared leadership model implemented by the DHS Centers of Excellence programs may have been a reflection of the program structure.

\section{Subtheme 2: Program Structure}

\section{Finding 2. Programs focused on applied research.}

The second finding was that programs focused on applied research resulting in organizational structures segmented by discipline. Elements such as program structure, its implementation in a virtual environment, and its impact on participants were areas explored during this data-collection and analysis effort. Hoch and Kozlowski (2014) noted that shared leadership was related to team performance even in scenarios where the team was collocated. Hoch and Kozlowski focused on hierarchical leadership, structural supports, shared leadership, and their relationships to team performance. To conduct my analysis, I looked for evidence consistent with Wageman and Fisher's four areas of legitimate authority: (a) executing tasks, (b) monitoring and managing, (c) designing the team, and (d) guiding overall direction. My interpretation of the findings in my study indicated that multiple individuals conducted each of the four areas of legitimate authority. Executing tasks was most commonly associated with the graduate or postdoctoral researchers, whereas monitoring and managing were generally associated with technical area leads. Designing the team and a portion of guiding the overall direction fell to the top program leader(s). 
The structure of the programs described in the interviews was consistent across participants. Figure 21 depicts the structural features discussed by the participants. This structure consists of a top leader who was usually titled the director. The director represented a single academic organization and was responsible for providing the vision, distribution of funds, and selection of the technical of their program. At the technical-area level, a single organization was usually identified and offered a fair amount of autonomy to operate as a single team. Within a technical area, specific projects were distributed to individual researchers. These researchers may opt to conduct the research themselves or engage other researchers and assistants as needed. The individual researcher engaged the top-level organization to coordinate contractual agreements with partner researchers In the program structures described by the participants, there was autonomy at the researcher level to choose to coordinate versus collaborating. This flexibility resulted in varied responses from participants on their experiences regarding collaboration. Hoch and Kozlowski (2014) predicted the impact of structural behaviors based on reward systems. The structure of most of the examples of project work within a program resembled the silos discussed by Kuhn (2012) where academic departments behaved as competitors rather than collaborators. Although competition was not a common theme, the participants discussed the approach of segmenting the funding and work assignments. The segmented work was then coordinated to bring elements together for the final product. Future researchers may want to explore further the basis for separation of funds to determine if it negatively impacts overall collaborative efforts. 


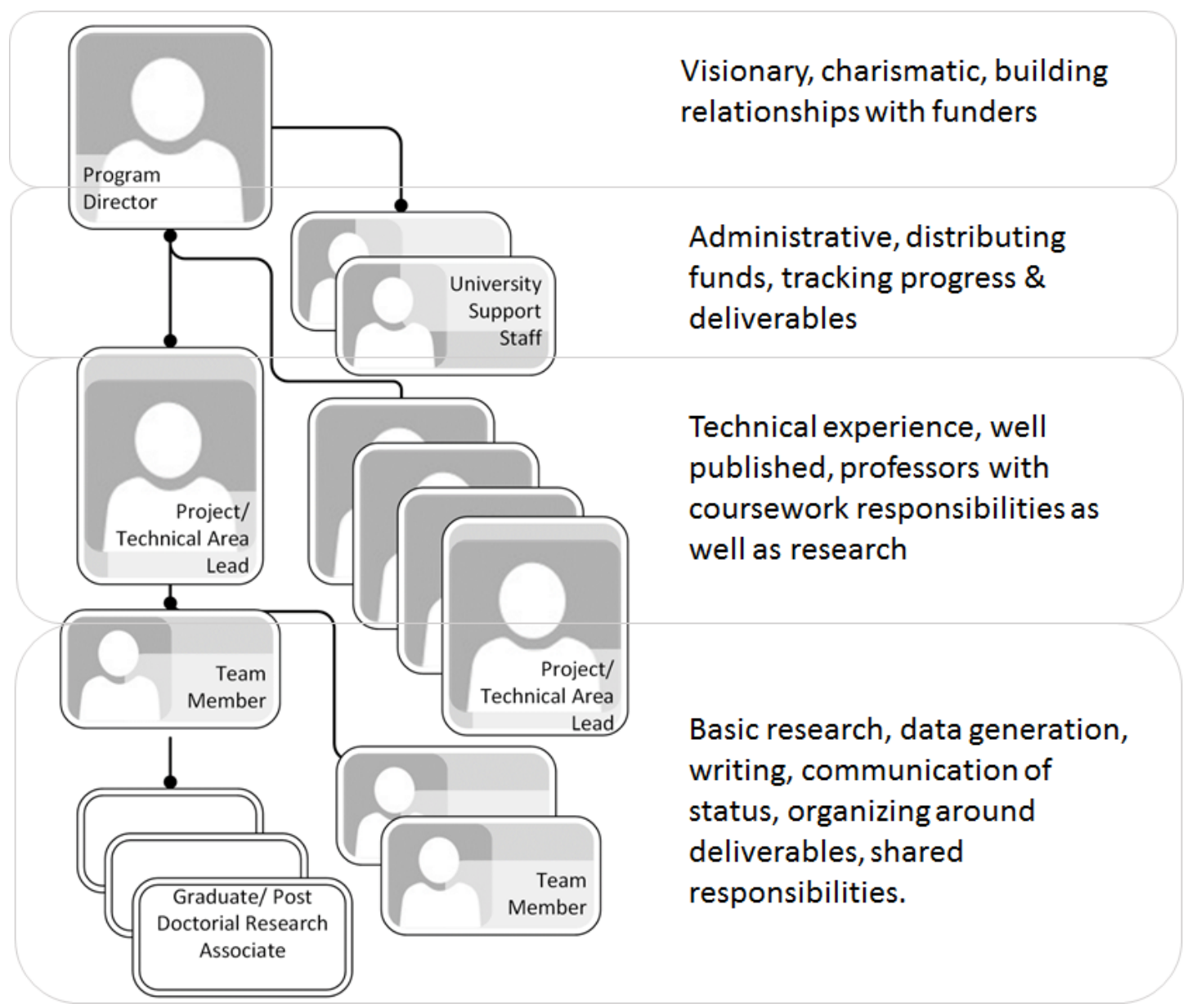

Figure 21. General program structure.

Program structure consisted of a top leader, who was usually noted as the

director. The programs were then subdivided into technical areas. Research teams were focused on project elements and included the graduate and postdoctoral research assistant participants.

In all participant interviews, the transactional leadership was located at the bottom of the structure. One participant discussed this subject at length. They noted that the early career researchers, as leaders, had the responsibility to set up meetings, pull information together, and conduct more in-depth research. They noted that the top, more charismatic, 
leadership of a program was very busy building relationships with sponsors and university administration. The technical area leaders were generally influential in their field and usually busy with university obligations, coursework, and oversite of postdoctoral researchers. The focused time and interest of early career researchers as transactional leaders made their contribution significant within these program structures. The amount of dependency on the early career members of the team may also contribute to the locally collaborative structure. One participated noted that the early career members of the team were less likely to reach out independently to the other organizations. This workload combined with the hierarchical structure may naturally limit direct collaboration outside of individual subteams and organizations.

Other drivers for collaborative behavior over coordinated behavior described by participants included a scenario where the researchers had moved organizations and were familiar with the previous institution's researchers' skills. In another scenario, the participant pointed out that it depended on the project. A small project could be done by a single researcher; however, a large or complex project required a larger, morecoordinated team and collaboration was focused at the inter-organizational project team level.

To understand how these program structures emerged, it was valuable to also look at the overall program drivers and purpose. Figure 22 provides a proposed research engagement framework I derived from the collection of interviews. Cross-walking outcomes with research engagement led to a matrix of possible research categories. 


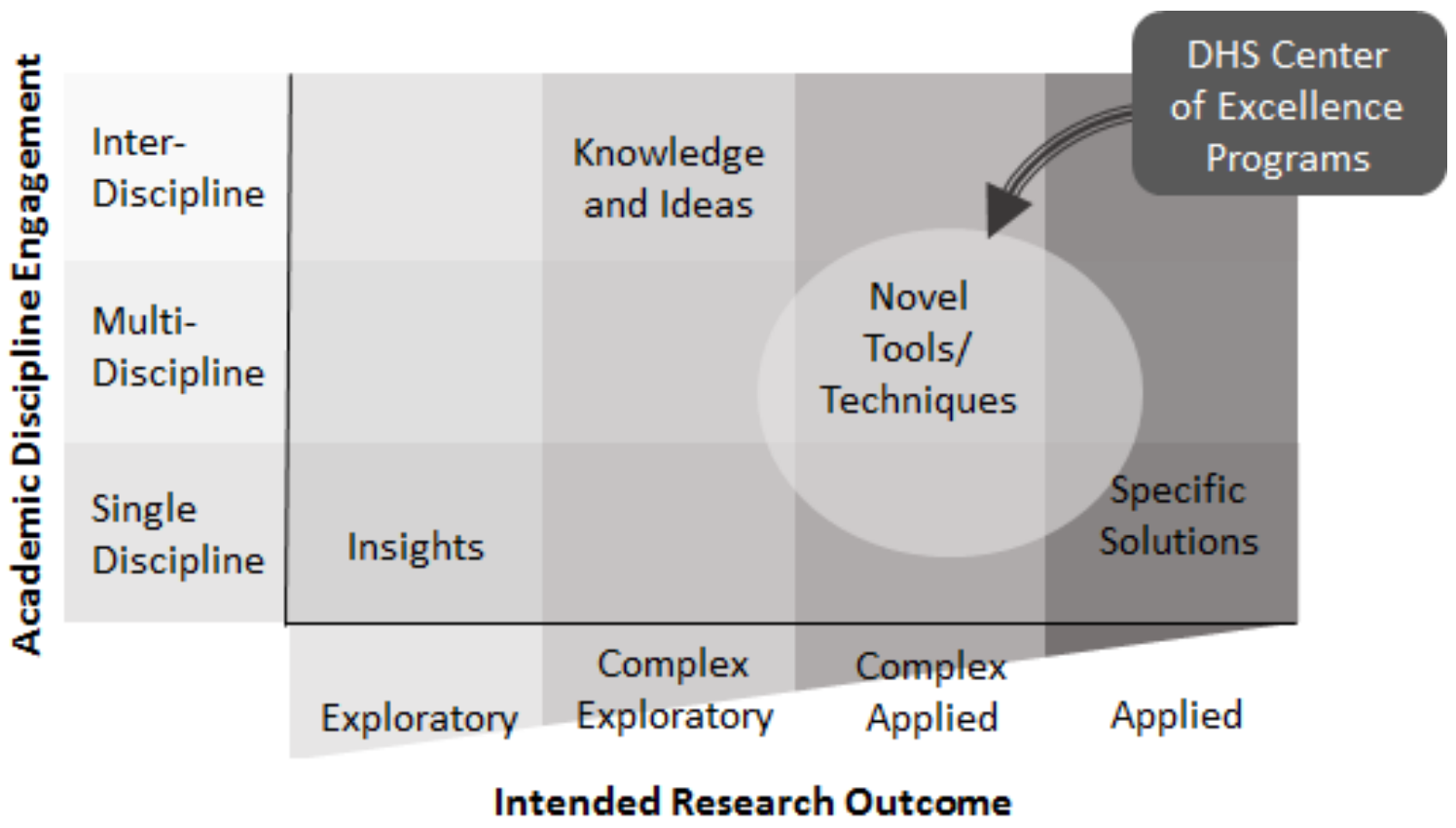

Figure 22. Proposed research engagement framework.

My engagement framework provides intended research outcomes cross-walked with academic discipline engagement options. Intended outcomes consist of a continuum from exploratory to applied. Engagement can be single, multiple, or interdisciplinary. Four intersections between outcome and engagement are included. A primarily applied and multidisciplinary juncture appears to be consistent with my findings for the DHS Centers of Excellence programs.

My proposed engagement framework provides a continuum of intended research outcomes ranging from basic exploratory to fully applied and defined. In the fundamental and fully exploratory extreme, outcomes were unknown. Along the continuum were basic research, which was more complex in nature, and exploratory research with undefined outcomes. Moving closer to fully applied was applied yet novel research. This stage of the research continuum included targeted outcomes with an eye toward the application. 
The applied extreme of the intended research outcome continuum was described by participants as applied and specifically defined. This kind of applied research was described as more of an engineering solution to a well-defined problem by the participants.

My proposed engagement framework provides the second dimension of research engagement focused on the academic discipline engagement. Academic discipline engagement includes three types of engagement. Single-discipline engagement, often a single researcher, was described by participants as work not coordinated with related research efforts of other disciplines. The multidisciplinary research was distinguished from interdisciplinary research by the level of coordination versus collaboration. In multidisciplinary teams, work was coordinated for a common outcome but did not require the integration of research to provide the expected outcome. In interdisciplinary research, more complex coordination was required bringing the specific information from different disciplines together for a unified result. The complex nature of these interdisciplinary collaborations was often exploratory in nature to allow for the researchers to integrate and adjust as needed throughout the research.

This research engagement framework provides a basis by which to understand why DHS Centers of Excellence programs conduct less integrative research. The programs' applied and coordinated nature at the top level appear to indicate a multidisciplinary structure dominated by coordination rather than collaboration activities. This engagement framework was consistent with findings by Bogers et al., (2017). The DHS Centers of Excellence programs appeared to be more aligned with extra- 
organizational, rather than inter-organizational, levels of analysis and research (Bogers et al., 2017). According to Bogers et al., this meant they had external stakeholders and functioned as individuals operating within a community. By contrast, inter-organizational analysis and research included alliances, the networked interaction between participants, and an ecosystem approach (Bogers et al., 2017). Bogers' description of an interorganizational program was consistent with the interdisciplinary element of my engagement framework.

The shared leadership and hierarchical organizational structure of DHS Centers of Excellence programs have direct implications for virtual leadership. Shared leadership described by participants resembled Hoch and Kozlowski's (2014) strategies for enabling shared leadership that required accepting the new paradigm of shared responsibility, teaming behaviors, respecting member competencies, and encouraging leadership behavior in others. Understanding the virtual environment related to these programs was important to establishing leader expectations.

\section{Subtheme 3: Virtual Environment}

\section{Finding 3. Coordination versus collaboration within programs.}

A notable finding in my research was the preference of coordination over collaboration behaviors between partners when considering the collocation versus virtual nature of the program participants. Collaboration occurred within collocated teams and coordination occurred between virtual partners. In the last 10 years, several important factors related to virtual environments have emerged, such as the type of work conducted virtually, trust, technology implementation, and redefinition of outcomes (Gilson et al., 
2014). Wadsworth and Blanchard (2015) looked at virtual team leadership as a process very different from face-to-face leadership. They examined influence tactics for both face-to-face and virtual leadership, assessing which was more successful. Wadsworth and Blanchard observed that virtual leaders need to spend more time in contact with team members. They highlighted technologies and characteristics of successful virtual leaders. In my research findings, the virtual nature of the programs was less noticeable. The frequent silo structure of the project teams within a program was often determined by proximity and common technical interests. The frequency of communication was generally weekly or monthly depending on the phase of the work. Again, early and late stages of project work were described as having more communication through leadership coordination.

Communication and trust-building were discussed in each participant interview, although not all participants used the word trust. All communication between individuals was not collocated and the information was often limited to phone calls and e-mails. One participant noted that e-mail and phone calls were fine when the message was positive, however, if the message was negative face-to-face meetings were preferred. Another participant noted that meeting held internal to the organization were often face-to-face in teams. Meetings with external collaborators were often held one-on-one.

Leadership responsibility for virtual coordination in these DHS Centers of Excellence programs may resemble market-based partnerships. Du et al. (2014) described two types of innovated projects, science-based and market-based. Their science-based model was characterized as inexpensive, lower risk, and a good source of specialized 
knowledge (Du et al., 2014). Market-based partnering was characterized as involving the customer, drawing on industry-specific expertise, and working as a community of practice (Du et al., 2014). Du et al. (2014) discussed the tradeoff between market-based and science-based innovative projects. Market-based projects required more structured management to ensure roles and responsibilities were clear and work could be coordinated and delivered. Science-based projects required less structure but had a higher risk of not producing a usable product. The formal management structure and customer involvement described by of DHS Centers of Excellence program participants in my study appear to be more closely aligned with the market-based partnering. Participants discussed the engagement of actual users and the challenges to collaboration given the specific outcomes associated with individual DHS Centers of Excellence programs. The expectation of implementation of outcomes was consistent across all participants. One participant noted the success of the program was correlated to the fact that they worked closely with the users and produced a tangible product.

It was interesting, then, to consider this market-based perspective in conjunction with the research culture. The concept of how leadership, structure, and environmental impact motivation of research participants was of specific interest for my research. Understanding more about research culture was helpful in learning more about motivation.

\section{Subtheme 4: Research Culture}

Finding 4. Program members were primarily self-motivated. 
The forth finding was that program members were primarily self-motivated with publishing serving as evidence of respected behavior. For my study, scientific research and development behavior was collectively discussed as a research culture. Specifically, this research culture stems from having a research environment within an academic setting. Shrum et al. (2007) included this culture as important to consider. Kuhn (2012) discussed the competition for funding and prestige. The participants in this research touched on all of these aspects. As I noted above, external collaboration at the project level was often a result of previous research engagements with relationships built over time. This previous experience with a collaborator resulted in increased knowledge of another researcher's skillset and an increased level of respect. Erdogan and Bauer (2014) provided a basis for leader-member exchange quality in the relationship, including effect, loyalty, and professional respect. It was possible respect and trust were used synonymously within this research culture, as trust was a key component of previous research on collaboration (McNab et al., 2012).

Walsh and Huang (2014) researched collaborating scientists from the U.S. and Japan facing the challenges of collaborating in a competitive scientific environment. To understand the drivers and barriers to scientific collaboration, previous researchers have also explored patents and copatents (Belderbos et al., 2014). One metric relating to Belderbos et al., (2014) was the incentive to publish. Publishing was a measurable way to produce and share scientific knowledge. Two of the participants were passionate about the opportunity to publish. One participant noted the opportunity to publish as the basis for early career members of the team to take ownership and exert leadership. The other 
expressed the scientific responsibility of sharing your findings. Scientific collaborations of any kind were considered advantageous to researchers when viewed from the publication perspective (Lariviere et al., 2015). In my research, publication collaboration also resembled coordination of information, rather than full collaborative development, with various partners developing sections or chapters of the final research products.

Specific to organizational incentives, Lee et al. (2015) found that organizational performance appraisal systems needed to match the expected attitude of the follower to engage the leader as expected. The organizational incentives discussed by the participants indicated that incentives were targeted at keeping funding within the single organization whenever possible. Coordination was encouraged, but collaboration was often limited to within the same institution. Jay (2013) noted that these hybrid organizations required new organizational success criteria. The participant's descriptions of the structured and coordinated management of DHS Centers of Excellence programs did not provide evidence of novel or unique organizational incentives at play in these programs.

Another common theme was the role of scientific prestige (Walsh \& Huang, 2014). It appeared the reputation of the scientists involved played a key role in the coordination and collaboration. Scientific prestige and publications were noted as helping to make connections and add strength to a proposal. One participant noted that the most intense coordination occurred during the proposal phase of the work. Research coordination and reputation for delivery were heavily depended upon when deliverables were due. Reputation was perceived to be a reflection of the reliability to deliver as agreed. 
Key findings in my research included evidence of shared leadership implemented by DHS Centers of Excellence programs implemented shared leadership. These programs focused on applied research resulting in organizational structures segmented by discipline. Collaboration occurred within collocated teams with coordination occurring between virtual partners. Program members were primarily self-motivated with publishing serving as evidence of respected behavior.

\section{Limitations of the Study}

Limitations to my study stemmed from three aspects. First, the self-selection of participants introduced a possible limitation as a result of response bias in data collection. Second, the narrow focus of my population on the DHS Centers of Excellence programs as a target program set could be limiting. Third, there is limited extensibility of my study based on the qualitative approach I implemented focusing on gaining additional understanding.

My study reached out to more than 700 individual participants from 17 DHS Centers of Excellence programs stood up before 2017. Fifteen of those individuals, representing 10 of the Centers of Excellence programs, returned the initial request indicating an agreement to be considered for this research. The nature of the process of sending e-mails and allowing participants to self-select introduces a possible response bias to my study. For the 16 individuals agreeing to participate initially, eight were nonresponsive to the consent request for an interview sent both in the summer and the fall. To ensure this subset of potential participants, who had already expressed a willingness to participate, had the full opportunity to participate, I sent an additional 
follow-up request in the second half of the school year (second semester). Seven of the remaining eight potential participants responded positively to this third request. Future researchers interested in the subject of multiorganizational research and development programs involving universities may also want to take into account the academic calendar and timing of researcher availability. My study's offers only an initial insight into the virtual leadership of multiorganizational scientific collaboration programs. The findings from this study provided the theoretical basis and empirical evidence for more expansive and quantitative research projects.

The information gathered was specific to DHS programs (U.S. DHS, 2017). Their programs were specifically defined as being run by universities. It was possible this population was not wholly representative of other scientific collaboration programs. Future researchers may want to take a cross-section of programs from a variety of departments within the government (U.S. DOE, 2015).

I selected a qualitative approach for this study focusing on gaining additional understanding. The consistency in the findings could inform additional research and potential surveys, focus groups, or other quantitative methods. The findings require additional research through statistically defensible approaches to gain general insights applicable to broader populations.

\section{Recommendations}

A surprising finding of my research was the program segmentation into what resembles Kuhn's (2012) silos. This was a surprising finding, given the expressed multiorganizational collaboration expectation by the U.S. Congress in requesting the 
implementation of the DHS Centers of Excellence programs. Future researchers may want to expand my proposed research engagement framework as it might apply to university incentives. Using this framework may provide opportunities to create novel organizational incentives enhancing the outcomes of these multiorganizational programs.

Initial evidence showed agile-management philosophies might be consistent with the shared leadership model for program management (Highsmith, 2001; Denning, 2013; Ramos et al., 2013; Hilt et al., 2016). A future research area might include a specific analysis of agile-management processes as they might be applied to multiorganizational research and development programs. This analysis could highlight opportunities to enhance the management tools and methods currently used in these programs.

It was possible respect and trust were used synonymously within this academic environment, as trust was a key component of previous research on collaboration. McNab et al. (2012) included an evaluation of trust and relationship building. The concept of respect in research culture and academic environment could be an indication of trust. Future research could be focused on respect and whether or not it was an adequate representation of trust. Research conducted to compare and contrast the concepts of respect and trust would enhance understanding of the applicability of my study.

The consistency in the findings and my proposed research engagement framework could inform additional research and potential surveys, focus groups, or other quantitative research exploring complex multiorganizational research and development programs. The extensibility of my study could be improved with additional research through statistically defensible approaches to gain general insights applicable to broader 
populations. The information gathered here was specific to DHS Centers of Excellence programs (U.S. DHS, 2017). Their programs were specifically defined as being run by universities. It was possible my population was not wholly representative of other complex scientific collaboration programs. Future researchers could take a cross-section of programs from a variety of departments within the government (U.S. DOE, 2015) to understand if the findings presented here were more broadly applicable.

\section{Implications}

My study illuminated the intersection of institutionally complex programs and the influence of highly educated participant research on the resulting programs operating in a multiorganizational environment. This environment presented a new area of organizational leadership research. My study focused specifically on the exploration of virtual leadership strategies within DHS Centers of Excellence programs. The multilayer interactions with collaboration occurred within collocated teams and coordination occurred between virtual partners, adding complexity warranting additional exploration. The results provided here represent member-based insights on leadership strategies, which could support the development of virtual leadership training in preparation for assembly of future multiorganizational programs. Given the key role of graduate and postdoctoral researchers within these programs, targeted coursework on leadership for graduate students could fit nicely into the overall program expectations. Agilemanagement practices might be a starting point for this training.

Actionable information and insights provided by my research engagement framework provided to program owners may result in enhanced leadership effectiveness 
and selection of multiorganizational program management. Over the longer term, this study could be the basis for enhanced leadership education for researchers.

Investigation of the leadership of multiorganizational research and development programs could influence positive social change in practice through vetting the research engagement framework proposed here to inform future leadership models. My study diverged from traditional leadership research which commonly addressed leadership traits related to organizational outcomes. Instead, my research brought together discipline engagement and outcomes from the participants' perspectives. My study adds to organizational research by including evidence and observations drawn from interviews from participants in an academic environment. My observations expressing alternative views of organizational leadership research. Insights can be gained by understanding coordination and collaboration as program owners envisioned it in contrast program participants described it.

My study on multiorganizational research and development programs was in response to a call from past researchers to address the challenges of understanding complex systems leadership from a follower's perspective. Anand et al. (2011) called for additional learning on complex systems and leader-member exchange theory. Bligh (2011) called for specific follower-centered research. The exploration of multiorganizational research and development program leadership from the members' perspective affects positive social change by capturing and sharing the voice of the affected participants. Follower viewpoints provide unique insight and perspective to additional targets of social change. Targeted research to further my findings would lead 
to positive social change through moving to a more transformational model of scientific collaboration within these programs, starting with improved training for future leaders of similar complex multiorganizational virtual programs. Considering the perspective of highly educated participants in complex multiorganizational teams contributes to current organizational and leadership theory. These research results are expected to inform future studies on leadership strategies and the influences of research culture.

The investigation into the complex leadership of multiorganizational research and development programs may also contribute to a positive social change in extending extant management knowledge. Targeted training consistent with these findings would lead to positive social change through moving to a more transformational model of scientific collaboration within these programs, starting with improved training for future leaders of similar complex multiorganizational virtual program. Potential modifications in training focused on management for scientific leaders would better prepare them for complex organizational leadership environments requiring coordination of virtual partners. Improved training could lead to a larger pool of leadership resources for multiorganizational collaborations, which could have national and global impacts through increased innovation.

\section{Conclusions}

After receiving large monetary investments in science innovation, it was imperative that leaders of these complex scientific collaboration programs recognize the need to fully understand the leadership paradigm and explore areas for improvement. Scientists assuming leadership roles often practice collaborations on a small scale within 
a single discipline. However, they may lack experience, training, or incentives to extend this knowledge to the virtual leadership of complex multiorganizational programs. Leader-member exchange and complex systems theories served as a conceptual framework to explore leadership strategies with the distinction between coordinated research and collaborative research. Virtual leadership was reserved primarily for coordination between organizations. I used a qualitative case study represented by the collection of U.S. DHS's Centers of Excellence programs. Thirteen individuals were interviewed, representing 10 of these programs. Four findings were identified when asking: what virtual leadership strategies do leaders of multiorganizational collaboration programs use to motivate their highly educated scientists across organizations? In finding 1, within the leadership subtheme, I elucidated that programs implemented a sharedleadership model with transformational and transactional leadership occurring at all levels. Collectively finding 2 , within the program structure subtheme, I demonstrated that programs focused on applied research resulting in organizational structures segmented by discipline. Within finding 3, aligned with the virtual environment subtheme, I showed collaboration occurred within collocated teams and coordination occurred between virtual partners. In finding 4, within the research culture subtheme, I found program members were primarily self-motivated with publishing serving as evidence of respected behavior. Targeted training consistent with these findings would lead to positive social change through moving to a more transformational model of scientific collaboration within these programs, starting with improved training for future leaders of similar complex multiorganizational virtual program. 
Across all interviews conducted for my research, participants were self-motivated and excited about the opportunity to develop new ideas, provide impact, and produce publications. These characteristics were consistent with an academic environment and may be unique to research programs led by universities. Permanent technical staff served as project leads, and the early career members of the project teams conducted much of the research itself as well as leadership activities for daily tasking. Early career members of research teams played a critical transactional leadership role in conducting and communicating research. Korman (1966) described these type of leadership activity as initiating structure. Bass (1997) later drew in the initiating structure behaviors into the description of transactional leadership concepts. More recently, leadership researcher's have drawn on these early concepts to understand the relationship between leaders and their team members when considering the member perspective. (Gaudet \& Tremblay, 2017). Transactional leadership was a complementary concept to transformational leadership (Bass 1997).

A surprising finding of my research was the program segmentation into what resembles Kuhn's (2012) silos. This was a surprising finding, given the expressed multiorganizational collaboration expectation by the U.S. Congress in requesting the implementation of scientific collaborations such as the DHS Centers of Excellence programs. Multiple universities were brought together for these programs. The funding and work were typically broken down by the organization. The research was generally conducted within, rather than between, organizations. Researchers were also frequently collocated with their project teams. Collaboration primarily occurred within collocated 
teams. Coordination was identified as the most common interaction across organizations virtual partners. A research engagement framework was provided as a starting point for understanding and investigating the complex program structures within research programs. Future researchers may want to expand my engagement framework as it might extend to university incentives. Considering the implications of my findings may provide a starting point for looking for opportunities it creates novel or unique organizational incentives that may enhance the outcomes of these multiorganizational programs. 


\section{References}

ADAC (2017). Arctic domain awareness center of excellence. Retrieved from https://www.hsuniversityprograms.org/centers/adac-arctic-domain-awareness/

Ale Ebrahim, N. (2015). Virtual R\&D teams: A new model for product development. International Journal of Innovation-IJI, 3(2), 01-27. doi:10.5585/iji.v3i2.43

ALERT (2017). Center of excellence for awareness \& localization of explosives-related threats. Retrieved from https://www.hsuniversityprograms.org/centers/alertexplosives-detection/

Anand, S., Hu, J., Liden, R. C., \& Vidyarthi, P. R. (2011) Leader-member exchange: Recent findings and prospects for the future. In A. Bryman, D. L. Collinson, K. Grint, B. Jackson, \& M. Uhl-Bien (Eds.), The Sage handbook of leadership (pp. 311-345). Thousand Oaks, CA: Sage.

Avolio, B. J., Sosik, J. J. Kahai, S. S., \& Baker, B. (2014) E-leadership: Re-examining transformations in leadership source and transmission. The Leadership Quarterly, 25, 105-131. doi:10.1016/j.leaqua.2013.11.003

Bass, B. M. (1997). Does the transactional-transformational leadership paradigm transcend organizational and national boundaries? American Psychologist, 52(2), 130-139. Retrieved from http://www.communicationcache.com/uploads/1/0/8/8/10887248/does_the_transa ctionaltransformational_leadership_paradigm_transcend_organizational_and_nati onal_boundaries.pdf

Bedwell, W. L., Wildman, J. L., DiazGranados, D., Salazar, M., Kramer, W. S., Salas, E. 
(2012). Collaboration at work: An integrative multilevel conceptualization. Human Resource Management Review, 22, 128-145. doi:10.1016/j.hrmr.2011.11.007

Belderbos, R., Cassiman, B., Faems, D., Leten, B., \& Van Looy, B. (2014). Coownership of intellectual property: Exploring the value-appropriation and valuecreation implications of co-patenting with different partners. Research Policy, 43, 841-852. doi:10.1016/j.respol.2013.08.013

Berry, P. (2013). The impact of globalization and technology on teaching business communication: Reframing and enlarging worldview, methods, and content. American Journal of Business Education, 6, 57-66. doi:10.19030/ajbe.v6i1.7483

Biesenthal, C., \& Wilden, R. (2014). Multi-level project governance: Trends and opportunities. International Journal of Project Management, 32, 1291-1308. doi:10.1016/j.ijproman.2014.06.005

Bligh, M. C. (2011). Followership and follower-centered approaches. In A. Bryman, D. L. Colinson, K. Grint, B. Jackson, \& M. Uhl-Bien (Eds.), The Sage handbook of leadership (pp. 425-436). Thousand Oaks, CA: Sage.

Bogers, M., Zobel, A. K., Afuah, A., Almirall, E., Brunswicker, S., Dahlander, L., . . \& Ter Wal, A. L. J. (2017). The open innovation research landscape: Established perspectives and emerging themes across different levels of analysis. Industry and Innovation, 24(1), 8-40. doi:10.1080/13662716.2016.1240068

Bosch-Sijtsema, P. M., Fruchter, R., Vartianinen, M., \& Ruohomaki, V. (2011). A framework to analyze knowledge work in distributed teams. Group \& 
Organization Management, 36, 275-307. doi:10.1177/1059601111403625

Bozeman, B., Fay, D., \& Slade, C. P. (2013). Research collaboration with universities and academic entrepreneurship: The-state-of-the-art. The Journal of Technology Transfer, 38, 1-67. doi:10.1007/s10961-012-9281-8

BTI (2017). Borders, trade, and immigration institute. Retrieved from https://www.hsuniversityprograms.org/centers/bti-border-security/

Cady, P. (2016). A system of system lenses for leadership decision-making. Healthcare Management Forum, 29, 8-11. doi:10.1177/0840470415614412

CAMRA (2017). Center for advancing microbial risk assessment. Retrieved from https://www.hsuniversityprograms.org/centers/former/camra/

CAOE (2017). Center for accelerating operational efficiency. Retrieved from https://www.hsuniversityprograms.org/centers/center-for-acceleratingoperational-efficiency/

Carter, D. R., \& DeChurch, L. A. (2014). Leadership in multiteam systems: A network perspective. In V. D. Day (Ed.), The Oxford handbook of leadership and organizations (pp. 482-502). New York, NY: Oxford University Press. CHC (2017). Coastal hazards center of excellence. Retrieved from http://coastalhazardscenter.org/

Cho, A. (2013, May 24). Hubs aim to reinvent DOE research culture. Science, 340, 914 918. doi:10.1126/science.340.6135.914

Choy, J., McCormack, D., \& Djurkovic, N. (2016), Leader-member exchange and job performance. Journal of Management Development, 35, 104-119, 
doi:10.1108/JMD-06-2015-0086

Chompalov, I. (2014). Lessons learned from the study of multi-organizational collaborations in science and implications for the role of the university in the 21 century. In H. Marcel (Ed.), The institution of science and the science of institutions: The legacy of Joseph Ben-David (pp. 167-184). doi:10.1007/978-94007-7407-0_9

CINA (2017). Criminal investigations and network analysis. Retrieved from https://www.hsuniversityprograms.org/centers/

CIRI (2017). Critical infrastructure resilience institute. Retrieved from http://ciri.illinois.edu/about

Cole, M. S., Bedeian, A. G., \& Bruch, H. (2011). Linking leader behavior and leadership consensus to team performance: Integrating direct consensus and dispersion models of group composition. The Leadership Quarterly, 22, 383-398. doi:10.1016/j.leaqua.2011.02.012

Collinson, D. (2014). Dichotomies, dialectics, and dilemmas: New directions for critical leadership studies? Leadership, 10, 36-55. doi:10.1177/1742715013510807

CRC (2017). Coastal resilience center of excellence. Retrieved from https://www.hsuniversityprograms.org/centers/crc-coastal-resilience/

CREATE (2017). National center for risk and economic analysis of terrorism events. Retrieved from https://www.hsuniversityprograms.org/centers/emeritus/createrisk-economic-analysis/

CVADA (2017). Center for visualization and data analytics. Retrieved from 
https://www.hsuniversityprograms.org/centers/emeritus/cvada-data-visualanalytics/

Daim, T. U., Ha, A., Reutiman, S., Hughes B., Pathak, U., Bynum, W., \& Bhatla, A. (2012). Exploring the communication breakdown in global virtual teams. International Journal of Project Management, 30, 199-212. doi:10.1016/j.ijproman.2011.06.004

Day, D. V. (2014a). The future of leadership: Challenges and prospects. In V. D. Day (Ed.), The Oxford handbook of leadership and organizations (pp. 859-867). New York, NY: Oxford University Press.

Day, D. V. (2014b). Leadership and organizations. In V. D. Day (Ed.), The Oxford handbook of leadership and organizations (pp. 3-12). New York, NY: Oxford University Press.

Day, D. V., Griffin, M. A., \& Louw, K. R. (2014). The climate and culture of leadership in organizations. In B. Schneider \& K. M. Barbera (Eds.), The Oxford handbook of organizational climate and culture (pp. 101-117). New York, NY: Oxford University Press.

Denning, S. (2010). The leader's guide to radical management. San Francisco, CA: Josey-Bass.

Denning, S. (2012a). The reinvention of management. Strategy \& Leadership, 39(2) 917. doi: $10.1108 / 10878571111114428$

Denning, S. (2012b). How agile can transform manufacturing: The case of Wikispeed. Strategy \& Leadership, 40(6), 22-28. doi:10.1108/10878571211278859 
Denning, S. (2013). Why agile can be a game changer for managing continuous innovation in many industries. Strategy \& Leadership, 41(2), 5-11. doi:3A10.1108/2F10878571311318187

Diaz-Saenz, H. R. (2011) Transformational leadership. In A. Bryman, D. L. Colinson, K. Grint, B. Jackson, \& M. Uhl-Bien (Eds.), The Sage handbook of leadership (pp. 199-310). Thousand Oaks, CA: Sage.

Dinh, J. E., Lord, R. G., Gardner, W. L., Meuser, J. D., Liden, R. C., \& Hu, J. (2014). Leadership theory and research in the new millennium: Current theoretical trends and changing perspectives. The Leadership Quarterly, 25(1), 36-62. doi:10.1016/j.leaqua.2013.11.005

Du, J., Leten, B., \& Vanhaverbeke, W. (2014). Managing open innovation projects with science-based and market-based partners. Research Policy, 43(5), 828-840. doi:10.1016/j.respol.2013.12.008

Dyer, W. G. Jr., Dyer, J. H., \& Dyer, W. G. (2013). Team building: Proven strategies for improving team performance. ( $5^{\text {th }}$ ed.). CA: Jossey-Bass

Edmonstone, J. (2016). Complex adaptive leadership: embracing paradox and uncertainty. Action Learning: Research and Practice, 1-3. doi:10.1080/14767333.2015.1130353

Elfner, L. E., Falk-Krzenski, H. J., Sullivan, K., Velkey, A., Illman, D. L., Baker, J., \& Pita-Szczesniewki, A. (2011). Team science: Heaving walls and melding silos [Sigma Xi white paper]. Retrieved from http://www.sigmaxi.org/programs/issues/TeamScience.pdf 
Ellen, B. P. (2014). Considering the positive possibilities of leader political behavior. Journal of Organizational Behavior, 35, 892-896. doi:10.1002/job.1938

Emery, F. E., \& Trist, E. L. (1965). The causal texture of organizational environments. In W. W. Burke, D. G. Lake, \& J. L. Paine (Eds.), Organization change: A comprehensive reader (pp. 7-20). San Francisco, CA: Jossey-Bass.

Erdogan, B., \& Bauer, T. N. (2014) Leader-member exchange (LMX) theory: The relational approach to leadership. In V. D. Day (Ed.), The Oxford handbook of leadership and organizations (pp. 407-433). New York, NY: Oxford University Press.

Erkens, M., Bodemer, D., \& Hoppe, H. U. (2016). Improving collaborative learning in the classroom: Text mining based grouping and representing. International Journal of Computer-Supported Collaborative Learning, 11(4). 387-415. doi:10.1007/s11412-016-9243-5

Fairhurst, G. T., \& Uhl-Bien, M. (2012) Organizational discourse analysis (ODA): Examining leadership as a relational process. Leadership Quarterly, 23, 10431062. doi:10.1016/j.leaqua.2012.10.005

Felix, C., Pandey, A. V., \& Bertini, E. (2017). TextTile: An interactive visualization tool for seamless exploratory analysis of structured data and unstructured text. IEEE Transactions on Visualization and Computer Graphics, 23(1), 161-170. doi:10.1109/TVCG.2016.2598447

FPDI (2017). Food protection and defense institute. Retrieved from https://www.hsuniversityprograms.org/centers/emeritus/fpdi-food-defense/ 
Freeman, M., Baurmann, A., Fisher, A., Blythe, J., \& Akhtar-Danesh, N. (2012). Case study methodology in nurse migration research: An integrative review. Applied Nursing Research, 25, 222-228. doi:10.1016/j.apnr.2012.02.001

Fusch, P. I., \& Ness, L. R. (2015). Are we there yet? Data saturation in qualitative research. The Qualitative Report, 20(9), 1408-1416. Retrieved from http://nsuworks.nova.edu/tqr/vol20/iss9/3/

Garrison, G., Wakefield, R. L., Xu, X., \& Kim, S. H. (2010). Globally distributed teams: The effect of diversity on trust, cohesion and individual performance. Association for Computer Machinery Newsletter, 41(3), 27-48. doi:10.1145/1851175.1851178

Gaudet, M. C., \& Tremblay, M. (2017). Initiating structure leadership and employee behaviors: The role of perceived organizational support, affective commitment and leader-member exchange. European Management Journal, 35(5), 663-675. doi:10.1016/j.emj.2017.04.001

Gerstein, D. M. (September 18, 2015) Making DHS more efficient: Industry recommendations to improve homeland security. Testimony submitted to the House Homeland Security Committee, Subcommittee on Oversight and Management Efficiency, The RAND Corporation. Retrieved from http://tinyurl.com/gpqmag8

Gilson, L. L., Maynard, M. T., Young, N. C. J., Vartiainen, M., \& Hakonen, M. (2015). Virtual teams research 10 years, 10 themes, and 10 opportunities. Journal of Management, 41(5), 1313-1337. doi:10.1177/0149206314559946 
Halal, W. E. (2013). Forecasting the technology revolution: Results and learnings from the TechCast project. Technological Forecasting and Social Change, 80, 16351643. doi:10.1016/j.techfore.2013.02.008.

Hannah, S. T., Avolio, B. J., Walumbwa, F. O., \& Chan, A. (2012). Leader self and means efficacy: A multi-component approach. Organizational Behavior and Human Decision Processes, 118, 143-161. doi:10.1016/j.obhdp.2012.03.007

Hanssen, G. K. (2012). A longitudinal case study of an emerging software ecosystem: Implications for practice and theory. The Journal of Systems and Software, 85, 1455-1466. doi:10.1016/j.jss.2011.04.020

Havre, S., Hetzler, B., \& Nowell, L. (2000). ThemeRiver: Visualizing theme changes over time. Information Visualization, 115-123. doi:10.1109/INFVIS.2000.885098

Hazy, J. K., \& Uhl-Bien, M. (2015). Towards operationalizing complexity leadership: How generative, administrative and community-building leadership practices enact organizational outcomes, Leadership, 11(1), 79-104. doi:10.1177/1742715013511483

Hennink, M. M., Kaiser, B. N., \& Marconi, V. C. (2017). Code saturation versus meaning saturation: How many interviews are enough? Qualitative health research, 27(4), 591-608. doi:10.1177/1049732316665344

Henry, E. L. (2015). Facilitative boundary leadership: Enabling collaboration in complex, multi-organizational work (Unpublished doctoral dissertation). Harvard University.Retrieved from http://nrs.harvard.edu/urn-3:HUL.InstRepos:17464431

Hilt, M. J., Wagner, D., Osterlehner, V., \& Kampker, A. (2016) Agile predevelopment of 
production technologies for electric energy storage systems: A case study in the automotive industry, Procedia CIRP 50, 88-93. doi:10.1016/j.procir.2016.04.189

Hoch, J. E., \& Kozlowski, S. W. (2014). Leading virtual teams: Hierarchical leadership, structural supports, and shared team leadership. Journal of applied psychology, 99(3), 390-403. doi:10.1037/a0030264

Hoegl, M., \& Muethel, M. (2016). Enabling Shared Leadership in Virtual Project Teams: A Practitioners' Guide. Project Management Journal, 47, 7-12. doi:10.1002/pmj.21564

Homeland Security Act of 2002, Pub. L. 107-296, 6 U.S.C. $188 \S 308$

Hudson, D. L. (2013). Attachment theory and leader-follower relationships. The Psychologist-Manager Journal, 16(3), 147-159. doi:10.1037/mgr0000003

Huettermann, H., Doering, S., \& Boerner, S. (2014). Leadership and team identification: Exploring the followers' perspective. The Leadership Quarterly, 25, 413-432. doi:10.1016/j.leaqua.2013.10.010

Jay, J. (2013). Navigating paradox as a mechanism of change and innovation in hybrid organizations. Academy of Management Journal, 56, 137-159. doi:10.5465/amj.2010.0772

Jirotka, M., Lee, C. P., \& Olson, G. M. (2013). Supporting scientific collaboration: Methods, tools, and concepts. Computer Supported Cooperative Work, 22, 667715. doi:10.1007/s10606-012-9184-0

Korman, A. K. (1966). “Consideration," "Initiating Structure,” and Organizational Criteria-a Review. Personnel Psychology, 19(4), 349-361. doi:10.1111/j.1744- 
6570.1966.tb00310.x

Krallinger, M., Rabal, O., Lourenço, A., Oyarzabal, J., \& Valencia, A. (2017).

Information retrieval and text mining technologies for chemistry. Chemical Reviews. doi:10.1021/acs.chemrev.6b00851

Kuhn, T. S. (2012). The structure of scientific revolutions (3rd ed.). Chicago: University of Chicago Press.

Kwon, H., Kim, J., \& Park, Y. (2017). Applying LSA text mining technique in envisioning social impacts of emerging technologies: The case of drone technology. Technovation, 60, 15-28. doi.org/10.1016/j.technovation.2017.01.001

Lalor, J. G., Casey, D., Elliott, N., Coyne, I., Comiskey, C., Higgins, A., . . \& \& Begley, C. (2013). Using case study within a sequential explanatory design to evaluate the impact of specialist and advanced practice roles on clinical outcomes: The SCAPE study. BMC Medical Research Methodology, 13, 55. doi:10.1186/14712288-13-55

Lariviere, V., Gingras, Y., Sugimoto, C. R., \& Tsou, A. (2015). Team size matters: Collaboration and scientific impact since 1900. Journal of the Association for Information Science and Technology, 66, 1323-1332. doi:10.1002/asi.23266

Lee, A., Martin, R., Thomas, G., Guillaume, Y., \& Maio, G. R. (2015). Conceptualizing leadership perceptions as attitudes: Using attitude theory to further understand the leadership process. The Leadership Quarterly 26, 910-934. doi:10.1016/j.leaqua.2015.10.003

Leiserson C. E., \& McVinney, C. (2015, July 16). Science professors need leadership 
training. Nature, 523, 279-281. Retrieved from

https://www.nature.com/polopoly_fs/1.17955!/menu/main/topColumns/topLeftCo lumn/pdf/523279a.pdf

Long, J. C., Cunningham, R. C., Wiley, J. Carswell, P., \& Braithwaite, J. (2013). Leadership in complex networks: The importance of network position and strategic action in a translational cancer research network. Implementation Science, 8, 122. doi:10.1186/1748-5908-8-122

Luciano, M. M., DeChurch, L. A., \& Mathieu, J. E. (2015). Multiteam systems: A structural framework and meso-theory of system functioning. Journal of Management. Advance online publication. doi:10.1177/0149206315601184

Mainemelis, C., Kark, R., \& Epitropaki, O. (2015). Creative leadership: A multi-context conceptualization. The Academy of Management Annals, 9, 393-482. doi:10.1080/19416520.2015.1024502

Malterud, K., Siersma, V. D., \& Guassora, A. D. (2016). Sample size in qualitative interview studies: guided by information power. Qualitative health research, 26(13), 1753-1760. doi:10.1177/1049732315617444

Manning, S., \& Roessler, D. (2014). The formation of cross-sector development partnerships: How bridging agents shape project agendas and longer-term alliances. Journal of Business Ethics, 123, 527-547. doi:10.1007/s10551-013$1853-5$

Manusov, E., Ronnau, J., Vela, L., Lyndia, A., \& Galke, C. (2015). Engagement and coproduction: Building an inter-professional integrated medical education and 
clinical practice. International Journal of Integrated Care, 15(5). doi:10.5334/ijic. 2105

Marion, R., \& Uhl-Bien, M. (2011), Implications of complexity science for the study of leadership.In P. Allend, S. Maguire, \& B. McKelvey (Eds.), The Sage handbook of complexity and management (pp. 385-399). Thousand Oaks, CA:Sage.

Mars, M. M., Bronstein, J. L., \& Lusch, R. F. (2012). The value of a metaphor: Organizations and ecosystems. Organizational Dynamics, 41, 271-280. doi:10.1016/j.orgdyn.2012.08.002

Mason, M. (2010). Sample size and saturation in Ph.D. studies using qualitative interviews. Forum Qualitative Sozialforschung / Forum: Qualitative Social Research, 11(3). doi:10.17169/fqs-11.3.1428.

Matzler, K., Strobl, A., \& Bailom, F. (2016). Leadership and the wisdom of crowds: how to tap into the collective intelligence of an organization. Strategy \& Leadership, 44(1), 30-35. doi:10.1108/SL-06-2015-0049

Maxwell, J. (2013). Qualitative research design: An interactive approach (3rd ed.). Thousand Oaks, CA: Sage.

McFarlane, D. A., \& Cooper, T. V. (2014). Brief synthesis of team leadership effectiveness and performance. Management and Administrative Sciences Review, 3, 221-225.

McNab, A. L., Basoglu, K. A., Sarker, S., \& Yu, Y. (2012). Evolution of cognitive trust in distributed software development teams: A punctuated equilibrium model. Electronic Markets, 22, 21-36. doi:10.1007/s12525-011-0081-z 
Merriam, S. B. (1998). Qualitative research and case study applications in education. San Francisco, CA: Jossey-Bass.

Merriam, S. B. (2009). Qualitative research: A guide to design and implementation. San Francisco, CA: Wiley.

Merriam, S. B., \& Tisdell, E. J. (2016) Qualitative research: A guide to design and implementation. San Francisco, CA: Wiley.

Microsoft. (2015). Lync 2013[Computer software]. Retrieved from http://products.office. com/en-us/Lync/lync-2013-video-conferencing-meeting-software.

Miles M. B., Huberman, \& Saldana J. (2014). Qualitative data analysis: A methods sourcebook ( $3^{\text {rd }}$ ed.). Thousand Oaks, CA: Sage.

MIREES (2017). Center for maritime, island and remote and extreme environment security. Retrieved from https://www.hsuniversityprograms.org/centers/former/mirees/

Moe, N. B., Aurum, A., \& Dyba, T. (2012). Challenges of shared decision-making: A multiple case study of agile software development. Information and Software Technology, 54, 853-865. doi:10.1016/j.infsof.2011.11.006

Moniz, E. J. (2012) Stimulating energy technology innovation. Doedalus, 141, 81-93. doi:10.1162/DAED_a_00148

MSC (2017). Maritime security center. Retrieved from https://www.hsuniversityprograms.org/centers/msc-maritime-security/

Moteff, J. D. (1998). Defense research: A primer on the department of defense's research, development, test and evaluation (RDT\&E) program. Congressional 
Research Service Report for Congress.

Muethel, M., \& Hoegl, M. (2013). Shared leadership effectiveness in independent professional teams. European Management Journal, 31, 423-432. doi:10.1016/j.emj.2012.11.008

Murase, T., Carter, D. R., DeChurch, L. A., \& Marks, M. A. (2014) Mind the gap: The role of leadership in multiteam system collective cognition. The Leadership Quarterly, 25, 972-986 doi:10.1016/j.leaqua.2014.06.003

National Science Foundation (2018). Science and technology centers (STCs). Retrieved from https://www.nsf.gov/od/oia/programs/stc/

NCBSI (2017). National center for border security and immigration. Retrieved from https://www.hsuniversityprograms.org/centers/former/ncbsi/

Niemann, H., Moehrle, M. G., \& Frischkorn, J. (2017). Use of a new patent text-mining and visualization method for identifying patenting patterns over time: Concept, method and test application. Technological Forecasting and Social Change, 115, 210-220. doi.org/10.1016/j.techfore.2016.10.004

Northouse, P. G. (2016). Leadership theory and practice (7th ed.). Thousand Oaks, CA: Sage.

NTSCOE (2017). National transportation security center of excellence. Retrieved from https://www.hsuniversityprograms.org/centers/former/ntscoe/

Obolensky, N. (2014). Complex adaptive leadership: Embracing paradox and uncertainty ( $2^{\text {nd }}$ ed.). Burlington, VT: Gower Publishing Company.

Ocker, R. J. \& Hiltz, S. R. (2012). Learning to work in partially distributed teams: The 
impact of team interactions on learning outcomes. Paper presented at the 45 th Hawaii International Conference on Systems Sciences, Manoa, HI. doi:10.1109/HICSS.2012.412

Oh, S. H. (2012). Leadership emergence in autonomous work teams: Who is more willing to lead? Social Behavioral and Personality, 40, 1451-1464. doi:10.2224/sbp.2012.40.9.1451

Olson, G. M., \& Olson, J. S. (2000). Distance Matters. Human-Computer Interaction, 15(2-3), 139-178. doi:10.1207/S15327051HCI1523_4

PACER (2017). Center of excellence for study of preparedness and catastrophic event response. Retrieved from https://www.hsuniversityprograms.org/centers/former/pacer/

Pacific Northwest National Laboratory. (2015) Common operating and response environment (CORE). Retrieved from http://readthis.pnl.gov/marketsource/readthis/B3051_not_print_quality.pdf

Patton, M. Q. (2015). Qualitative research and methods: Integrating theory and practice ( $5^{\text {th }}$ ed.). Thousand Oaks, CA: Sage.

Paulsen, N., Callan, V. J., Ayoko, O. \& Saunders, D. (2013). Transformational leadership and innovation in an R\&D organization experiencing major change. Journal of Organizational Change Management, 26, 595-610. doi:10.1108/09534811311328597

Perovšek, M., Kranjc, J., Erjavec, T., Cestnik, B., \& Lavrač, N. (2016). TextFlows: A visual programming platform for text mining and natural language processing. 
Science of Computer Programming, 121, 128-152.

doi:0.1016/j.scico.2016.01.001

Pinar, T., Zehir, C., Kitapci, H., \& Tanriverdi, H. (2014) The relationship between leadership behaviors team learning and performance among the virtual teams. International Business Research, 7, 68-79. doi:10.5539/ibr.v7n5p68.

Poirier, E., Forgues, D. \& Staub-French, S. (2016). Collaboration through innovation: implications for expertise in the AEC sector. Construction Management and Economics, 34 (11), 769-789. doi:10.1080/01446193.2016.1206660

Potel, M., \& P. C. Wong. (2014). Visualizing 20 years of applications. IEEE Computer Graphics and Applications, 34, 6-11. doi:0272-1716/14/\$31.00

Project Management Institute. (2013). A guide to the project management body of knowledge $\left(5^{\text {th }}\right.$ ed.). Newtown Square, PA: Author.

Ramos, A. L., Vasconcelos, J., \& Barcelo, J. (2013). LITHE: An agile methodology for human-centric model-based systems engineering. IEEE Transactions on Systems, Man, and Cybernetics Systems, 43, 504-521. doi:10.1109/TSMCA.2012.2207888

Qu, R., Janssen, O., \& Shi, K. (2015). Transformational leadership and follower creativity: The mediating role of follower relational identification and the moderating role of leader creativity expectations. The Leadership Quarterly, 26(2), 286-299. doi:10.1016/j.leaqua.2014.12.004

Sahay, K. \& Baul, U. (2015) Comparison of traditional leadership and e-leadership: A study of organizational effectiveness in today's scenario. Purushartha: A Journal of Management Ethics and Spirituality, 7, 40-59. Retrieved from 
https://www.researchgate.net/profile/Kasturi_Sahay/publication/291056715_Com parison_of_Traditional_Leadership_and_E-

Leadership_A_Study_of_Organizational_Effectiveness_in_Today\%27s_Scenario/ links/569dcac008aed27a702fdda2/Comparison-of-Traditional-Leadership-and-ELeadership-A-Study-of-Organizational-Effectiveness-in-Todays-Scenario.pdf Sanberg, P. R., Gharib, M., Harker, P. T., Kaler, E. W., Marchase, R. B., Sands, T. D., . . .\& Sarkar, S. (2014). Changing the academic culture: Valuing patents and commercialization toward tenure and career advancement. Proceedings of the National Academy of Sciences, 111, 6542-6547. doi:10.1073/pnas.1404094111

Santos, J. P., Caetano, A., \& Tavares, S. M. (2015). Is training leaders in functional leadership a useful tool for improving the performance of leadership functions and team effectiveness? The Leadership Quarterly, 26(3), 470-484. doi:10.1016/j.leaqua.2015.02.010

Scheffer, M., Bacompte, J., Bjordam, T. K., Carpenter, S. R., Clarke, L. B., Folke, C., .. . \& Westley, F. R. (2015). Dual thinking for scientists. Ecology and Society, 20(2), 3. doi:10.5751/ES-07434-200203

Schermuly, C. C., Meyer, B., \& Dämmer, L. (2015). Leader-member exchange and innovative behavior. Journal of Personnel Psychology, 12, 132-142. doi:10.1027/1866-5888/a000093

Schiller, S. Z., Mennecke, B. E., Nah, F. F. H., \& Luse, A. (2014). Institutional boundaries and trust of virtual teams in collaborative design: An experimental study in a virtual world environment. Computers in Human Behavior, 35, 565- 
577. doi:10.1016/j.chb.2014.02.051

Schilke, O., \& Cook, K. S. (2013). A cross-level process theory of trust development in interorganizational relationships. Strategic Organization, 11, 281-303. doi: $10.1177 / 1476127012472096$

Seemiller, C., \& Murray, T. (2013). The common language of leadership. Journal of Leadership Studies, 7, 33-45. doi:10.1002/j1s.21277

Seidman, I. (2013). Interviewing as qualitative research (4th ed.). New York, NY: Teachers College Press.

Serban, A., Yammarino, F. J., Dionne, S. D., Kahai, S. S., Hao, C., McHugh, K. A., .. . \& Peterson, D. R. (2015). Leadership emergence in face-to-face and virtual teams: A multi-level model with agent-based simulations, quasi-experimental and experimental tests. The Leadership Quarterly, 26, 402-418. doi:10.1016/j.leaqua.2015.02.006

Shao, J., \& Muller, R. (2011). The development of constructs of program context and program success: A qualitative study. International Journal of Project Management, 29, 947-959. doi:10.1016/j.ijproman.2011.02.003

Shrum, W., Genuth, J., \& Chompalov, I. (2007). Structures of scientific collaboration. Cambridge, MA: MIT Press.

Sidhu, J. S., \& Volberda, H. W. (2011). Coordination of globally distributed teams: A coevolution perspective on offshoring. International Business Review, 20, 278-290. doi:10.1016/j.ibusrev.2011.01.006

Stake, R. E. (1995). The art of case study research. Los Angeles, CA: Sage. 
Stake, R. E. (2006). Multiple case study analysis. New York, NY: Guilford Press.

START (2017). National consortium for the study of terrorism and responses to terrorism. Retrieved from https://www.hsuniversityprograms.org/centers/startterrorism-studies/

Su, X. (2014). Academic scientists' affiliation with university research centers: Selection dynamics. Research Policy, 43, 382-390. doi:10.1016/j.respol.2013.11.001

Trist, E. L., Higgen, G. W., Murry, H., \& Pollok A. B. (1963). Organizational choice. New York, NY: Routledge.

Turkulainen, V., Ruuska, I., Brady, T., \& Artto, K. (2015). Managing project-to-project and project-to-organization interfaces in programs: Organizational integration in a global operations expansion program. International Journal of Project Management, 33, 816-827. doi:10.1016/j.ijproman.2014.10.008

Uhl-Bien, M., Riggio, R. E., Lowe, K. B., \& Carsten, M. K. (2014). Followership theory: A review and research agenda. The Leadership Quarterly, 25(1), 83-104. doi:10.1016/j.leaqua.2013.11.007

U. S. Department of Energy. (2015). Energy Department's energy innovation hubs. Retrieved from http://energy.gov/science-innovation/innovation/hubs U. S. Department of Homeland Security (2017). Department of Homeland Security Science and Technology Centers of Excellence. Retrieved from https://www.hsuniversityprograms.org/centers/

Vessey, W. B., Barrett, J. D., Mumford, M. D., Johnson, G., \& Litwiller, B. (2014). Leadership of highly creative people in highly creative fields: A historiometric 
study of scientific leaders. The Leadership Quarterly, 25, 672-691. doi:10.1016/j.leaqua.2014.03.001

Wadsworth, M. B. \& Blanchard, A. L. (2015). Influence tactics in virtual teams. Computers in Human Behavior, 44, 386-393. doi:10.1016/j.chb.2014.11.026

Wageman, R., \& Fisher, C. (2014). Who's in charge here? The team leadership implications of authority structure. In V. D. Day (Ed.), The Oxford handbook of leadership and organizations (pp. 455-481). New York, NY: Oxford University Press.

Walsh, J. P., \& Huang, H. (2014). Local context, academic entrepreneurship and open science: Publication secrecy and commercial activity among Japanese and US scientists. Research Policy, 43, 245-260. doi:10.1016/j.respol.2013.10.003

Wang, Y. Q., Hu, N. L., \& Li, L. P. (2013). Study on technology innovation process integration based on technological growth curve. Applied Mechanics and Materials, 273, 13-17. doi:10.4028/www.scientific.net/AMM.273.13

Wiedemann, G. (2013). Opening up to big data: Computer-assisted analysis of textual data in social sciences. Historical Social Research/Historische Sozialforschung, 332-357. Retrieved from http://www.jstor.org/stable/24142701

Wiedemann, G. (2016) Text mining for qualitative data analysis in the social sciences: A study on democratic discourse in Germany. Fachmedien, Wiesbaden: Springer. doi: $10.1007 / 9783658153090$

Yin, R. K. (2014). Case study research design and methods (5th ed.). Los Angeles, CA: Sage. 
ZADD (2017). Center of Excellence for Zoonotic and Animal Disease Defense.

Retrieved from https://www.hsuniversityprograms.org/centers/emeritus/zaddanimal-disease-defense/ 
Appendix A: Telephone Interview Protocol

INTRODUCTION: Hello is this [insert interviewee's name]? My name is Gariann Gelston, and I am calling to conduct our interview regarding the Center of Excellent program study. Is this still a good time for you to speak with me?

As noted in the material that I sent earlier, I would like to record our discussion using the Microsoft Lync recorder. Are you still agreeable to recording? The Microsoft system will announce when the recording has begun. Please let me know when you hear the recording announcement. [Start recording]

I am conducting this study as partial fulfillment of the requirements for the degree of Doctor of Philosophy in Decision Science and Applied Mathematics, with a specialization in Leadership and Organizational Change. I will incorporate the results of your input into my dissertation on the follower perspective of the virtual leadership of complex multiorganizational research and development programs. This study may be useful in supporting identifying leadership strategies and constructs that could potentially be incorporated into scientific leadership training, or used in selection criteria for program leaders.

Your participation in this study will provide insights into virtual leadership strategies in complex interdisciplinary scientific collaborations. Thank you in advance for your help!

This interview will take approximately 45 minutes to complete once we complete the demographics questions. We will be finished within an hour unless you have more to share. If so, we can extend the conversation, or schedule a followup discussion. All information you provide will remain strictly confidential. At no time will your responses be associated with your personal identity. I will be reporting this information as a case study, within an aggregate of four case studies.

Are you ready to begin? 


\section{PART 1: Demographics}

I would like to begin by learning about you and your particular role in the Centers of Excellence program.

1. What is your primary discipline?

2. What is the highest degree you have achieved?

3. Approximately how many years have you been a researcher?

4. How many years have you participated in Center research?

5. Please describe the Center you participate in and how it is structured.

6. Can you describe your role in the Center and which other roles, or individuals, with whom you work most closely in the Center?

\section{PART 2: Leadership}

Now I would like to learn more about the leadership in the Center. Leadership research trends the last decade indicated a growing interest in leadership and the interest in identifying theories to capture observations. For this research, I am taking a follower perspective to learn more about leadership strategies as viewed by their program members.

7. Could you describe the leadership structure in which you operate in the Center and can you describe your role in that structure for me?

Probing points:

- Are the leaders collocated?

- Are the leaders from the same organization as the participant?

- Are the leaders from the same discipline as the participant?

8. What leader role(s) would you identify as severing you and your team? Probing points:

- Are the leaders collocated?

- If so, is there a noncollocated leader that you interact with and what does that interaction look like?

- Are the leaders from the same organization as the participant?

- Are the leaders from the same discipline as the participant?

9. How do you typically interact with that leader?

Probing points: 
- How frequently do you interact?

- What mode of interaction is most common or unusual?

- What is the level of formality in the interaction?

10. If you were the leader what strategies, or improvements, would you recommend?

11. What do you think are the advantages of not having a leader collocated?

Probing points:

- Are the leaders collocated?

- Are the leaders from the same organization as the participant?

- Are the leaders from the same discipline as the participant?

12. What disadvantages of not having your leader collocated?

Probing points:

- Are the leaders collocated?

- Are the leaders from the same organization as the participant?

- Are the leaders from the same discipline as the participant?

\section{PART 3: Motivation}

The next questions address your perspectives on the motivation for innovative research. In your role, think about the kinds of activities, incentives, or behaviors that bring out the creative interdisciplinary teamwork and new ideas.

13. What do you consider to be motivation regarding your research on a collaborative team?

14. Can you describe a situation, or scenario, where you were highly motivated?

15. What other considerations, or factors, do you think would be meaningful in understanding motivation?

16. Do you feel like this structure impacts your motivation?

Probing points:

- If so, in what ways do you field the structure impacts your motivation?

- If not, why do you think that the structure does not have an impact on motivation?

\section{PART 3: Conclusion}

Finally, I'd like to give you an opportunity to share anything that comes to mind regarding working in the Center that you think would be helpful in understanding the role of leadership and/or motivation of members. 
17. Do you have any other comments, questions, or clarifications regarding our discussion?

18. If I have additional questions would it be alright if I contact you? 
Appendix B: Initial and Final Code List Summaries

Description of coding approach and provisional code lists were used. Additional codes were added and unused codes were removed. Below is the initial code list and the final code list derived from the analyses of participant input.

\section{Provisional Code Lists}

Deductive coding was used as a provisional code list for this research based on my literature investigation. Below is an outline of the provisional code list.

- Descriptive codes - used for indexing and categorization
- Advanced degrees/education
- Collaboration
- Communication
- Competition
- Confidence
- Cooperation
- Coordination
- Creativity
- Electronic Systems
- Encouragement
- Fear
- Flexibility
- Freedom
$\circ$ Funding
- Hierarchy
- Intellectual leadership
- Interdisciplinary
- Network
- Organizational structure
- Patents/co-patents
- Prestige
- Productivity
- Publishing/publications
- Research experience
- Respect
- Teamwork
- Technology
- Time 
○ Trust

○ Virtual

- Process codes - used to describe a process or condition

- Analyzing information

- Capturing information

- Common goal/vision

- Crossing organizational boundaries

- Designing the team

- Encouraging leadership in others

- Executing tasks

- Fostering creativity

- Guiding overall direction

- Interdisciplinary collaborating

○ Managing others

○ Monitoring others

○ Organizing structure

- Providing customer-driven solutions

- Respecting member competencies

○ Sharing information

- Sharing responsibility

- Situation clarification

- Strategy clarification

- Using information

A single interview transcript segment may have several codes used to highlight a variety of analysis viewpoints. In addition to descriptive and process codes, codes used to add depth to the exploration of information will be used. Following is a provisional code list for value, emotion, magnitude, and attribute coding.

- Values Coding - capturing participants perspectives

○ (A) Attitude

$\circ$ (B) Belief

○ (V) Value

- Emotion Coding - capturing opinions

○ Appreciated

○ Concerned

- Confident

○ Frustrated

- Neglected 
- Magnitude coding - Magnitude coding will be used to identify intensity, frequency, and direction content. The number of + or - will indicate intensity.

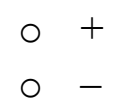

- Attribute coding - Attributes will be used for this multiple participant, multiple site, and cross-case studies study to enhance the possibility of identifying cross case patterns.

- Frequency of interactions of program members

- Number of teams within the program

- Number of participants within the program

- Number of organizations within the program

- Number of years the program has been in place

o organization type,

- University

- National laboratory

- Industry

- Government

Inductive coding was also used for additional concepts emerge during the data collection and analysis phase of this research. The overall code list will be revised as the codes are modified, deleted, or expanded to include new codes.

\section{Final Code Lists}

Final descriptive codes - used for indexing and categorization

- Unused Codes

- Complex

- Confidence

- Encouragement

- Fear

- Network

- Teamwork

- Used Provisional Codes

- Competition

- Multidisciplinary

- Technical Prestige

- Organizational Structure

- Communication

- Funding 
- Advanced degrees/education

- Coordination

- Hierarchial Structure

- Virtual

- Collaboration

- Creativity

- Intellectual Leadership

- Research Experience

- Respect

- Trust

- Flexible/Fluid

- Publishing/publications

- New Codes

- Scientific Impact

- Silos

- Politics

- Leadership Charisma

- Self Motivation

- Research Agreement

- Junior Leadership

- New Ideas

Final process codes - used to describe a process or condition

○ Unused Codes

- Interdisciplinary collaborating

- Sharing information

- Analyzing information

- Capturing information

- Crossing organizational boundaries

- Situation clarification

- Strategy clarification

- Using information

- Executing tasks

- Providing customer-driven solutions

- Used Provisional Codes

- Designing the team

- Common goal/vision

- Encouraging leadership in others 
- Fostering creativity

- Guiding overall direction

- Managing others

- Monitoring others

- Organizing structure

- Respecting member competencies

- Sharing responsibility

- New Codes

- Communication in delerivery

- Seperation from customer

- Communication in proposal

- Enjoy the research content

- Time to focus on research

- Enjoy the research process

- Administrative support important

- Weekly communication with work team

- Building Researcher friendships

- Listening to new ideas

- Academic rigor

- Leader setting direction 